\title{
Localization Expansions. I. Functions of the "Background" Configurations
}

\author{
Tadeusz Balaban \\ Department of Mathematics, Rutgers University, New Brunswick, NJ 08903, USA
}

Received: 17 July 1995/Accepted: 2 January 1996

\begin{abstract}
Expansions of the type described in the inductive hypothesis (H.5) in the paper [1] are constructed for local functions of the "background" configurations, i.e., solutions of the variational problems studied in the previous paper [3]. A main part of this construction is a further analysis of a local structure of the solutions.
\end{abstract}

\section{Introduction}

In this paper we discuss a new kind of problem connected with expansions assumed in the inductive hypothesis (H.5) in [1], but methodologically it is a continuation of the paper [3] on the variational problems for background configurations. Our main concern here is to "localize" properly these configurations, and to do this we use extensively the results and methods of [3]. One of the most important technical problems in the renormalization group approach is to construct expansions of non-local functions of basic variables into sums of localized functions, like the expansions in (H.5) [1]. There are several types of non-localities and non-local functions, we have to consider. In this paper we construct such expansions for a simplest and most frequently occurring type of non-local functions given by compositions of localized functions with one of the background configurations, like for example terms $\mathscr{E}^{(j)}\left(X ; \psi_{k}^{(j)}\right)$ of the effective actions. Obviously values $\phi_{k}(x ; \psi), \psi^{(j)}(y ; \psi)$ at points $x, y$ of corresponding lattices are non-local functions of $\psi$, in fact they depend on $\psi$ on the whole lattice, or on a generating set $\mathbb{B}_{k}$ determining the functions.

Let us describe now in detail basic goals of this paper. Consider a generating set $\mathbb{B}_{k}$, which we can identify with the sequence of domains $\left\{\Omega_{1}, \ldots, \Omega_{k}\right\}$, see the definitions (1.1)-(1.3) in [3], and assume that a next domain $\Omega_{k+1}$ is given, such that adding it to the sequence yields a new generating set $\mathbb{B}_{k+1}$. We denote $\Lambda=B\left(\Lambda_{k+1}\right)=\Omega_{k+1}^{(k)}$, i.e., $\Lambda \subset T^{(k)}$. Taking the first $j$ domains in the sequence determines a generating set $\mathbb{B}_{j}$. Consider a function $\mathscr{E}\left(X ; \psi_{j}\right)$, where $X$ is a localization domain, $X \in \mathscr{D}_{j}$, and $\psi_{j}$ is a spin variable on the lattice $T^{(j)}$. We assume that this function has the same properties as a term of the localization expansion in 
(H.5) [1], and for simplicity we assume that $X \subset \Omega_{k+1}$. After $k$ steps the variable $\psi_{j}$ is replaced by the function $\psi\left(\mathbb{B}_{j}, \mathbb{B}_{k} ; \psi_{k}\right)$, and in the $k+1^{\text {st }}$ step $\psi_{k}$ is split into the background field $\psi\left(\mathbb{B}_{k}, \mathbb{B}_{k+1} ; \theta\right)$ associated with the new spin configuration $\theta$, and the linear function $\beta_{k}^{-\frac{1}{2}} C_{\Lambda}^{(k) \frac{1}{2}} \psi$ of the ultralocal spin fluctuation variable $\psi$ that is about to be integrated out. In this situation we consider the function

$$
\mathscr{E}\left(X ; \psi\left(\mathbb{B}_{j}, \mathbb{B}_{k} ; \psi\left(\mathbb{B}_{k}, \mathbb{B}_{k+1} ; \theta\right)+\beta_{k}^{-\frac{1}{2}} C_{\Lambda}^{(k) \frac{1}{2}} \psi\right)\right),
$$

where $\theta$ is a spin configuration defined on $\mathbb{B}_{k+1}, \psi$ is the so-called spin fluctuation variable defined on $\Lambda$, and $C_{\Lambda}^{(k) \frac{1}{2}}$ is a positive square root of a positive operator $C_{\Lambda}^{(k)}$, which is an operator of a quadratic form connected with a fluctuation integral on $\Lambda$. We will write an explicit formula for this operator and we will analyze it in Sect. 3. Now let us mention only that a kernel of this operator is defined on $\Lambda \times \Lambda$, and is an analytic function of the configurations $\phi\left(\mathbb{B}_{k+1} ; \theta\right), \alpha\left(\mathbb{B}_{k+1} ; \theta\right)$. Functions of the form (1.1) appear naturally in a fluctuation integral, which will be discussed in the next paper. Obviously (1.1) is a non-local function of the variables $\theta, \psi$, and the main goal of this paper is to construct its localization expansion in both of the variables. To explain a form of this expansion we need some new definitions. We will use systematically domains in the continuous space $T$ of a type described in the definition

$$
\begin{aligned}
\mathscr{D}\left(\mathbb{B}_{j}\right)=\{ & Y: Y \subset T, Y \text { is a connected domain, connected components of } \\
& Y \cap \Omega_{n} \cap \Omega_{n+1}^{c} \text { belong to } \mathscr{D}_{n}, n \leqq j, \\
& \text { where we put here } \left.\Omega_{0}=T, \Omega_{j+1}=\emptyset\right\} .
\end{aligned}
$$

This definition holds for any $j \leqq k+1$. For such domains we generalize the concept of the linear size function $d_{j}$ introduced in (2.27) [1], and we define

$$
\begin{gathered}
\tilde{d}_{j}(Y)=\inf \left\{\frac{1}{M}|\Gamma|_{\mathbb{B}_{j}}: \Gamma \text { is a continuous tree graph contained in } Y\right. \\
\text { and intersecting all cubes } \square^{\prime} \text { contained in one of the domains } \\
\left.Y \cap \Omega_{n} \cap \Omega_{n+1}^{c} \text { and belonging to the cover } \pi_{n}^{\prime}, n \leqq j\right\}, \\
\text { where }|\Gamma|_{\mathbb{B}_{j}}=\sum_{n=1}^{j}\left(L^{n} \xi\right)^{-1}\left|\Gamma \cap \Omega_{n} \cap \Omega_{n+1}^{c}\right|_{\xi} .
\end{gathered}
$$

The length in the last equality is in the scale $\xi=L^{-j}$, which means that we take the original lattice as $T_{\xi}$, and $T^{(j)}$ as the unit lattice $T_{1}^{(j)}$.

To simplify the formula below let us denote the function $\psi\left(\mathbb{B}_{j}, \mathbb{B}_{k}\right)$ by $\psi_{k}^{(j)}$, and $\psi\left(\mathbb{B}_{k}, \mathbb{B}_{k+1}\right)$ by $\psi^{(k)}$. The main goal of this paper is to prove the following statement:

The function (1.1) has a localization expansion of the form

$$
\mathscr{E}\left(X ; \psi_{k}^{(j)}\left(\psi^{(k)}(\theta)+\beta_{k}^{-\frac{1}{2}} C_{\Lambda}^{(k) \frac{1}{2}} \psi\right)\right)=\sum_{Y \in \mathscr{D}\left(\mathbb{B}_{k+1}\right): Y \supset X} \mathscr{E}(X, Y ; \theta, \psi),
$$

where the terms of the expansion depend on the variables $\theta, \psi$ restricted to the corresponding domains $Y$ and satisfy the bounds

$$
|\mathscr{E}(X, Y ; \theta, \psi)|<E_{0} \exp \left(-\kappa_{0} d_{j}(X)-2 \kappa \tilde{d}_{k+1}(Y)\right) .
$$


The formula (1.4) has prepared for integrating out $\psi$ in such a way that localization in $\theta$ will be manifest afterwards, because $\psi$ is an ultralocal variable. The formulation of the above statement is not complete yet because we have not described analyticity domains for the terms of the expansion, but we postpone formulations and a discussion of these more technical issues to Sect. 4. The statement gives a precise formulation of main aspects of these localization expansions, and it establishes a pattern for all related propositions in this and subsequent papers.

Let us explain briefly what is a main problem connected with constructing the expansion (1.4). It should be quite clear that it has to do with appropriate localization expansions of the functions $\psi_{k}^{(j)}, \psi^{(k)}, C_{\Lambda}^{(k) \frac{1}{2}}$, because they introduce a non-locality into the function (1.1). However, it is not enough to localize them in an arbitrary way, the point is that we have to use representations and bounds of Sect. 3 [1], in particular Corollary 3.11 [1], and they hold on rather small subspaces of natural analyticity domains, like subspaces $\Psi_{j}^{c}\left(X ; C_{0} L^{j} \eta, \varepsilon_{k}\right)$ defined in (3.13), (3.14) [1]. These subspaces incorporate in their definitions various scaling properties, some of them "anomalous," related to the singular nature of the basic variational equations for the problem (1.6) [3]. We have to localize the function $\psi_{k}^{(j)}$ in such a way that the properties are preserved at least on the domain $X$, so its values are in the subspace above. This problem is solved in two steps, actually in three, the first step was the introduction of the auxiliary variable $\alpha$ and the variational equations (1.10) [3], in which the parameter $\lambda$ occurs only as $\frac{1}{\lambda}$. The important step is to use the fundamental composition formula (4.39) [3] together with the representation (4.21) [3], and to write $\psi_{k}^{(j)}$ as a composition of such a function localized in a neighbourhood of $X$, and a suitable non-local function, which enters only into "boundary conditions" of the local one, and may satisfy more relaxed conditions described by corresponding spaces $\widetilde{\Psi}^{c}$ in $(1.11),(1.12)$ [3]. Such a representation of the function $\psi_{k}^{(j)}$ is presented in the formula (4.16), which is one of the most important formulas in the paper, a key to the localization procedure. It reduces the problem to a simpler one of localizing the functions of the type $\psi\left(\mathbb{B}_{j}, \mathbb{B}_{k}^{\prime}\right)$ with fewer and simpler conditions to be satisfied. This is reduced again, through the representation (4.21) [3], to the problem of localizing the function $\phi\left(\mathbb{B}_{k}\right)$, which is still not quite standard, in particular the second non-linear variational equation in (1.14) [3] has to be treated with some care, and this is the third of the steps mentioned above, but it is resolved in a number of small technical details not worthwhile, or even impossible to describe here.

Let us describe now the content of the following three sections. We reverse in a sense the steps described above, and we start with localizations of the functions $\phi\left(\mathbb{B}_{k}\right), \psi\left(\mathbb{B}_{j}, \mathbb{B}_{k}\right)$, which is the subject of Sect. 2. It is achieved by introducing suitable interpolating parameters into their definitions and studying analytic extensions in the parameters, their regularity properties and bounds. We do the same for the covariance operator $C_{\Lambda}^{(k)}$, and for some functions of this operator, in Sect. 3. In Sect. 4 we start with a derivation of the representation (4.16), and then we construct the expansion (1.4) using the results of the previous sections and applying some simple interpolation formulas. All results of this paper are very technical and it is more convenient to start with definitions and constructions, and then to formulate the most important conclusions as propositions; for some of them it is even not possible to do it another way.

Finally let us remark that the size $M$ of large cubes in the following two sections does not have to be equal to the size of large cubes used in the renormalization 
group procedure. In these sections we consider $M=L^{m}$ as a parameter which must satisfy appropriate conditions, in particular $M^{-1}$ is one of the fundamental factors controlling convergence of all expansions we will construct in this paper. These issues will be discussed in the last section.

\section{Localization Expansions for the "Background" Configurations}

In this section we introduce the localization parameters into the functions $\phi\left(\mathbb{B}_{k}\right)$, $\psi\left(\mathbb{B}_{j}, \mathbb{B}_{k}^{\prime}\right)$, and other functions we use in these papers. This construction for functions $\phi\left(\mathbb{B}_{k}\right)$ is a basis of all other constructions, so we start with its detailed description. We use here extensively the constructions and the results of the papers $[2,3]$.

We fix now a generating set $\mathbb{B}_{k}$, and we denote the functions $\phi\left(\mathbb{B}_{k}\right), \alpha\left(\mathbb{B}_{k}\right)$ simply by $\phi_{k}, \alpha_{k}$. Our basic goal is to construct extensions of the functions $\phi_{k}, \alpha_{k}$ in the interpolating parameters, and to investigate their analyticity properties and bounds. This is based on the proof of Proposition 3.1 given in the third section of [3]. We start with the representation $\phi_{k}=\phi_{0}+\delta \phi_{k}, \alpha_{k}=\alpha_{0}+\delta \alpha_{k}$. The functions $\phi_{0}, \alpha_{0}$ are already almost local functions of $\psi+\psi^{\prime}, h+h^{\prime}$, as it follows from their definitions (3.2), (3.3) [3]. More precisely $\phi_{0}, \alpha_{0}$ restricted to $\Delta_{j}(y), y \in \Lambda_{j}$, depend on $\psi+\psi^{\prime}, h+h^{\prime}$ restricted to $\tilde{\Delta}_{j}(y)$. Thus the basic problem mentioned above concerns the functions $\delta \phi_{k}, \delta \alpha_{k}$. They are constructed as solutions of Eqs. (3.19), and we consider again the more general system (3.20), to cover some other applications. The function $\delta \phi_{k}$ is represented in the form (3.23), to which we apply the identity (3.25). We write explicitly this representation for future reference

$$
\begin{aligned}
\delta \phi_{k}= & G\left(\alpha_{0}\right)\left(Q^{*} a \delta \psi+f_{1}\right)-G\left(\alpha_{0}\right) \phi_{0} \delta \alpha_{k}-G\left(\alpha_{0}\right) \delta \alpha_{k} G\left(\alpha_{0}\right)\left(Q^{*} a \delta \psi+f_{1}\right) \\
& +G\left(\alpha_{0}\right) \delta \alpha_{k} G\left(\alpha_{0}\right) \phi_{0} \delta \alpha_{k}+G\left(\alpha_{0}\right) \delta \alpha_{k} G\left(\alpha_{0}\right) \delta \alpha_{k} G\left(\alpha_{0}+\delta \alpha_{k}\right)\left(Q^{*} a \delta \psi+f_{1}\right) \\
& -G\left(\alpha_{0}\right) \delta \alpha_{k} G\left(\alpha_{0}\right) \delta \alpha_{k} G\left(\alpha_{0}+\delta \alpha_{k}\right) \phi_{0} \delta \alpha_{k} .
\end{aligned}
$$

The function $\delta \alpha_{k}$ satisfies Eqs. (3.24), or (3.26). We consider at first the function $\delta \phi_{k}$, or a more general function given by the above formula with $\delta \alpha_{k}$ replaced by some $\delta \alpha$. We introduce the interpolating parameters based on the generalized random walk expansion for $G(\alpha)$ constructed in Sect. A of [2b], see in particular the formula (2.40). Let us describe this expansion. We start with a construction of a cover of the domain $\Omega_{1}$. Each $\Omega_{j}$ is a localization domain from $\mathscr{D}_{j}$, so it is a union of large cubes in $L^{-j}$-scale, or a union of $M L^{j} \eta$-cubes in the $\eta$-scale, with centers at points of the lattice $T_{M L^{j} \eta}^{(j+m)}$, where $M=L^{m}$. We take the domain $\Omega_{j}^{\sim}$, i.e. the domain obtained by adding one layer of the large cubes touching $\Omega_{j}$, and the set

$$
\Lambda_{j}^{(m)+}=\left(\Omega_{j}^{\sim} \backslash \Omega_{j+1}\right) \cap T_{M L^{j} \eta}^{(j+m)},
$$

which is the set of centers of the large cubes contained in $\Omega_{j}^{\sim}$ and disjoint with $\Omega_{j+1}$. For each point $z$ in this set we take a cube of the size $3 M L^{j} \eta$ with a center at $z$ and denote it by $\square_{z}$. The family of cubes

$$
\left\{\square_{z}: z \in \bigcup_{j=1}^{k} \Lambda_{j}^{(m)+}\right\} \text { is a cover of the domain } \Omega_{1} \text {. }
$$


We take a decomposition of unity corresponding to this cover and similar to the decomposition in (3.1) [3]. More precisely we take a decomposition of unity $\left\{h_{z}\right\}$ with functions $h_{z}$ having the properties

$$
\begin{gathered}
h_{z} \in C_{0}^{2}\left(\square_{z}\right), \quad h_{z} \geqq 0, \quad\left|\partial h_{z}\right|<2\left(M L^{j} \eta\right)^{-1}, \quad\left|\partial \partial h_{z}\right|<4\left(M L^{j} \eta\right)^{-2}, \\
\sum_{z} h_{z}^{2}=1 \quad \text { on a neighborhood of } \Omega_{1} .
\end{gathered}
$$

It is easy to construct such a decomposition starting with a function $h \in C_{0}^{2}(]-1$, 1[)$, 0 \leqq h \leqq 1,\left|h^{\prime}\right|<2,\left|h^{\prime \prime}\right|<4$, and such that $h^{2}(t)+h^{2}(t-1)=1$ on a neighborhood of the interval $[0,1]$. Then almost all functions $h_{z}$ can be defined by the formula $h_{z}(x)=\prod_{\mu=1}^{d} h\left(\left(M L^{j} \eta\right)^{-1}\left(x_{\mu}-z_{\mu}\right)\right)$, that is for all $z$ except those in the boundary layers $\Omega_{j}^{\sim} \backslash \Omega_{j}$, where two adjacent scales meet. It is easy to see how to adjust the definition for those points, so we do not write the rather awkward formulas here.

For each cube $\square_{z}$ of the cover we take the basic operator $-\Delta_{\Omega_{1}}^{D, \eta}+Q^{*} a Q+v+\alpha$ and restrict it to $\square_{z}$ taking the Neumann boundary conditions on the part of the boundary contained in $\Omega_{1}$. Denoting the corresponding Laplace operator by $\left(\Delta_{\Omega_{1}}^{D, \eta}\right)_{\square_{z}}^{N}$ we define

$$
G\left(\square_{z}, \alpha\right)=\left(-\left(\Delta_{\Omega_{1}}^{D, \eta}\right)_{\square_{z}}^{N}+\left(Q^{*} a Q+v+\alpha\right)\left\lceil_{\square_{z} \cap \Omega_{1}}\right)^{-1} .\right.
$$

For all $\square_{z}$ which do not intersect the boundary $\partial \Omega_{1}$ the Laplace operator is simply $\Delta_{\square}^{N, \eta}$. Also the operator $Q^{*} a Q$ restricted to $\square_{z}$ is either equal to an operator $a_{j}\left(L^{j} \eta\right)^{-2} Q_{j}^{*} Q_{j}$ if $\square_{z}$ is contained in $\Omega_{j} \backslash \Omega_{j+1}$, or to a sum of two such operators for $j$ and $j+1$, restricted to the subdomains $\square_{z} \cap \Omega_{j+1}^{c}$ and $\square_{z} \cap \Omega_{j+1}$, if $\square_{z}$ intersects the boundary $\partial \Omega_{j+1}$. The inverse operators (2.3) have been investigated thoroughly in the paper [2b], see in particular the bound (2.33) there. We reformulate this bound here, but at first we have to recall the definition of the scaled distance $d\left(y, y^{\prime}\right)$ between two points $y, y^{\prime} \in \mathbb{B}_{k}$. Take the set of all paths $\Gamma_{y, y^{\prime}}$ connecting the points $y, y^{\prime}$, contained in $\Omega_{1}$, and such that the intersections $\Gamma_{y, y^{\prime}} \cap\left(\Omega_{j} \backslash \Omega_{j+1}\right)$ are unions of paths in the lattice $\Lambda_{j}$, i.e. are unions of bonds of this lattice. Define

$$
d\left(y, y^{\prime}\right)=\inf _{\Gamma_{y, y^{\prime}}}\left|\Gamma_{y, y^{\prime}}\right|_{\mathbb{B}_{k}},|\Gamma|_{\mathbb{B}_{k}}=\sum_{j=1}^{k}\left(L^{j} \eta\right)^{-1}\left|\Gamma \cap \Omega_{j} \cap \Omega_{j+1}^{c}\right| .
$$

This function is determined by the set $\mathbb{B}_{k}$, and it is obviously a distance on this set. We will need all the properties of this function proved in [2b], in particular the bounds (2.50)-(2.53) in Lemma 2.1. Notice that if $y, y^{\prime} \in \square_{z} \subset \Omega_{j} \backslash \Omega_{j+1}$, then $d\left(y, y^{\prime}\right)=\left(L^{j} \eta\right)^{-1}\left|y-y^{\prime}\right|$, where $\left|y-y^{\prime}\right|=\left|y-y^{\prime}\right|_{1}=\sum_{\mu=1}^{d}\left|y_{\mu}-y_{\mu}^{\prime}\right|$ is the distance in the $\eta$-scale.

If $\square_{z}$ intersects the boundary $\partial \Omega_{j+1}$, then we have the bounds $\left(L^{j+1} \eta\right)^{-1} \mid y-$ $y^{\prime}\left|\leqq d\left(y, y^{\prime}\right) \leqq\left(L^{j} \eta\right)^{-1}\right| y-y^{\prime} \mid$. Now we can formulate the following property of the operators (2.3): there exist positive constants $B_{0}, \gamma_{0}, \gamma_{1}$ such that if $\alpha$ has complex values and satisfies $|\alpha|_{2}<\gamma_{1}$, the norm restricted to $\square_{z}, y \in \square_{z} \cap \Lambda_{j}, y^{\prime} \in \square_{z} \cap \Lambda_{j^{\prime}}$, then

$$
\begin{aligned}
& L^{j} \eta\left|\chi_{\Delta_{j}(y)} \partial^{\eta} G\left(\square_{z}, \alpha\right) \chi_{\Delta_{J^{\prime}}\left(y^{\prime}\right)} f\right|+\left|\chi_{\Delta_{j}(y)} G\left(\square_{z}, \alpha\right) \chi_{\Delta_{J^{\prime}}\left(y^{\prime}\right)} f\right| \\
& \leqq B_{0} e^{-\gamma_{0} d\left(y, y^{\prime}\right)}\left(L^{j^{\prime}} \eta\right)^{2}\left|\chi_{\Delta_{j^{\prime}}\left(y^{\prime}\right)} f\right|
\end{aligned}
$$


for any function $f$ defined on $\square_{z} \cap \Omega_{1}$. This is the bound (2.33) in [2b], after rescaling to the $\eta$-lattice and using the distance (2.4). Next, we define an operator $K_{z}=K\left(h_{z}\right)$ by the formula

$$
\begin{aligned}
\left(K_{z} f\right)(x)= & \sum_{b \in s t(x)}\left(\partial^{\eta} h_{z}\right)(b)\left(\partial^{\eta} f\right)(b)+\left(\Delta^{\eta} h_{z}\right)(x) f(x) \\
& -a_{j}\left(L^{j} \eta\right)^{-2} \sum_{x^{\prime} \in B_{j}(y)} L^{-j d}\left(h_{z}\left(x^{\prime}\right)-h_{z}(x)\right) f\left(x^{\prime}\right) \\
& \text { for } x \in B_{j}(y), y \in \square_{z} \cap \Lambda_{j},
\end{aligned}
$$

or in the operator form

$$
K\left(h_{z}\right)=2 \nabla h_{z} \cdot \nabla+\Delta h_{z}-\left[Q^{*} a Q, h_{z}\right] .
$$

We will use this definition also in cases in which $h_{z}$ is replaced by some other functions, possibly vector valued. Using the properties (2.2) of the functions $h_{z}$ we obtain the following simple bound for the operators $K_{z}$ :

$$
\begin{aligned}
\left|\chi_{\Delta_{j}(y)} K_{z} f\right| \leqq & 4 d\left(M L^{j} \eta\right)^{-1}\left|\chi_{\Delta_{j}(y)} \partial^{\eta} f\right|+4 d\left(M L^{j} \eta\right)^{-2}\left|\chi_{\Delta_{j}(y)} f\right| \\
& +\frac{3}{2}\left(L^{j} \eta\right)^{-2} 2 d M^{-1}\left|\chi_{\Delta_{j}(y)} f\right| \\
\leqq & 4 d M^{-1}\left(\left(L^{j} \eta\right)^{-1}\left|\chi_{\Delta_{j}(y)} \partial^{\eta} f\right|+\left(L^{j} \eta\right)^{-2}\left|\chi_{\Delta_{j}(y)} f\right|\right)
\end{aligned}
$$

for $y \in \square_{z} \cap \Lambda_{j}$.

At first we construct the generalized random walk expansion for the operator $G(\alpha)$ defined by (3.22) [3]. These random walks are sequences $\omega=\left(\omega_{0}, \omega_{1}, \ldots, \omega_{n}\right)$, $n=0,1, \ldots$ such that $\omega_{i} \in \bigcup_{j=1}^{k} \Lambda_{j}^{(m)+}$, i.e. $\omega_{i}$ are centers of cubes belonging to the considered cover, and $\square_{\omega_{i}} \cap \square_{\omega_{i+1}} \neq \emptyset$. The expansion has the following form:

$$
G(\alpha)=\sum_{\omega} h_{\omega_{0}} G\left(\square_{\omega_{0}}, \alpha\right) h_{\omega_{0}} K_{\omega_{1}} G\left(\square_{\omega_{1}}, \alpha\right) h_{\omega_{1}} \cdot \ldots \cdot K_{\omega_{n}} G\left(\square_{\omega_{n}}, \alpha\right) h_{\omega_{n}} .
$$

We have proved in [2b], see the proof of Proposition 2.2, that the series above is convergent in all the relevant norms, like the norms $|\cdot|_{0},\left|\partial^{\eta} \cdot\right|_{1},\left|\partial^{\eta} \cdot\right|_{(\alpha), 2-\alpha}$, $\left|\Delta^{\eta} \cdot\right|_{2}$, and the operator $G(\alpha)$ satisfies the bounds (2.57) in that theorem. We have used these bounds in Sect. 3 [3]. Now we need some stronger estimates for the series. Let us simplify the notation and denote the cube $\Delta_{j}(y)$ for $y \in \Lambda_{j}$ by $\Delta(y)$. We have the equality $1=\sum_{y \in \mathbb{B}_{k}} \chi_{\Delta(y)}$ on $\Omega_{1}$, and we insert these decompositions between the factors $h_{\omega_{l}}$ and $K_{\omega_{l+1}}$ in (2.8). Denoting $|\omega|=n$, where $\omega$ is a sequence $\left(\omega_{0}, \omega_{1}, \ldots, \omega_{n}\right)$, we have

$$
\begin{aligned}
\chi_{\Delta(y)} G(\alpha) f= & \sum_{n=0}^{\infty} \sum_{\omega:|\omega|=n} \sum_{\left(y_{1}, y_{2}, \ldots, y_{n}, y^{\prime}\right)} \chi_{\Delta(y)} h_{\omega_{0}} G\left(\square_{\omega_{0}}, \alpha\right) h_{\omega_{0}} \chi_{\Delta\left(y_{1}\right)} \\
& \cdot \chi_{\Delta\left(y_{1}\right)} K_{\omega_{1}} G\left(\square_{\omega_{1}}, \alpha\right) h_{\omega_{1}} \chi_{\Delta\left(y_{2}\right)} \\
& \cdot \ldots \cdot \chi_{\Delta\left(y_{n}\right)} K_{\omega_{n}} G\left(\square_{\omega_{n}}, \alpha\right) h_{\omega_{n}} \chi_{\Delta\left(y^{\prime}\right)} f .
\end{aligned}
$$

At first we estimate the number of walks $\omega$ with a fixed $|\omega|=n$. The number of $\omega_{0}$ such that $\Delta(y) \subset \square_{\omega_{0}}$ is not greater than $2 \cdot 2^{d}$, because there are at most $2^{d}$ 
cubes of a fixed scale containing $\Delta(y)$. For $\omega_{i}$ fixed there are less than $(2 L+1)^{d}$ points $\omega_{i+1}$ such that supp $h_{\omega_{i+1}} \cap \operatorname{supp} h_{\omega_{i}} \neq \emptyset$, and generally, if $\omega_{i}$ is not in one of the boundary layers, there are $3^{d}$ such points. Thus the number of walks does not exceed $2^{d+1}(2 L+1)^{n d}$. Next, consider one factor in (2.9) enclosed between two characteristic functions. From the bounds (2.7), (2.5), we get

$$
\left(L^{j_{1}} \eta\right)^{2}\left|\chi_{\Delta\left(y_{1}\right)} K_{z} G\left(\square_{z}, \alpha\right) h_{z} \chi_{\Delta\left(y_{2}\right)} f\right| \leqq 4 d B_{0} M^{-1} e^{-\gamma_{0} d\left(y_{1}, y_{2}\right)}\left(L^{j_{2}} \eta\right)^{2}\left|\chi_{\Delta\left(y_{2}\right)} f\right| .
$$

Applying this bound and (2.5) to the sum in (2.9) we obtain

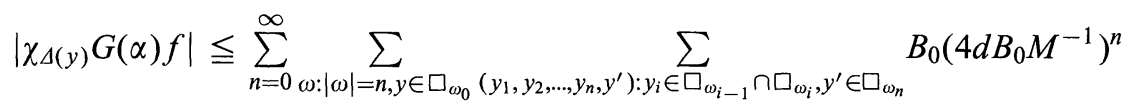

$$
\begin{aligned}
& \text { - } e^{-\gamma_{0} d\left(y, y_{1}\right)} e^{-\gamma_{0} d\left(y_{1}, y_{2}\right)} \\
& \text { - ... } e^{-\gamma_{0} d\left(y_{n-1}, y_{n}\right)} e^{-\gamma_{0} d\left(y_{n}, y^{\prime}\right)}\left(L^{j^{\prime}} \eta\right)^{2}\left|\chi_{\Delta\left(y^{\prime}\right)} f\right| \\
& \leqq \sum_{n=0}^{\infty} B_{0} c_{1}\left(d, \frac{1}{4} \gamma_{0}\right)\left(4 d B_{0} c_{1}\left(d, \frac{1}{4} \gamma_{0}\right) M^{-1}\right)^{n} \\
& \text { - } \sum_{\omega:|\omega|=n, y \in \square_{\omega_{0}}} e^{-\frac{1}{2} \gamma_{0} d(y ; \omega)} \sup _{y^{\prime} \in \square_{\omega_{n}}}\left(L^{j^{\prime}} \eta\right)^{2}\left|\chi_{\Delta\left(y^{\prime}\right)} f\right| \\
& \leqq \sum_{n=0}^{\infty} B_{0} c_{1}\left(d, \frac{1}{4} \gamma_{0}\right)\left(8 d B_{0} c_{1}\left(d, \frac{1}{4} \gamma_{0}\right)(2 L+1)^{d} M^{-1}\right)^{n} \\
& \text { - } \sum_{\omega:|\omega|=n, y \in \square_{\omega_{0}}} \frac{1}{2^{n}} \cdot \frac{1}{(2 L+1)^{d n}} \cdot e^{-\frac{1}{2} \gamma_{0} d(y ; \omega)}|f|_{2} \\
& \leqq 2^{d+2} B_{0} c_{1}\left(d, \frac{1}{4} \gamma_{0}\right)|f|_{2} \text {, where } \\
& d(y ; \omega)=\inf \left(d\left(y, y_{1}\right)+d\left(y_{1}, y_{2}\right)+\cdots+d\left(y_{m}, y^{\prime}\right)\right), \\
& \text { the infimum is taken over }\left(y_{1}, y_{2}, \ldots, y_{n}, y^{\prime}\right) \text { : } \\
& y_{i} \in \square_{\omega_{i-1}} \cap \square_{\omega_{l}}, y^{\prime} \in \square_{\omega_{n}} \text {, assuming that } y \in \square_{\omega_{0}} \text {; } \\
& c_{1}(d, \gamma)=12 c_{0}^{d}(\gamma), c_{0}(\gamma)=\sum_{x \in \mathbb{Z}} e^{-\gamma|x|} \\
& =\frac{1+e^{-\gamma}}{1-e^{-\gamma}}<\frac{4}{\gamma} \text { if } 0<\gamma \leqq 1 \text {; }
\end{aligned}
$$

and assuming $8 d B_{0} c_{1}\left(d, \frac{1}{4} \gamma_{0}\right)(2 L+1)^{d} M^{-1} \leqq 1$.

In the second inequality above we have used Lemma 2.1 in [2b]. We have written these inequalities in such detailed form because it is not the final bound which is most interesting for us, but bounds and expressions in the intermediate stages, which indicate possible improvements. In particular we have not made proper use of the factors $M^{-n}$ and $\exp \left(-\frac{1}{2} \gamma_{0} d(y ; \omega)\right)$, for example we may replace $M^{-1}$ by $M^{-\frac{1}{2}}$ in the last assumption, and use the factor $M^{-\frac{1}{2} n}$ for some other bounds. This remark will be important when we will study analytic extensions in interpolating parameters. 
Another possibility is to represent the sum in (2.9) as a sum over domains $X$ containing the cube $\Delta(y)$, with terms equal to the sum of terms in (2.9) determined by random walks $\omega$ such that $X_{\omega}=\bigcup \square_{\omega_{i}}=X$. These terms are localized in the domain $X$ in the sense that they depend on $\alpha$ and $f$ restricted to $X$, and we can use the exponential factor to get a bound of the form (H.5). This way we obtain a localization expansion for the operator $G(\alpha)$, but it is not enough for our purposes, so we do not discuss any details.

Consider the representation (2.1) of the function $\delta \phi_{k}(\delta \alpha)$ obtained by replacing the function $\delta \alpha_{k}$ by the variable $\delta \alpha$. We expand each operator $G\left(\alpha_{0}\right)$ and $G\left(\alpha_{0}+\delta \alpha\right)$ in this formula into the generalized random walk expansions (2.8). It is easy to see that multiplying and summing these expansions yield an expansion of basically the same form as (2.8), but with some small modifications. Some operators $K_{\omega_{i}}$ may be replaced by a multiplication operator, the multiplication by the function $-\delta \alpha h_{\omega_{l}}$. We can have at most two such replacements. The localized Green's operators depend on $\alpha_{0}$, except the case when there are exactly two replacements, then the operators occurring after the last one depend on $\alpha_{0}+\delta \alpha$. Finally, at the end of each term of the expansion there is a function $f$, which is equal either to $Q^{*} a \delta \psi+f_{1}$, or to $-\phi_{0} \delta \alpha$. We can represent this expansion in the form of a generalized random walk expansion, like in (2.8), if we introduce random walks $\omega=\left(\omega_{0}, \omega_{1}, \ldots, \omega_{n}\right)$ whose elements $\omega_{i}$ are not points, but pairs $\omega_{i}=\left(z_{i}, \sigma_{i}\right)$, where $z_{i} \in \bigcup_{j=1}^{k} \Lambda_{j}^{(m)+}$, $\sigma_{i} \in\{0,1\}$. We define operators $K_{\omega_{l}}$, variables $\vartheta_{i}$, and functions $g_{\sigma}, \sigma \in\{0,1\}$, as follows:

$$
\begin{aligned}
K_{\omega_{i}} & =K_{z_{i}} \quad \text { if } \sigma_{i}=0, \quad K_{\omega_{i}}=-\delta \alpha h_{z_{l}} \quad \text { if } \sigma_{i}=1 ; \\
\vartheta_{i} & =0 \quad \text { if } \sigma_{1}+\cdots+\sigma_{i}<2, \quad \vartheta_{i}=1 \quad \text { if } \sigma_{1}+\cdots+\sigma_{i} \geqq 2, i \geqq 1 ; \\
g_{0} & =Q^{*} a \delta \psi+f_{1}, \quad g_{1}=-\phi_{0} \delta \alpha .
\end{aligned}
$$

The random walks $\omega$ are restricted by the conditions: $\square_{z_{l-1}} \cap \square_{z_{l}} \neq \emptyset, i=1, \cdots, k$, $\square_{z_{l-1}}=\square_{z_{i}}$, or $z_{i-1}=z_{i}$ if $\sigma_{i}=1, \sigma_{1}+\cdots+\sigma_{k} \leqq 2$. The first condition actually has the stronger form: if both cubes $\square_{z_{l-1}}, \square_{z_{l}}$ are in the same scale, then $\square_{z_{l}} \subset \square_{z_{l-1}}^{\sim}$, hence also $\square_{z_{l-1}} \subset \square_{z_{i}}^{\sim}$, and if they are in adjacent scales, then the cube in the smaller scale is contained in the larger cube. We will use sometimes this stronger condition in the future. The expansion of the function $\delta \phi_{k}(\delta \alpha)$ can be written now in the following form:

$$
\delta \phi_{k}(\delta \alpha)=\sum_{\omega} h_{z_{0}} G\left(\square_{z_{0}}, \alpha_{0}\right) h_{z_{0}}\left(\prod_{i=1}^{|\omega|} K_{\omega_{i}} G\left(\square_{z_{l}}, \alpha_{0}+\vartheta_{i} \delta \alpha\right) h_{z_{i}}\right) g_{\sigma_{0}} .
$$

Let us remark that there is an even simpler expansion of this function, exactly of the form (2.8), if we use the representation (3.23) [3] directly, i.e. we write $\delta \phi_{k}(\delta \alpha)=G\left(\alpha_{0}+\delta \alpha\right)\left(Q^{*} a \delta \psi+f_{1}-\phi_{0} \delta \alpha\right)$, and we use the expansion (2.8) for $G\left(\alpha_{0}+\delta \alpha\right)$. This is enough for a localization of the function $\delta \phi_{k}(\delta \alpha)$, but we have to study and localize the equation for $\delta \alpha$, and for this we need the more detailed form (2.12). This expansion can be bounded as in (2.11), with some small modifications only. We will discuss this bound later, after introducing interpolating parameters. To do this we have to describe at first a geometric setting.

Consider two domains $X_{0}, X_{1}$ such that

$X_{0}, X_{1} \subset \Omega_{k}, X_{0}, X_{1} \in \mathscr{D}_{k}, X_{0}^{\sim r_{0}} \subset X_{1}, r_{0}$ is a positive integer to be fixed later . 
We could consider more general domains from $\mathscr{D}\left(\mathbb{B}_{k}\right)$, but in future applications we will use only the above simpler case. We would like to localize the function $\delta \phi_{k}(\delta \alpha)$ restricted to the domain $X_{0}$, i.e. to represent it as a sum over domains $X \supset X_{1}$ of functions which depend on $\phi_{0}, \alpha_{0}, \delta \alpha, \delta \psi, f_{1}$ restricted to $X$. We do it by introducing interpolating, or localization parameters into the formula (2.13) for this function. Let us introduce at first the notations:

$$
\begin{aligned}
& \text { for a walk } \begin{aligned}
\omega & =\left(\omega_{0}, \omega_{1}, \ldots, \omega_{n}\right) \text { we denote } \\
\tilde{\omega} & =\left(\omega_{1}, \ldots, \omega_{n}\right) \text { and }|\tilde{\omega}|=|\omega|=n \\
(K G)(\tilde{\omega}) & =\prod_{i=1}^{|\omega|} K_{\omega_{l}} G\left(\square_{z_{l}}, \alpha_{0}+\vartheta_{i} \delta \alpha\right) h_{z_{l}} \quad \text { if }|\tilde{\omega}|>0 \\
(K G)(\tilde{\omega}) & =1 \text { if }|\tilde{\omega}|=0 .
\end{aligned}
\end{aligned}
$$

The domains $\Omega_{j} \backslash \Omega_{j+1}$ are unions of disjoint large cubes in the $L^{-j}$-scale, i.e., the cubes from the partition $\pi_{j}$, where $\pi_{j}$ was defined in [1] between (2.25) and (2.26). We denote these cubes by $\square$, and to each cube we assign a variable $s_{\square}$. The set $s=\left\{s_{\square}\right\}$ is the set of interpolating, or localization parameters. We define

$$
\delta \phi_{k}(\delta \alpha, s)=\sum_{\omega} h_{z_{0}} G\left(\square_{z_{0}}, \alpha_{0}\right) h_{z_{0}}(K G)(\tilde{\omega}) g_{\sigma_{0}} \prod_{\square \subset X_{\omega} \cap X_{1}^{c}} s_{\square},
$$

where $X_{\omega}=\bigcup_{i=0}^{|\omega|} \square_{z_{l}}$. This function has the following simple properties:

$$
\begin{aligned}
\delta \phi_{k}(\delta \alpha, 1)= & \delta \phi_{k}(\delta \alpha), \text { where } s=1 \text { means that all } s_{\square}=1 ; \\
& \chi_{X_{0}} \delta \phi_{k}(\delta \alpha, 0) \text { is the sum in }(2.13) \text { over random walks } \omega \\
& \text { satisfying } \square_{z_{0}}^{\sim} \cap X_{0} \neq \emptyset, X_{\omega} \subset X_{1} \text {, hence it is localized in } X_{1} .
\end{aligned}
$$

It is obviously an analytic function of $s$, actually it is a polynomial in $s$, and we consider it on the polydisc $\left\{s:|s| \leqq e^{\kappa_{1}}\right\}$, where $\kappa_{1}$ is a sufficiently large positive number. We would like to prove bounds (3.47) in Proposition 3.1 [3] for the function (2.16), assuming that $M$ is chosen sufficiently large, depending on $\kappa_{1}$. We can obtain them in the same way as the bound (2.11), and we have for $y \in \mathbb{B}_{k}$,

$$
\begin{aligned}
\left|\chi_{\Delta(y)} \delta \phi_{k}(\delta \alpha, s)\right| \leqq B_{0} c_{1}\left(d, \frac{1}{4} \gamma_{0}\right) \sum_{\omega: \Delta(y) \subset \square_{z_{0}}} \frac{1}{2^{|\omega|}} \frac{1}{(2 L+1)^{d|\omega|}} e^{-\frac{1}{2} \gamma_{0} d(y ; \omega)} \\
\cdot\left[M^{-\frac{1}{2}|\omega|}+|\omega| M^{-\frac{1}{2}(|\omega|-1)} 2 B_{0} c_{1}\left(d, \frac{1}{4} \gamma_{0}\right)(2 L+1)^{d}|\delta \alpha|_{2} \theta_{+}(|\omega|-1)\right. \\
\left.+\frac{1}{2}|\omega|(|\omega|-1) M^{-\frac{1}{2}(|\omega|-2)}\left(2 B_{0} c_{1}\left(d, \frac{1}{4} \gamma_{0}\right)(2 L+1)^{d}|\delta \alpha|_{2}\right)^{2} \theta_{+}(|\omega|-2)\right] \\
\cdot\left(\frac{3}{2}|\delta \psi|+\left|f_{1}\right|_{2}+\frac{3}{2}|\delta \alpha|_{2}\right) \exp \kappa_{1} \sum_{j=0}^{k} \frac{1}{\left(M L^{j} \eta\right)^{d}}\left|X_{\omega} \cap X_{1}^{c} \cap \Omega_{j} \cap \Omega_{j+1}^{c}\right|,
\end{aligned}
$$

where we use the function $\theta_{+}(t)=1$ for $t \geqq 0, \theta_{+}(t)=0$ for $t<0$. 
Here we have used the first two inequalities in (2.11) together with the assumption $8 d B_{0} c_{1}\left(d, \frac{1}{4} \gamma_{0}\right)(2 L+1)^{d} M^{-\frac{1}{2}} \leqq 1$. We assume further that $4 B_{0} c_{1}\left(d, \frac{1}{4} \gamma_{0}\right)$ $(2 L+1)^{d}|\delta \alpha|_{2}<1$. This assumption is almost the same as $|\delta \alpha|_{2}<\frac{1}{2} \gamma_{1}$, because $\gamma_{1}$ is an inverse of the norm of the operator $G$, and this may be taken as the constant on the right-hand side of the last inequality in (2.11). Now the expression in the first parenthesis can be bounded by $1+\frac{1}{2}+\frac{1}{4}<2$, because $M^{-\frac{1}{2}|\omega|} \leqq 1$ for $|\omega| \geqq 0, M^{-\frac{1}{2}(|\omega|-1)}|\omega| \leqq 1$ for $|\omega| \geqq 1$, and $M^{-\frac{1}{2}(|\omega|-2)} \frac{1}{2}|\omega|$ $(|\omega|-1) \leqq 1$ for $|\omega| \geqq 2$. The expression in the second parenthesis is bounded by $\frac{5}{2} \delta_{1}+\frac{3}{2}|\delta \alpha|_{2}$. Consider the exponential in the third parenthesis and assume that $X_{\omega} \cap X_{1}^{c} \neq \emptyset$, otherwise the exponential is equal to 1 . We combine the exponential with the first exponential on the right-hand side of (2.17), and we would like to estimate the product. At first we estimate it in a general case, for an arbitrary walk $\omega$, and then in the case considered here.

Take a walk $\omega$ and the corresponding sequence of cubes $\left(\square_{z_{0}}, \square_{z_{1}}, \ldots, \square_{z_{n}}\right)$, where $y \in \square_{z_{0}} \cap \mathbb{B}_{k}$. By the definition of $d(y, \omega)$ in (2.11) we take the infimum over a finite set, so it is equal to a value of the function at some elements of the set. Let the sequence $y_{0}=y, y_{1}, \ldots, y_{n}$ be one of them, thus

$$
\begin{aligned}
d(y, \omega) & =d\left(y, y_{1}\right)+d\left(y_{1}, y_{2}\right)+\cdots+d\left(y_{n-1}, y_{n}\right), y_{i} \in \square_{z_{i-1}} \cap \square_{z_{i}} \cap \mathbb{B}_{k}, \\
i & =1, \ldots, n, y_{n} \in \square_{z_{n}} \cap \mathbb{B}_{k} .
\end{aligned}
$$

We have $y_{i}, y_{i+1} \in \square_{z_{i}}$ and we take a path $\Gamma\left(y_{i}, y_{i+1}\right)$ connecting the two points, contained in $\square_{z_{1}}$, and satisfying the equality $d\left(y_{i}, y_{i+1}\right)=\left|\Gamma\left(y_{i}, y_{i+1}\right)\right|_{\mathbb{B}_{k}}$, where the scaled length is defined in (2.4). Combining these paths we get

$$
d(y, \omega)=|\Gamma|_{\mathbb{B}_{k}}, \quad \text { where } \Gamma=\Gamma\left(y, y_{1}\right) \cup \Gamma\left(y_{1}, y_{2}\right) \cup \cdots \cup \Gamma\left(y_{n-1}, y_{n}\right) .
$$

We assume that $\square_{z_{i}} \cap X_{1}^{c} \neq \emptyset$ for some $i$, and we denote by $r$ a minimal $i$ with this property, i.e. $\square_{z_{0}}, \ldots, \square_{z_{r-1}}$ does not intersect $X_{1}^{c}$, and $\square_{z_{r}} \cap X_{1}^{c} \neq \emptyset$. Generally we can have $r=0$. The point $y_{r}$ divides the path $\Gamma$ into two parts, the one from $y$ to $y_{r}$ is denoted by $\Gamma^{0}$, and the other from $y_{r}$ to $y_{n}$ is denoted by $\Gamma^{\prime}$. The index $r$ divides also the walk $\omega$ into two walks: $\omega^{0}=\left(\omega_{0}, \ldots, \omega_{r-1}\right)$, which may be empty, and $\omega^{\prime}=\left(\omega_{r}, \ldots, \omega_{n}\right)$. Obviously we have $X_{\omega} \cap X_{1}^{c}=X_{\omega^{\prime}} \cap X_{1}^{c}$. Let us divide now the path $\Gamma^{\prime}$ into a union of subpaths $\Gamma_{i}, i=1, \ldots, p$, such that

$$
\Gamma^{\prime}=\bigcup_{i=1}^{p} \Gamma_{i}, \quad\left|\Gamma^{\prime}\right|_{\mathbb{B}_{k}}=\sum_{i=1}^{p}\left|\Gamma_{i}\right|_{\mathbb{B}_{k}}, \quad\left|\Gamma_{i}\right|_{\mathbb{B}_{k}}=M \quad \text { for } i<p, \quad\left|\Gamma_{p}\right|_{\mathbb{B}_{k}} \leqq M .
$$

Each path $\Gamma_{i}$ contains some number of points from the sequence $\left(y_{0}, \ldots, y_{n}\right)$; the number may be 0 . Let us fix $\Gamma_{i}$ and denote the points by $y_{s}, y_{s+1}, \ldots, y_{t-1}, s \leqq t$, the set is empty if $s=t$. The points determine the cubes $\square_{z_{s}}, \square_{z_{s+1}}, \ldots, \square_{z_{t-1}}$. The scaled length of the path $\Gamma_{i}$ is $\leqq M$, so it may intersect at most two of the domains $\left\{\Omega_{j} \backslash \Omega_{j+1}\right\}$, and the same is true for the cubes. Therefore there exists an index $j$ such that $\square_{z_{l}} \subset \Omega_{j-1} \backslash \Omega_{j+1}$ for $i=s, s+1, \ldots, t-1$. The ordinary length of $\Gamma_{i}$ in the $\eta$-scale is $\leqq M L^{j} \eta$, hence the projections of $\Gamma_{i}$ onto the coordinate axis are intervals of lengths $\leqq M L^{j} \eta$, and $\Gamma_{i}$ is contained in a continuous space cube $\square_{i}$ of the size $M L^{j} \eta$. Take a cube $\square_{i}^{\prime}$ of the size $7 M L^{j} \eta$, which has the same center as the cube $\square_{i}$. The cubes $\square_{z_{s}}, \ldots, \square_{z_{t-1}}$ have either the size $3 M L^{j} \eta$, or the size $3 M L^{j-1} \eta$, and they have non-empty intersections with $\square_{i}$, hence they are contained in $\square_{i}^{\prime}$. 
Thus the sum in the second exponential in (2.17) for the domain $\square_{z_{s}} \cup \cdots \cup \square \square_{z_{t-1}}$ is reduced to two terms with indices $j-1, j$, and we have

$$
\begin{aligned}
& \frac{1}{\left(M L^{j-1} \eta\right)^{d}}\left|\left(\bigcup_{i=s}^{t-1} \square_{z_{l}}\right) \cap \Omega_{j-1} \cap \Omega_{j}^{c}\right|+\frac{1}{\left(M L^{j} \eta\right)^{d}}\left|\left(\bigcup_{i=s}^{t-1} \square_{z_{l}}\right) \cap \Omega_{j} \cap \Omega_{j+1}^{c}\right| \\
& \leqq \frac{1}{\left(M L^{j-1} \eta\right)^{d}}\left|\square_{i}^{\prime}\right|=\frac{\left(7 M L^{j} \eta\right)^{d}}{\left(M L^{j-1} \eta\right)^{d}}=(7 L)^{d}=\frac{(7 L)^{d}}{M}\left|\Gamma_{i}\right|_{\mathbb{B}_{k}},
\end{aligned}
$$

the last equality holds for $i<p$, for $i=p$ we have simply the bound $(7 L)^{d}$. Summing the above inequalities over $i$ from 1 to $p$ we get

$$
\begin{aligned}
\sum_{j=0}^{k} & \frac{1}{\left(M L^{j} \eta\right)^{d}}\left|X_{\omega^{\prime}} \cap X_{1}^{c} \cap \Omega_{j} \cap \Omega_{j+1}^{c}\right| \\
& \leqq \frac{(7 L)^{d}}{M} \sum_{i=1}^{p-1}\left|\Gamma_{i}\right|_{\mathbb{B}_{k}}+(7 L)^{d} \leqq \frac{(7 L)^{d}}{M}\left|\Gamma^{\prime}\right|_{\mathbb{B}_{k}}+(7 L)^{d} .
\end{aligned}
$$

This is a basic estimate in the general case. We have also $d(y, \omega)=|\Gamma|_{\mathbb{B}_{k}}=$ $\left|\Gamma^{0}\right|_{\mathbb{B}_{k}}+\left|\Gamma^{\prime}\right|_{\mathbb{B}_{k}}$, hence the product of the two exponentials in (2.17) can be bounded as follows:

$$
\begin{aligned}
& e^{-\frac{1}{2} \gamma_{0} d(y, \omega)} \exp \kappa_{1} \sum_{j=1}^{k} \frac{1}{\left(M L^{j} \eta\right)^{d}}\left|X_{\omega} \cap X_{1}^{0} \cap \Omega_{j} \cap \Omega_{j+1}^{c}\right| \\
& \quad \leqq \exp \left(\frac{(7 L)^{d} \kappa_{1}}{M}-\frac{1}{2} \gamma_{0}\right)\left|\Gamma^{\prime}\right|_{\mathbb{B}_{k}} \cdot \exp \left(-\frac{1}{2} \gamma_{0}\left|\Gamma^{0}\right|_{\mathbb{B}_{k}}+(7 L)^{d} \kappa_{1}\right) \\
& \quad \leqq \exp \left(-\frac{1}{2} \gamma_{0}\left|\Gamma^{0}\right|_{\mathbb{B}_{k}}+(7 L)^{d} \kappa_{1}\right), \quad \text { if } \frac{(7 L)^{d} \kappa_{1}}{M}-\frac{1}{2} \gamma_{0} \leqq 0, \\
& \text { or } M \geqq 2(7 L)^{d} \kappa_{1} \frac{1}{\gamma_{0}} .
\end{aligned}
$$

We assume that $M$ satisfies this second restriction, together with the first formulated just after (2.17). We would like to estimate the length $\left|\Gamma^{0}\right|_{\mathbb{B}_{k}}$ by a quantity independent of the walk $\omega$. Let us recall that $\Gamma^{0}$ is a path starting at the point $y$ and ending at the point $y_{r} \in \square_{z_{r-1}} \cap \square_{z_{r}}$, where the cubes $\square_{z_{0}}, \ldots, \square_{z_{r-1}}$ are contained in $X_{1}$, and $\square_{z_{r}}$ intersects both domains $X_{1}, X_{1}^{c}$. From this it follows that all these cubes are from the cover $\pi_{k}^{\prime}$, and that $\square_{z_{r}} \subset\left(X_{1}^{c}\right)^{\sim 2}$, hence $y_{r}$ belongs to this domain and the path $\Gamma^{0}$ connects the point $y$ with the point $y_{r} \in\left(X_{1}^{c}\right)^{\sim 2}$. Thus we have

$$
\left|\Gamma^{0}\right|_{\mathbb{B}_{k}} \geqq d\left(y, y_{r}\right) \geqq \inf _{y^{\prime} \in\left(X_{1}^{c}\right)^{2} \cap \mathbb{B}_{k}} d\left(y, y^{\prime}\right)=d\left(y,\left(X_{1}^{c}\right)^{\sim 2}\right),
$$

and we obtain finally the inequality

$$
\begin{aligned}
& e^{-\frac{1}{2} \gamma_{0} d(y, \omega)} \exp \kappa_{1} \sum_{j=1}^{k} \frac{1}{\left(M L^{j} \eta\right)^{d}}\left|X_{\omega} \cap X_{1}^{c} \cap \Omega_{j} \cap \Omega_{j+1}^{c}\right| \\
& \quad \leqq \exp \left(-\frac{1}{2} \gamma_{0} d\left(y,\left(X_{1}^{c}\right)^{\sim 2}\right)+(7 L)^{d} \kappa_{1}\right), \quad \text { if } X_{\omega} \cap X_{1}^{c} \neq \emptyset .
\end{aligned}
$$


Generally the distance in the last exponential above is equal to 0 , so the exponential can be estimated further only by $\exp (7 L)^{d} \kappa_{1}$.

Consider now the special case of (2.17), when $\Delta(y) \subset \square_{z_{0}}$ and $\square_{z_{0}} \cap X_{0} \neq \emptyset$. Take the cube $\square \in \pi_{k}$ containing $\Delta(y)$, then $\square \subset X_{0}^{\sim 2}, \square^{\sim r} \subset X_{0}^{\sim 2+r}$ and $\square^{\sim r} \cap\left(X_{1}^{c}\right)^{\sim 2}=$ $\emptyset$ if $2+r \leqq r_{0}-2$, or $r \leqq r_{0}-4$, by the condition (2.14). From this we obtain the bound

$$
d\left(y,\left(X_{1}^{c}\right)^{\sim 2}\right)>\left(r_{0}-4\right) M .
$$

In this case the product of the exponentials on the left-hand side of (2.21) can be bounded by

$$
\begin{aligned}
& \exp \left(-\frac{1}{2} \gamma_{0}\left(r_{0}-4\right) M+(7 L)^{d} \kappa_{1}\right) \leqq \exp \left(-\frac{1}{2} \gamma_{0}\left(r_{0}-5\right) M\right) \leqq 1 \\
& \text { if } r_{0} \geqq 5 \text {, which we assume from now on } .
\end{aligned}
$$

Combining all the above estimates, we obtain bounds for the expression in (2.17) for all possible cases, and we obtain the inequalities

$$
\begin{aligned}
\left|\delta \phi_{k}(\delta \alpha, s)\right|< & 2^{d+3} B_{0} c_{1}\left(d, \frac{1}{4} \gamma_{0}\right) e^{(7 L)^{d} \kappa_{1}}\left(\frac{5}{2} \delta_{1}+\frac{3}{2}|\delta \alpha|_{2}\right) \\
\left|\chi_{\Delta(y)} \delta \phi_{k}(\delta \alpha, s)\right|< & 2^{d+3} B_{0} c_{1}\left(d, \frac{1}{4} \gamma_{0}\right)\left(\frac{5}{2} \delta_{1}+\frac{3}{2}|\delta \alpha|_{2}\right) \text { for } \Delta(y) \subset X_{0} \\
\mid \chi_{\Delta(y)}\left(\delta \phi_{k}(\delta \alpha, s)-\right. & \left.\delta \phi_{k}(\delta \alpha, 1)\right) \mid<2^{d+4} B_{0} c_{1}\left(d, \frac{1}{4} \gamma_{0}\right) e^{(7 L)^{d} \kappa_{1}} \\
& \cdot \exp \left(-\frac{1}{2} \gamma_{0} d\left(y,\left(X_{1}^{c}\right)^{2}\right)\right)\left(\frac{5}{2} \delta_{1}+\frac{3}{2}|\delta \alpha|_{2}\right)
\end{aligned}
$$

and the same inequalities for the norms $\left|\partial^{\eta} \cdot\right|_{1},\left|\partial^{\eta} \cdot\right|_{(\alpha), 2-\alpha},\left|\Delta^{\eta} \cdot\right|_{2}$ of the functions on the left-hand sides above. The function $\delta \phi_{k}(\delta \alpha, s)$ has also some localization properties dependent on zeros of $s$, but these will be discussed below.

Consider now the function $\delta \alpha_{k}$ determined by the second equation in (3.20) [3]. It is a non-linear equation, and we construct an analytic extension $\delta \alpha_{k}(s)$ of $\delta \alpha_{k}$ by introducing properly the localization parameters $s$ into this equation. Let us recall that it is

$$
\phi_{0} \cdot \delta \phi_{k}(\delta \alpha)+\frac{1}{2} \delta \phi_{k}(\delta \alpha) \cdot \delta \phi_{k}(\delta \alpha)-\frac{1}{\lambda} \delta \alpha=\frac{1}{\lambda} \alpha_{1}
$$

Unfortunately we cannot do it simply by putting $\delta \phi_{k}(\delta \alpha, s)$ in place of $\delta \phi_{k}(\delta \alpha)$ above, and solving the resulting equation. A problem is a bit subtler, it is a singular equation in $\delta \alpha$, the inverse of the operator determining a linear part of it is unbounded, and we have to use quite a lot of a structure of the function $\delta \phi_{k}(\delta \alpha)$ to deal with this problem. Fortunately enough of this structure is preserved in terms of the random walk expansion (2.13) of this function, so we substitute it in the above equation, and using the definition (2.15) we write it in the form

$$
\begin{gathered}
\sum_{\omega} \phi_{0} \cdot h_{z_{0}} G\left(\square_{z_{0}}, \alpha_{0}\right) h_{z_{0}}(K G)(\tilde{\omega}) g_{\sigma_{0}}+\frac{1}{2} \sum_{\omega^{\prime}, \omega^{\prime \prime}} h_{z_{0}^{\prime}} G\left(\square_{z_{0}^{\prime}}, \alpha_{0}\right) h_{z_{0}^{\prime}}(K G)\left(\tilde{\omega}^{\prime}\right) g_{\sigma_{0}^{\prime}} \\
\cdot h_{z_{0}^{\prime \prime}} G\left(\square_{z_{0}^{\prime \prime}}, \alpha_{0}\right) h_{z_{0}^{\prime \prime}}(K G)\left(\tilde{\omega}^{\prime \prime}\right) g_{\sigma_{0}^{\prime \prime}}-\frac{1}{\lambda} \delta \alpha=\frac{1}{\lambda} \alpha_{1} .
\end{gathered}
$$


The equation has been analyzed in Sect. 3 [3], and we can apply this analysis to the above particular form of the equation without any essential changes, only with some slight changes of constants in the bounds. At first, we apply the operator $G^{-1}\left(\alpha_{0}\right)$ to the above equation and we obtain, after simple transformations and using the identities after (3.33)[3] and the definition (2.6), the following one:

$$
\begin{aligned}
\delta \alpha+ & \frac{1}{\lambda}\left(-\Delta_{\Omega_{1}}^{D, \eta}+Q^{*} a Q+v\right) \delta \alpha+\left(\phi_{0}^{2}-1\right) \delta \alpha+\frac{1}{\lambda} \alpha_{0} \delta \alpha-\phi_{0} \cdot g_{0} \\
& +\sum_{\omega} K\left(h_{z_{0}} \phi_{0}\right) \cdot G\left(\square_{z_{0}}, \alpha_{0}\right) h_{z_{0}}(K G)(\tilde{\omega}) g_{\sigma_{0}}-\sum_{\tilde{\omega}:|\tilde{\omega}|>0} \phi_{0} \cdot(K G)(\tilde{\omega})\left(g_{0}+g_{1}\right) \\
& +\frac{1}{2} \sum_{\omega^{\prime}, \omega^{\prime \prime}} K\left(h_{z_{0}^{\prime \prime}} h_{z_{0}^{\prime}} G\left(\square_{z_{0}^{\prime}}, \alpha_{0}\right)(K G)\left(\tilde{\omega}^{\prime}\right) g_{\sigma_{0}^{\prime}}\right) \cdot G\left(\square_{z_{0}^{\prime \prime}}, \alpha_{0}\right) h_{z_{0}^{\prime \prime}}(K G)\left(\tilde{\omega}^{\prime \prime}\right) g_{\sigma_{0}^{\prime \prime}} \\
& -\frac{1}{2} \sum_{\omega^{\prime}, \tilde{\omega}^{\prime \prime}} h_{z_{0}^{\prime}} G\left(\square_{z_{0}^{\prime}}, \alpha_{0}\right) h_{z_{0}^{\prime}}(K G)\left(\tilde{\omega}^{\prime}\right) g_{\sigma_{0}^{\prime}} \cdot(K G)\left(\tilde{\omega}^{\prime \prime}\right)\left(g_{0}+g_{1}\right) \\
= & -\frac{1}{\lambda}\left(-\Delta_{\Omega_{1}}^{D, \eta}+Q^{*} a Q+v+\alpha_{0}\right) \alpha_{1} .
\end{aligned}
$$

The notation used above for terms of the third sum is rather awkward and does not give justice to simplicity of these expressions. We can rearrange terms of the equation, introduce some new definitions, and write it in a simpler form. Using the condition $|\tilde{\omega}|>0$ we can write $\tilde{\omega}=\left(\omega_{1}, \ldots, \omega_{n}\right), n \geqq 1$, as $\omega^{\prime}=\left(\omega_{0}^{\prime}, \ldots, \omega_{n-1}^{\prime}\right)$, $n-1 \geqq 0$, with obvious identification of elements of the two walks, and we have

$$
\begin{aligned}
& \sum_{\tilde{\omega}:|\tilde{\omega}|>0} \phi_{0} \cdot(K G)(\tilde{\omega})\left(g_{0}+g_{1}\right) \\
& =\sum_{\left(z_{0}^{\prime}, \tilde{\omega}^{\prime}\right)} \phi_{0} K\left(h_{z_{0}^{\prime}}\right) \cdot G\left(\square_{z_{0}^{\prime}}, \alpha_{0}\right) h_{z_{0}^{\prime}}(K G)\left(\tilde{\omega}^{\prime}\right)\left(g_{0}+g_{1}\right) \\
& \quad-\sum_{\left(z_{0}^{\prime}, \tilde{\omega}^{\prime}\right)} \phi_{0} \cdot \delta \alpha h_{z_{0}^{\prime}} G\left(\square_{z_{0}^{\prime}}, \alpha_{0}\right) h_{z_{0}^{\prime}}(K G)\left(\tilde{\omega}^{\prime}\right)\left(g_{0}+g_{1}\right) .
\end{aligned}
$$

We combine the first sum on the right-hand side above with the first sum in (2.26) replacing the operator $K\left(h_{z_{0}} \phi_{0}\right)$ there by $K\left(\phi_{0} h_{z_{0}}\right)-\phi_{0} K\left(h_{z_{0}}\right)$. Let us introduce a new operator $V\left(h_{z}\right)$ acting on a pair of two functions by the following formula:

$$
\begin{aligned}
V\left(h_{z}\right)(f, g)= & K\left(h_{z} f\right) \cdot g-f \cdot K\left(h_{z}\right) g=2 \nabla^{\eta}\left(h_{z} f\right) \cdot \nabla^{\eta} g \\
& -2 f \cdot\left(\nabla^{\eta} h_{z} \cdot \nabla^{\eta} g\right)+2\left(\nabla^{\eta} h_{z} \cdot \nabla^{\eta} f\right) \cdot g+\left(\Delta^{\eta} f\right) \cdot h_{z} g \\
& -\left[Q^{*} a Q, f\right] \cdot h_{z} g=2 \nabla^{\eta} f \cdot \nabla^{\eta}\left(h_{z} g\right)+\left(\Delta^{\eta} f\right) \cdot h_{z} g \\
& -\left[Q^{*} a Q, f\right] \cdot h_{z} g=K(f) \cdot h_{z} g .
\end{aligned}
$$

Notice that the definition is not symmetric in $f, g$, so the operator acts on ordered pairs. Next, we define operators $V_{\omega_{0}}$ for $\omega_{0}=\left(z_{0}, \sigma_{0}\right)$ by the formulas

$$
\begin{array}{ll}
V_{\omega_{0}}(f, g)=V\left(h_{z_{0}}\right)(f, g) & \text { if } \sigma_{0}=0, \\
V_{\omega_{0}}(f, g)=f \cdot \delta \alpha h_{z_{0}} g & \text { if } \sigma_{0}=1,
\end{array}
$$


and we change slightly the definition of the variables $\vartheta_{i}$ in (2.12) by including $\sigma_{0}$ also, i.e.

$$
\vartheta_{i}=0 \quad \text { if } \sigma_{0}+\sigma_{1}+\cdots+\sigma_{i}<2, \quad \vartheta_{i}=1 \quad \text { if } \sigma_{0}+\sigma_{1}+\cdots+\sigma_{i} \geqq 2 .
$$

Thus the first two sums in (2.26) combined can be written as follows:

$$
\sum_{\omega} V_{\omega_{0}}\left(\phi_{0}, G\left(\square_{z_{0}}, \alpha_{0}\right) h_{z_{0}}(K G)(\tilde{\omega})\left(g_{0}+g_{1}\right)\right) .
$$

In the same way we combine the last two sums, except that we have to take the last sum with the restriction $\left|\tilde{\omega}^{\prime \prime}\right|>0$, so we get

$$
\begin{gathered}
\frac{1}{2} \sum_{\omega^{\prime}, \omega^{\prime \prime}: \sigma_{0}^{\prime}=0} V_{\omega_{0}^{\prime \prime}}\left(h_{z_{0}^{\prime}} G\left(\square_{z_{0}^{\prime}}\right) h_{z_{0}^{\prime}}(K G)\left(\tilde{\omega}^{\prime}\right)\left(g_{0}+g_{1}\right), G\left(\square_{z_{0}^{\prime \prime}}, \alpha_{0}\right) h_{z_{0}^{\prime \prime}}(K G)\left(\tilde{\omega}^{\prime \prime}\right)\right. \\
\left.\times\left(g_{0}+g_{1}\right)\right)-\frac{1}{2} \sum_{\omega: \sigma_{0}=0}\left(g_{0}+g_{1}\right) \cdot h_{z_{0}} G\left(\square_{z_{0}}, \alpha_{0}\right) h_{z_{0}}(K G)(\tilde{\omega})\left(g_{0}+g_{1}\right) .
\end{gathered}
$$

Notice that the walks $\omega^{\prime}, \omega^{\prime \prime}$ must begin with points $z_{0}^{\prime}, z_{0}^{\prime \prime}$ such that $\operatorname{supp} h_{z_{0}^{\prime}} \cap$ supp $h_{z_{0}^{\prime \prime}} \neq \emptyset$, which means that either $\square_{z_{0}^{\prime}} \subset \square_{z_{0}^{\prime \prime}}^{\sim}$, or $\square_{z_{0}^{\prime \prime}} \subset \square_{z_{0}^{\prime}}^{\sim}$, or both, depending on the sizes of the cubes. We obtain the equation in which the four sums in (2.26) are replaced by the sum of the above two expressions. The next operation is to apply the operator

$$
\left[1+\frac{1}{\lambda}\left(-\Delta_{\Omega_{1}}^{D, \eta}+Q^{*} a Q+v\right)\right]^{-1}=\lambda G(\lambda)
$$

to both sides of this equation. The operator $G(\lambda)$ is a special case of $G(\alpha)$; we take simply the constant $\alpha=\lambda$, and therefore it has the random walk expansion (2.8). We combine this expansion with the expansions in (2.28), (2.29), and we obtain sums which can be interpreted as sums over random tree graphs of a simple form. We have two classes of tree graphs. The first consists of graphs with a trunk, a vertex, and one branch. The trunk is a random walk $\tau$ of the simplest type appearing in (2.28), the branch is a random walk $\omega$ as in (2.28), and the vertex corresponds to $\omega_{0}$. We denote these tree graphs by $t_{1}$, i.e. $t_{1}=(\tau, \omega)$. The second class is formed by tree graphs with a trunk $\tau$ and two branches $\omega^{\prime}, \omega^{\prime \prime}, \sigma_{0}^{\prime}=0$ and the vertex at $\omega_{0}^{\prime \prime}$. We denote them by $t_{2}$, i.e. $t_{2}=\left(\tau, \omega^{\prime}, \omega^{\prime \prime}\right)$. We have also sums corresponding to simpler graphs, like random walks $\tau$ or $\omega$. Now, to every graph $t$ described above we assign a domain $X_{t}$. This domain is equal to $X_{\tau}$, or $X_{\omega}$, defined in (2.16), if $t=\tau$ or $\omega$. For $t=t_{1}$ we put $X_{t_{1}}=X_{\tau} \cup X_{\omega}$, and for $t=t_{2} X_{t_{2}}=X_{\tau} \cup X_{\omega^{\prime}} \cup X_{\omega^{\prime \prime}}$. We introduce parameters $s$ into the terms of the equation as in the first sum of (2.16), and we obtain the following equation:

$$
\begin{aligned}
\delta \alpha+ & \sum_{\tau} h_{\tau_{0}} \lambda G\left(\square_{\tau_{0}}, \lambda\right) h_{\tau_{0}}(K G(\lambda))(\tilde{\tau})\left(\left(\phi_{0}^{2}-1\right)+\frac{1}{\lambda} \alpha_{0}\right) \delta \alpha \prod_{\square \subset X_{\tau} \cap X_{1}^{c}} s_{\square} \\
& +\sum_{t_{1}=(\tau, \omega)} h_{\tau_{0}} \lambda G\left(\square_{\tau_{0}}, \lambda\right) h_{\tau_{0}}(K G(\lambda))(\tilde{\tau}) V_{\omega_{0}}\left(\phi_{0}, G\left(\square_{z_{0}}, \alpha_{0}\right) h_{z_{0}}(K G)(\tilde{\omega})\right.
\end{aligned}
$$




$$
\begin{aligned}
& \left.\times\left(g_{0}+g_{1}\right)\right) \prod_{\square \subset X_{t_{1}} \cap X_{1}^{c}} s_{\square}+\frac{1}{2} \sum_{t_{2}=\left(\tau, \omega^{\prime}, \omega^{\prime \prime}\right)} h_{\tau_{0}} \lambda G\left(\square_{\tau_{0}}, \lambda\right) h_{\tau_{0}}(K G(\lambda))(\tilde{\tau}) \\
& \times V_{\omega_{0}^{\prime \prime}}\left(h_{z_{0}^{\prime}} G\left(\square_{z_{0}^{\prime}}, \alpha_{0}\right) h_{z_{0}^{\prime}}(K G)\left(\tilde{\omega}^{\prime}\right)\left(g_{0}+g_{1}\right), G\left(\square_{z_{0}^{\prime \prime}}, \alpha_{0}\right) h_{z_{0}^{\prime \prime}}(K G)\left(\tilde{\omega}^{\prime \prime}\right)\left(g_{0}+g_{1}\right)\right) \\
& \times \prod_{\square \subset X_{t_{2}} \cap X_{1}^{c}} s_{\square}-\frac{1}{2} \sum_{t_{1}^{\prime}=\left(\tau, \omega^{\prime}\right)} h_{\tau_{0}} \lambda G\left(\square_{\tau_{0}}, \lambda\right) h_{\tau_{0}} \cdot(K G(\lambda))(\tilde{\tau})\left[\left(g_{0}+g_{1}\right)\right. \\
& \left.=h_{z_{0}^{\prime}} G\left(\square_{z_{0}^{\prime}}, \alpha_{0}\right) h_{z_{0}^{\prime}}(K G)\left(\tilde{\omega}^{\prime}\right)\left(g_{0}+g_{1}\right)\right] \prod_{\square \subset X_{t_{1}^{\prime}} \cap X_{1}^{c}} s_{\square} \\
& =\sum_{\tau} h_{\tau_{0}} \lambda G\left(\square \square_{\tau_{0}}, \lambda\right) h_{\tau_{0}}(K G(\lambda))(\tilde{\tau}) \phi_{0} \cdot g_{0} \prod_{\square X_{\tau} \cap X_{1}^{c}} s_{\square}-\sum_{\tau} h_{\tau_{0}} \lambda G\left(\square \tau_{\tau_{0}}, \lambda\right) \\
& \times h_{\tau_{0}}(K G(\lambda))(\tilde{\tau}) \frac{1}{\lambda}\left(-\Delta_{\Omega_{1}}^{D, \eta}+Q^{*} a Q+v+\alpha_{0}\right) \alpha_{1} \prod_{\square \subset X_{\tau} \cap X_{1}^{c}} s_{\square},
\end{aligned}
$$

where $\omega^{\prime}$ in the third and fourth sums satisfy $\sigma_{0}^{\prime}=0$. Arranging properly terms in the above equation we can write it in the form (3.43) [3], but the functions $H_{0}, H_{1}, H_{2}$ depending on $s$ also. We consider them again on the complex polydisc $\left\{s:|s| \leqq e^{\kappa_{1}}\right\}$. Of course they are analytic functions on this polydisc, and we would like to obtain their bounds. They follow from the basic bounds (2.11), (2.17), and the geometric combinatorial bounds (2.18), (2.19), but we need also additional ones for the operator $\lambda G\left(\square_{z}, \lambda\right)$, ones which are uniform in $\lambda$.

The operator $G\left(\square_{z}, \lambda\right)$ alone satisfies the bound (2.5) with the same constants independent of $\lambda$, and for all $\lambda \geqq 0$, but $\lambda G\left(\square_{z}, \lambda\right)$ does not satisfy such a bound. It is easy to see that this operator approaches 1 if $\lambda \rightarrow+\infty$, so it may satisfy only bounds which hold for the identity operator. We need the following one:

$$
\left(L^{j} \eta\right)^{2}\left|\chi_{\Delta_{J}(y)} \lambda G\left(\square_{z}, \lambda\right) \chi_{\Delta_{J^{\prime}}}\left(y^{\prime}\right) f\right| \leqq B_{0} e^{-\gamma_{0} d\left(y, y^{\prime}\right)}\left(L^{j^{\prime}} \eta\right)^{2}\left|\chi_{\Delta_{J^{\prime}}}\left(y^{\prime}\right) f\right|,
$$

where $f$ is an arbitrary function defined on $\square_{z} \cap \Omega_{1}, y \in \square_{z} \cap \Lambda_{j}, y^{\prime} \in \square_{z} \cap \Lambda_{j^{\prime}}$. This bound may be proved for arbitrary $\lambda \geqq 0$, but for simplicity, we prove it for $\lambda=\lambda_{k}$ only, i.e. for $\lambda$ such that $\lambda \eta^{2} \geqq 1$. The proof follows from several simple remarks. At first notice that $j, j^{\prime}$ may differ at most by $1,\left|j-j^{\prime}\right| \leqq 1$, so (2.31) follows from the same inequality without the scaling factors, with a possible increase of the constant $B_{0}$ by the factor $L^{2}$. Assume that $\square_{z}$ intersects $\Lambda_{j}$, and possibly $\Lambda_{j+1}$, and rescale the operators and the domain to the natural $\xi$-scale, $\xi=L^{-j}$. Then (2.31) follows from the rescaled inequality

$$
\left|\chi_{\Delta_{j}(y)} \lambda^{\prime} G^{\xi}\left(\square_{z}, \lambda^{\prime}\right) \chi_{\Delta_{j^{\prime}}\left(y^{\prime}\right)} f\right| \leqq B_{0}^{\prime} e^{-\gamma_{0} d\left(y, y^{\prime}\right)}\left|\chi_{\Delta_{j^{\prime}}\left(y^{\prime}\right)} f\right|,
$$

where $\lambda^{\prime}=\lambda\left(L^{j} \eta\right)^{2}$. Notice that $d\left(y, y^{\prime}\right)$ is properly rescaled, in particular if $\square_{z}$ intersects $\Lambda_{j}$ only, then $d\left(y, y^{\prime}\right)=\left|y-y^{\prime}\right|_{\xi}$, the distance in the $\xi$-scale. Consider the operator $-\left(\Delta_{\Omega_{1}}^{D, \xi_{1}}\right)_{\square_{z}}^{N}+v^{\prime}+\lambda^{\prime}$, which is equal to the inverse of the operator $G^{\xi}\left(\square_{z}, \lambda^{\prime}\right)$ without $Q^{*} a Q$. Denote its inverse by $C_{0}^{\xi}\left(\square_{z}, \lambda^{\prime}\right)$ and assume that

$$
\left|\chi_{\Delta_{J}(y)} \lambda^{\prime} C_{0}^{\xi}\left(\square_{z}, \lambda^{\prime}\right) \chi_{\Delta_{J^{\prime}}\left(y^{\prime}\right)} f\right| \leqq B_{0}^{\prime \prime} e^{-\gamma_{0}^{\prime}\left|y-y^{\prime}\right|_{\xi}}\left|\chi_{\Delta_{j^{\prime}}\left(y^{\prime}\right)} f\right| .
$$


It is easy to see that the above inequality implies (2.32) for $\lambda^{\prime}$ sufficiently large, because then the following Neumann series is convergent:

$$
\begin{aligned}
\lambda^{\prime} G^{\xi}\left(\square_{z}, \lambda^{\prime}\right)= & \sum_{n=0}^{\infty} \lambda^{\prime} C_{0}^{\xi}\left(\square_{z}, \lambda^{\prime}\right)\left[\left(-\frac{a_{j}}{\lambda^{\prime}} Q_{j}^{*} Q_{j} \chi_{\square_{z} \cap \Omega_{j+1}^{c}}\right.\right. \\
& \left.\left.-\frac{a_{j+1}}{\lambda^{\prime}} L^{-2} Q_{j+1}^{*} Q_{j+1} \chi_{\square_{z} \cap \Omega_{j+1}}\right) \cdot \lambda^{\prime} C_{0}^{\xi}\left(\square_{z}, \lambda^{\prime}\right)\right]^{n} .
\end{aligned}
$$

We can bound it using (2.9), (2.33) and (2.11) with the simpler exponential functions coming from (2.33). Using also the simple inequality $d\left(y, y^{\prime}\right) \leqq\left|y-y^{\prime}\right|_{\xi}$ for $y, y^{\prime} \in \square_{z}$ we obtain (2.32) with $\gamma_{0}=\frac{1}{2} \gamma_{0}^{\prime}, B_{0}^{\prime}=2 B_{0}^{\prime \prime} c_{0}^{d}\left(\frac{1}{2} \gamma_{0}^{\prime}\right)$, if $\frac{3}{2 \lambda^{\prime}} B_{0}^{\prime \prime} c_{0}^{d}\left(\frac{1}{2} \gamma_{0}^{\prime}\right) \leqq \frac{1}{2}$, or $\lambda^{\prime} \geqq 3 B_{0}^{\prime \prime} c_{0}^{d}\left(\frac{1}{2} \gamma_{0}^{\prime}\right)$. If $\lambda^{\prime}<3 B_{0}^{\prime \prime} c_{0}^{d}\left(\frac{1}{2} \gamma_{0}^{\prime}\right)$, then (2.32) follows from the corresponding bound (2.5) for $G^{\xi}\left(\square_{z}, \lambda^{\prime}\right)$ multiplied by the bound for $\lambda^{\prime}$. Thus the inequality (2.31) is reduced to (2.33). This can be rescaled further to the unit lattice, because of our assumption $\lambda^{\prime \prime}=\lambda^{\prime} \xi^{2}=\lambda \eta^{2} \geqq 1$. The rescaled operator is equal to

$$
\begin{aligned}
\lambda^{\prime \prime} C_{0}^{1}\left(\square_{z}, \lambda^{\prime \prime}\right) & =\lambda^{\prime \prime}\left(-\left(\Delta_{\Omega_{1}}^{D, 1}\right)_{\square_{z}}^{N}+v^{\prime \prime}+\lambda^{\prime \prime}\right)^{-1}=\lambda^{\prime \prime}\left(\lambda^{\prime \prime}+v^{\prime \prime}+2 d_{z}-t_{z}\right)^{-1} \\
& =\frac{\lambda^{\prime \prime}}{\lambda^{\prime \prime}+v^{\prime \prime}+2 d_{z}}\left(1-t_{z} \frac{1}{\lambda^{\prime \prime}+v^{\prime \prime}+2 d_{z}}\right)^{-1} \\
& =\frac{\lambda^{\prime \prime}}{\lambda^{\prime \prime}+v^{\prime \prime}+2 d_{z}} \sum_{n=0}^{\infty}\left(t_{z} \frac{1}{\lambda^{\prime \prime}+v^{\prime \prime}+2 d_{z}}\right)^{n}
\end{aligned}
$$

where $2 d_{z}$ and $-t_{z}$ are diagonal and off-diagonal parts of the operator $-\left(\Delta_{\Omega_{1}}^{D, 1}\right)_{\square_{z}}^{N}$. The above series gives the usual random walk expansion of the kernel of the operator, and from this we obtain easily the bound

$$
\begin{aligned}
& \left|\lambda^{\prime \prime} C_{0}^{1}\left(\square_{z}, \lambda^{\prime \prime} ; x, x^{\prime}\right)\right| \\
& \quad \leqq \frac{\lambda^{\prime \prime}}{\lambda^{\prime \prime}+v^{\prime \prime}} \cdot \frac{\lambda^{\prime \prime}+v^{\prime \prime}+2 d}{\lambda^{\prime \prime}+v^{\prime \prime}+d} \exp \left(-\left(\log \frac{\lambda^{\prime \prime}+v^{\prime \prime}+2 d}{2 d}\right)\left|x-x^{\prime}\right|_{1}\right) \\
& \quad<2 e^{-\frac{1}{2 d+1}\left|x-x^{\prime}\right|_{1}} .
\end{aligned}
$$

It implies (2.33) with $\gamma_{0}^{\prime}=\frac{1}{4 d+2}$ and $B_{0}^{\prime \prime}=2 c_{0}^{d}\left(\frac{1}{4 d+2}\right) \exp \left(\frac{L d}{4 d+2}\right)$, which concludes the proof of (2.31).

Let us finish the proof of the inequality (3.41) used in Sect. 3 [3]. We take the random walk expansion (2.8) for $G(\lambda)$, and apply (2.9), (2.11) to it, using the inequalities (2.31) and (2.10) with $\lambda$ instead of $\alpha$. We obtain

$$
|\lambda G(\lambda) f|_{2} \leqq 2^{d+2} B_{0} c_{1}\left(d, \frac{1}{4} \gamma_{0}\right)|f|_{2},
$$

which is the inequality (3.41) [3] with the precisely defined constant.

Now we estimate the expressions in Eq. (2.30) in the norm $|\cdot|_{2}$. We estimate at first the whole sums in (2.30) by polynomials in $|\delta \alpha|_{2}$ and from such inequalities we obtain estimates for the functions $H_{1}(s), H_{2}(s ; \delta \alpha), H_{0}(s)$. We start with the first 
sum in (2.30). Estimating as in (2.11) and using (2.31), (2.19) we obtain $\mid \chi_{\Delta(y)}$ (the first sum) $\left.\right|_{2}$

$$
\begin{aligned}
\leqq & B_{0} c_{1}\left(d, \frac{1}{4} \gamma_{0}\right) \sum_{\tau: y \in \square_{\tau_{0}}} \frac{1}{2^{|\tau|}} \cdot \frac{1}{(2 L+1)^{d|\tau|}} e^{-\frac{1}{2} \gamma_{0} d(y ; \tau)} \cdot M^{-\frac{1}{2}|\tau|} \\
& \times\left(\delta_{0}|\delta \alpha|_{2}+\left|\frac{\alpha_{0}}{\lambda} \delta \alpha\right|_{2}\right) \exp \kappa_{1} \sum_{j=0}^{k} \frac{1}{\left(M L^{j} \eta\right)^{d}}\left|X_{\tau} \cap X_{1}^{c} \cap \Omega_{j} \cap \Omega_{j+1}^{c}\right| \\
\leqq & B_{0} c_{1}\left(d, \frac{1}{4} \gamma_{0}\right) \sum_{\tau: y \in \square_{\tau_{0}}} \frac{1}{2^{|\tau|}} \cdot \frac{1}{(2 L+1)^{d|\tau|}}\left(e^{-\frac{1}{2} \gamma_{0} d\left(y,\left(X_{1}^{c}\right)^{\sim 2}\right)} e^{(7 L)^{d} \kappa_{1}}\right. \\
& \left.\times\left(\text { if } X_{\tau} \cap X_{1}^{c} \neq \emptyset\right)+1\left(\text { if } X_{\tau} \cap X_{1}^{c}=\emptyset\right)\right) M^{-\frac{1}{2}|\tau|}\left(\delta_{0}+\frac{1}{\lambda \eta^{2}}\left|\alpha_{0}\right|_{2}\right)|\delta \alpha|_{2} \\
\leqq & 2^{d+3} B_{0} c_{1}\left(d, \frac{1}{4} \gamma_{0}\right) e^{(7 L)^{d} \kappa_{1}} \delta_{0}|\delta \alpha|_{2} .
\end{aligned}
$$

This holds for all $y \in \mathbb{B}_{k}$. If $\Delta(y) \subset X_{0}$, then we can remove the factor $e^{(7 L)^{d} \kappa_{1}}$ from the bound, which is not important. Again for arbitrary $y$ we have the bound

$\mid\left.\chi_{\Delta(y)}(($ the first sum $)-($ the first sum for $s=1))\right|_{2}$

$$
\leqq 2^{d+4} B_{0} c_{1}\left(d, \frac{1}{4} \gamma_{0}\right) e^{(7 L)^{d} \kappa_{1}} \exp \left(-\frac{1}{2} \gamma_{0} d\left(y,\left(X_{1}^{c}\right)^{\sim 2}\right)\right) \delta_{0}|\delta \alpha|_{2}
$$

which is very important. The two sums on the right-hand side of (2.30) can be estimated in the same way and we obtain the above inequalities for them, but with $2 \delta_{0}|\delta \alpha|_{2}$ replaced by

$$
\begin{gathered}
\left|\phi_{0} \cdot g_{0}\right|_{2}+\left|\frac{1}{\lambda}\left(-\Delta_{\Omega_{1}}^{D, \eta}+Q^{*} a Q+v+\alpha_{0}\right) \alpha_{1}\right|_{2}<\frac{3}{2} \cdot \frac{5}{2} \delta_{1} \\
+\frac{1}{\lambda \eta^{2}}\left(4 d+\frac{1}{6}+1+\delta_{0}\right)\left|\alpha_{1}\right|_{2}<(4 d+5) \delta_{1} .
\end{gathered}
$$

To estimate the second and third sums we have to obtain a bound for the operator $V\left(h_{z}\right)$. From (2.27) we have for $y \in \mathbb{B}_{k}$,

$$
\begin{aligned}
& \left|\chi_{\Delta(y)} V\left(h_{z}\right)(f, g)\right|_{2} \leqq 2 d\left|\chi_{\Delta(y)} \partial f\right|_{1}\left|\chi_{\Delta(y)} \partial\left(h_{z} g\right)\right|_{1} \\
& \quad+\left|\chi_{\Delta(y)} \Delta f\right|_{2}\left|\chi_{\Delta(y)} h_{z} g\right|+d\left|\chi_{\Delta(y)} \partial f\right|_{1}\left|\chi_{\Delta(y)} h_{z} g\right| .
\end{aligned}
$$

For simplicity let us drop the characteristic functions $\chi_{\Delta(y)}$ in the inequalities below, but we always mean the localized inequalities. Taking $f=\phi_{0}$ we get

$$
\begin{aligned}
\left|V\left(h_{z}\right)\left(\phi_{0}, g\right)\right|_{2} \leqq & 2 d\left|\partial \phi_{0}\right|_{1}\left(\left|\partial h_{z} \cdot \eta \partial g\right|_{1}+\left|\left(\partial h_{z}\right) g\right|_{1}+\left|h_{z} \partial g\right|_{1}\right) \\
& +\left|\Delta \phi_{0}\right|_{2}\left|h_{z} g\right|+d\left|\partial \phi_{0}\right|_{1}\left|h_{z} g\right| \\
\leqq & 2 d \delta_{0}\left(\frac{2}{9} M^{-1}|\partial g|_{1}+2 M^{-1}|g|+|\partial g|_{1}\right)+(d+1) \delta_{0}|g| \\
\leqq & (2 d+1) \delta_{0}|\partial g|_{1}+(d+2) \delta_{0}|g| \leqq(2 d+1) \delta_{0}\left(|g|+|\partial g|_{1}\right),
\end{aligned}
$$


where we have used the assumptions (3.21) [3] on $\phi_{0}$. Finally, using the same elementary bounds we get

$$
\left|V\left(h_{z^{\prime \prime}}\right)\left(h_{z^{\prime}} f, g\right)\right|_{2} \leqq(2 d+1)\left(|f|+|\partial f|_{1}+|\Delta f|_{2}\right)\left(|g|+|\partial g|_{1}\right) .
$$

Consider now the second sum in (2.30). Using the estimates (2.11), (2.17), (2.19) we obtain

$\mid\left.\chi_{\Delta(y)}($ the second sum $)\right|_{2}$

$$
\begin{aligned}
\leqq & B_{0} c_{1}\left(d, \frac{1}{4} \gamma_{0}\right) \sum_{t_{1}=(\tau, \omega): y \in \square_{\tau_{0}}} \frac{1}{2^{|\tau|+|\omega|+1}} \cdot \frac{1}{(2 L+1)^{d(|\tau|+|\omega|+1)}} \\
& \cdot\left((2 d+1) \delta_{0}+\frac{3}{2}|\delta \alpha|_{2}\right) 2 B_{0} c_{1}\left(d, \frac{1}{4} \gamma_{0}\right)(2 L+1)^{d} e^{-\frac{1}{2} \gamma_{0} d\left(y ; t_{1}\right)} \\
& \times M^{-\frac{1}{2}|\tau|}\left[M^{-\frac{1}{2}|\omega|}+|\omega| M^{-\frac{1}{2}(|\omega|-1)} 2 B_{0} c_{1}\left(d, \frac{1}{4} \gamma_{0}\right)(2 L+1)^{d}|\delta \alpha|_{2} \theta_{+}(|\omega|-1)\right. \\
& \left.+\frac{1}{2}|\omega|(|\omega|-1) M^{-\frac{1}{2}(|\omega|-2)}\left(2 B_{0} c_{1}\left(d, \frac{1}{4} \gamma_{0}\right)(2 L+1)^{d}|\delta \alpha|_{2}\right)^{2} \theta_{+}(|\omega|-2)\right] \\
& \cdot\left(\frac{5}{2} \delta_{1}+\frac{3}{2}|\delta \alpha|_{2}\right) \exp \kappa_{1} \sum_{j=0}^{k} \frac{1}{\left(M L^{j} \eta\right)^{d}}\left|X_{t_{1}} \cap X_{1}^{c} \cap \Omega_{j} \cap \Omega_{j+1}^{c}\right| \\
\leqq & B_{0} c_{1}\left(d, \frac{1}{4} \gamma_{0}\right) \cdot 2 B_{0} c_{1}\left(d, \frac{1}{4} \gamma_{0}\right)(2 L+1)^{d} e^{(7 L)^{d} \kappa_{1}} \cdot\left((2 d+1) \delta_{0}+\frac{3}{2}|\delta \alpha|_{2}\right) \\
& \cdot\left(\frac{5}{2} \delta_{1}+\frac{3}{2}|\delta \alpha|_{2}\right)\left[1+2 B_{0} c_{1}\left(d, \frac{1}{4} \gamma_{0}\right)(2 L+1)^{d}|\delta \alpha|_{2}\right. \\
& \left.+\left(2 B_{0} c_{1}\left(d, \frac{1}{4} \gamma_{0}\right)(2 L+1)^{d}|\delta \alpha|_{2}\right)^{2}\right] \cdot \sum_{t_{1}=(\tau, \omega): y \in \square_{\tau_{0}}} \frac{1}{2^{\left|t_{1}\right|}} \\
& \cdot \frac{1}{(2 L+1)^{d\left|t_{1}\right|}\left(\exp \left(-\frac{1}{2} \gamma_{0} d\left(y,\left(X_{1}^{c}\right)^{\sim 2}\right)+(7 L)^{d} \kappa_{1}\right)\left(\text { if } X_{t_{1}} \cap X_{1}^{c} \neq \emptyset\right) .\right.} \\
& \left.+1\left(\text { if } X_{t_{1}} \cap X_{1}^{c}=\emptyset\right)\right) \leqq 2^{d+2} B_{0} c_{1}\left(d, \frac{1}{4} \gamma_{0}\right) B_{3}\left((2 d+1) \delta_{0}+\frac{3}{2}|\delta \alpha|_{2}\right) \\
& \times\left(\frac{5}{2} \delta_{1}+\frac{3}{2}|\delta \alpha|_{2}\right)\left(1+B_{2}|\delta \alpha|_{2}+B_{2}^{2}|\delta \alpha|_{2}^{2}\right),
\end{aligned}
$$

where we have denoted $\left|t_{1}\right|=|\tau|+|\omega|+1$, and

$$
\begin{aligned}
& B_{2}=2 B_{0} c_{1}\left(d, \frac{1}{4} \gamma_{0}\right)(2 L+1)^{d}, \\
& B_{3}=2 B_{0} c_{1}\left(d, \frac{1}{4} \gamma_{0}\right)(2 L+1)^{d} e^{(7 L)^{d} \kappa_{1}}=B_{2} e^{(7 L)^{d} \kappa_{1}} .
\end{aligned}
$$

The last bound holds for all $y \in \mathbb{B}_{k}$. The difference of the second sum and this sum with $s=1$ can be estimated by the right-hand side above multiplied by $2 \exp \left(-\frac{1}{2} \gamma_{0} d\left(y,\left(X_{1}^{c}\right)^{\sim 2}\right)\right)$. 
The fourth sum in (2.30) has the same form as the second sum, but the operator $V_{\omega_{0}}$ in the vertex is replaced by a simpler multiplication operator by the function $g_{0}+g_{1}$. Therefore this sum can be bounded as in (2.39), but with the factor $(2 d+1) \delta_{0}+\frac{3}{2}|\delta \alpha|_{2}$ replaced by $\frac{5}{2} \delta_{1}+\frac{3}{2}|\delta \alpha|_{2}$, i.e. we have

$$
\begin{aligned}
& \mid\left.\chi_{\Delta(y)}(\text { the fourth sum })\right|_{2} \leqq 2^{d+1} B_{0} c_{1}\left(d, \frac{1}{4} \gamma_{0}\right) B_{3}\left(\frac{5}{2} \delta_{1}+\frac{3}{2}|\delta \alpha|_{2}\right)^{2} \\
& \left(1+B_{2}|\delta \alpha|_{2}+B_{2}^{2}|\delta \alpha|_{2}^{2}\right) .
\end{aligned}
$$

The difference of the sum and the sum with $s=1$ can be estimated again by the above bound multiplied by $2 \exp \left(-\frac{1}{2} \gamma_{0} d\left(y,\left(X_{1}^{c}\right)^{\sim 2}\right)\right)$.

Consider finally the third sum in (2.30). It has a more complicated structure, the summation is not just over random walks, but over tree graphs with one vertex and two branches. We estimate it again as in (2.11), (2.17), (2.39), and we obtain the following preliminary bound:

$$
\begin{aligned}
& \mid\left.\chi_{\Delta(y)}(\text { the third sum })\right|_{2} \\
& \leqq \frac{1}{2} B_{0} c_{1}\left(d, \frac{1}{4} \gamma_{0}\right)\left(2 d+1+|\delta \alpha|_{2}\right) 2\left(2 B_{0} c_{1}\left(d, \frac{1}{4} \gamma_{0}\right)(2 L+1)^{d}\right)^{2} \\
& \cdot\left(1+B_{2}|\delta \alpha|_{2}+B_{2}^{2}|\delta \alpha|_{2}^{2}\right)^{2}\left(\frac{5}{2} \delta_{1}+\frac{3}{2}|\delta \alpha|_{2}\right)^{2} \sum_{t_{2}=\left(\tau, \omega^{\prime}, \omega^{\prime \prime}\right): y \in \square_{\tau_{0}}} \frac{1}{2^{\left|t_{2}\right|}} \\
& \cdot \frac{1}{(2 L+1)^{d\left|t_{2}\right|}} \cdot e^{-\frac{1}{2} \gamma_{0} d\left(y, t_{2}\right)} \exp \kappa_{1} \sum_{j=0}^{k} \frac{1}{\left(M L^{j} \eta\right)^{d}}\left|X_{t_{2}} \cap X_{1}^{c} \cap \Omega_{j} \cap \Omega_{j+1}^{c}\right| \text {, }
\end{aligned}
$$

where $\left|t_{2}\right|=|\tau|+\left|\omega^{\prime}\right|+\left|\omega^{\prime \prime}\right|+2$, and $d\left(y ; t_{2}\right)$ is defined in the same way as $d(y ; \omega)$ in (2.11), but now we take points along two branches $\omega^{\prime}, \omega^{\prime \prime}$ of $t_{2}$. It is easy to see that there is a tree graph $\Gamma$ in the domain $X_{t_{2}}$, consisting of a path starting at $y$ and ending at some point $y_{n}$ of the last cube in $\tau$, and of two paths starting at $y_{n}$ and ending at points of the last cubes in $\omega^{\prime}, \omega^{\prime \prime}$, such that $d\left(y ; t_{2}\right)=|\Gamma|_{\mathbb{B}_{k}}$. We decompose the graph $\Gamma$ into two disjoint parts $\Gamma^{0}, \Gamma^{\prime}$ as in the proof of (2.19), and applying this inequality to the two branches of $\Gamma^{\prime}$ we obtain an inequality of the form (2.19), but with the term $(7 L)^{d} \kappa_{1}$ in the last exponential multiplied by 2 . Thus in a general case we obtain the bound

$$
\begin{aligned}
\mid\left.\chi_{\Delta(y)}(\text { the third term })\right|_{2} \leqq & 2^{d+2} B_{0} c_{1}\left(d, \frac{1}{4} \gamma_{0}\right) B_{3}^{2}\left(2 d+1+|\delta \alpha|_{2}\right) \\
& \times\left(\frac{5}{2} \delta_{1}+\frac{3}{2}|\delta \alpha|_{2}\right)^{2} \cdot\left(1+B_{2}|\delta \alpha|_{2}+B_{2}^{2}|\delta \alpha|_{2}^{2}\right)^{2} .
\end{aligned}
$$

If $X_{t_{2}}$ intersects $X_{1}^{c}$, then we can choose a maximal path in $\Gamma^{0}$, and the arguments leading to the inequalities (2.20), (2.21) can be applied to this maximal path. 
Thus the difference of the third sum and the third sum for $s=1$ can be estimated by the right-hand side of (2.42) multiplied again by $2 \exp \left(-\frac{1}{2} \gamma_{0} d\left(y,\left(X_{1}^{c}\right)^{\sim 2}\right)\right)$.

Equation (2.30) can be written in the form

$$
\delta \alpha+H_{1}(s) \delta \alpha+H_{2}(s ; \delta \alpha)=H_{0}(s),
$$

where the functions $H_{0}, H_{1}, H_{2}$ are defined by gathering together terms in (2.30) of the corresponding orders in $\delta \alpha$. We obtain bounds for these functions by gathering together terms of the corresponding orders in $|\delta \alpha|_{2}$ in bounds discussed above. For simplicity we introduce the following preliminary restrictions on $\delta_{0}, \delta_{1},|\delta \alpha|_{2}$ :

$$
B_{2} \delta_{0} \leqq \frac{1}{10}, \quad B_{2} B_{3} \delta_{1} \leqq \frac{1}{10}, \quad B_{2}|\delta \alpha|_{2} \leqq \frac{1}{10} .
$$

Our final restrictions will be much stronger. Using these restrictions we obtain, after some simple estimates, the inequalities

$$
\begin{aligned}
\left|H_{0}(s)\right|_{2} & <B_{3} \delta_{1},\left|\chi_{\Delta(y)}\left(H_{0}(s)-H_{0}(1)\right)\right|_{2}<\exp \left(-\frac{1}{2} \gamma_{0} d\left(y,\left(X_{1}^{c}\right)^{\sim 2}\right)\right) B_{3} \delta_{1} \\
\left|H_{1}(s) \delta \alpha\right|_{2} & \leqq B_{1}\left(\delta_{0}+\delta_{1}\right)|\delta \alpha|_{2},\left|\chi_{\Delta(y)}\left(H_{1}(s) \delta \alpha-H_{1}(1) \delta \alpha\right)\right|_{2} \\
& \leqq \exp \left(-\frac{1}{2} \gamma_{0} d\left(y,\left(X_{1}^{c}\right)^{\sim 2}\right)\right) B_{1}\left(\delta_{0}+\delta_{1}\right)|\delta \alpha|_{2} \\
\left|H_{2}(s ; \delta \alpha)\right|_{2} & \leqq B_{1}|\delta \alpha|_{2}^{2},\left|\chi_{\Delta(y)}\left(H_{2}(s ; \delta \alpha)-H_{2}(1 ; \delta \alpha)\right)\right|_{2} \\
& \leqq \exp \left(-\frac{1}{2} \gamma_{0} d\left(y,\left(X_{1}^{c}\right)^{2}\right)\right) B_{1}|\delta \alpha|_{2}^{2}
\end{aligned}
$$

where we may take $B_{1}=27 B_{2} B_{3}^{2}$. The functions above are analytic functions of $s$ and $\delta \alpha$ on the spaces $\left\{s:|s| \leqq e^{\kappa_{1}}\right\}$ and $\left\{\delta \alpha:|\delta \alpha|_{2} \leqq \gamma_{2}\right\}$, where $\gamma_{2}=$ $\min \left\{\frac{1}{10} B_{2}^{-1}, \frac{1}{2} \gamma_{1}\right\}$. We repeat the reasoning leading to Lemma 3.2 [3], and we conclude that Eq. (2.43) has a unique solution in $\left\{\delta \alpha:|\delta \alpha|_{2}<c\right\}$ if $B_{3} \delta_{1} \leqq \frac{1}{2} c, B_{1} \delta_{0} \leqq$ $\frac{1}{8}, B_{1} c \leqq \frac{1}{8}, c \leqq \gamma_{2}$. A maximal such $c$ is denoted by $c_{6}$, and is given by $c_{6}=$ $\min \left\{\frac{1}{8} B_{1}^{-1}, \gamma_{2}\right\}$. The solution $\delta \alpha_{k}(s)$ is an analytic function of $s, \phi_{0}, \alpha_{0}, \delta \psi, f_{1}, \alpha_{1}$ on the corresponding domains, and it satisfies the bound

$$
\left|\delta \alpha_{k}(s)\right|_{2} \leqq 2\left|H_{0}(s)\right|_{2}<2 B_{3} \delta_{1} .
$$

This ends the construction of the "localization extension" of the function $\delta \alpha_{k}$.

We also need a bound for the difference $\delta \alpha(s)-\delta \alpha(1)$ of a type of the second bound in (2.44). We subtract Eqs. (2.43) for $\delta \alpha(s), \delta \alpha(1)$, and we obtain the following equation for the difference:

$$
\begin{aligned}
\left(\delta \alpha_{k}(s)-\delta \alpha_{k}(1)\right)+H_{1}(1)\left(\delta \alpha_{k}(s)-\delta \alpha_{k}(1)\right) \\
\quad+\int_{0}^{1} d t\left\langle\left(\frac{\delta}{\delta(\delta \alpha)} H_{2}\right)\left(1 ; t \delta \alpha_{k}(s)+(1-t) \delta \alpha_{k}(1)\right),\left(\delta \alpha_{k}(s)-\delta \alpha_{k}(1)\right)\right\rangle \\
=\left(H_{0}(s)-H_{0}(1)\right)-\left(H_{1}(s) \delta \alpha_{k}(s)-H_{1}(1) \delta \alpha_{k}(s)\right) \\
\quad-\left(H_{2}\left(s ; \delta \alpha_{k}(s)\right)-H_{2}\left(1 ; \delta \alpha_{k}(s)\right)\right) .
\end{aligned}
$$

In order to be able to use the inequalities for the differences in (2.44) we have to localize the above equation, i.e. we multiply it by $\chi_{\Delta(y)}, y \in \mathbb{B}_{k}$, and we introduce the decomposition $1=\sum_{y^{\prime} \in \mathbb{B}_{k}} \chi_{\Delta\left(y^{\prime}\right)}$ before the differences $\delta \alpha(s)-\delta \alpha(1)$ in the 
second and third terms on the left-hand side. We have to obtain bounds for the localized expressions $\chi_{\Delta(y)} H_{1}(s) \chi_{\Delta\left(y^{\prime}\right)} \delta \tilde{\alpha}$ and $\chi_{\Delta(y)}\left\langle\left(\frac{\delta}{\delta(\delta \alpha)} H_{2}\right)(s ; \delta \alpha), \chi_{\Delta\left(y^{\prime}\right)} \delta \tilde{\alpha}\right\rangle$. We need them for $s=1$ only, but we use all the bounds proved until now for the general admissible $s$, so we may consider these general expressions also. Let us start with the operator $H_{1}(s)$. The function $H_{1}(s) \chi_{\Delta\left(y^{\prime}\right)} \delta \tilde{\alpha}$ is represented as a sum of terms over the class of random tree graphs $t$ described before. We estimate it making only a few simple changes in the proof of the third inequality in (2.44). The basic steps yielding the exponential factors for each term are described in (2.9), (2.11). We replace each factor by a product of three factors, one with $\gamma_{0}$ replaced by $\frac{1}{2} \gamma_{0}$, and two with $\gamma_{0}$ replaced by $\frac{1}{4} \gamma_{0}$. The product of the factors with $\frac{1}{2} \gamma_{0}$ is bounded by $\exp \left(-\frac{1}{2} \gamma_{0} d\left(y, y^{\prime}\right)\right)$, one product of the factors with $\frac{1}{4} \gamma_{0}$ is bounded by $\exp \left(-\frac{1}{4} \gamma_{0} d(y, t)\right)$, where $t$ is the graph connected with the term, and the remaining factors with $\frac{1}{4} \gamma_{0}$ are used to control the sum over the sequences of points $\left(y_{i}\right)$, as in (2.11). We obtain the same bounds as before, but with the constants $c_{1}\left(d, \frac{1}{8} \gamma_{0}\right)$ instead of $c_{1}\left(d, \frac{1}{4} \gamma_{0}\right)$, and with the condition on $M$ stronger than the condition in (2.19) by the factor 2. Also, there is no summation over $y^{\prime}$, hence one factor $c_{1}\left(d, \frac{1}{8} \gamma_{0}\right)$ is missing. Thus we obtain the inequality

$$
\begin{aligned}
& \left|\chi_{\Delta(y)} H_{1}(s) \chi_{\Delta\left(y^{\prime}\right)} \delta \tilde{\alpha}\right|_{2} \\
& \quad \leqq \exp \left(-\frac{1}{2} \gamma_{0} d\left(y, y^{\prime}\right)\right) B_{1} c_{1}^{-1}\left(d, \frac{1}{8} \gamma_{0}\right)\left(\delta_{0}+\delta_{1}\right)\left|\chi_{\Delta\left(y^{\prime}\right)} \delta \tilde{\alpha}\right|_{2} .
\end{aligned}
$$

For $s=1$ the constant $B_{1}$ may be improved by replacing the constant $B_{3}$ by $B_{2}$ in all the estimates, but it is not important. Consider now the second expression, the differential of $\mathrm{H}_{2}$. The function $\mathrm{H}_{2}$ is also a sum of terms of the class of random tree graphs, but now we have at least two vertices with the function $\delta \alpha$, and at most seven, including the expressions at end-points of the graphs. The differentiation replaces one of the functions $\delta \alpha$ by $\chi_{\Delta\left(y^{\prime}\right)} \delta \tilde{\alpha}$, or one of the operators $G\left(\square_{z}, \alpha_{0}+\right.$ $\delta \alpha$ ) by its differential equal to $-G\left(\square_{z}, \alpha_{0}+\delta \alpha\right) \chi_{\Delta\left(y^{\prime}\right)} \delta \tilde{\alpha} G\left(\square_{z}, \alpha_{0}+\delta \alpha\right)$. The first operation results in an increase of the number of terms by the factor 7 at most, each term is bounded in the same way as in the proof of (2.47). The second operation results in replacing the terms $\frac{1}{2}|\omega|(|\omega|-1) \cdot M^{-\frac{1}{2}(|\omega|-2)}\left(B_{2}|\delta \alpha|_{2}\right)^{2}$ in the bounds (2.39), (2.40), (2.41) by

$$
\frac{1}{6}(|\omega|+1)|\omega|(|\omega|-1) M^{-\frac{1}{2}(|\omega|-2)}\left(B_{2}|\delta \alpha|_{2}\right)^{2} B_{0}\left|\chi_{\Delta\left(y^{\prime}\right)} \delta \tilde{\alpha}\right|_{2} \leqq B_{2}^{2}|\delta \alpha|_{2}^{2} B_{0}\left|\chi_{\Delta\left(y^{\prime}\right)} \delta \tilde{\alpha}\right|_{2},
$$

and the rest of the bound is again the same as in the proof of (2.47). We obtain a bound in which $B_{1}|\delta \alpha|_{2}$ is replaced by $\left(70 B_{1} B_{2} c_{1}^{-1}\left(d, \frac{1}{8} \gamma_{0}\right)+B_{1} B_{0}\right)\left|\chi_{\Delta\left(y^{\prime}\right)} \delta \tilde{\alpha}\right|_{2}$, and we denote the new constant by $B_{1} c_{1}^{-1}\left(d, \frac{1}{8} \gamma_{0}\right)$. Obviously this new $B_{1}$ is bigger than the one in (2.44). We obtain the inequality

$$
\begin{aligned}
& \left|\chi_{\Delta(y)}\left\langle\left(\frac{\delta}{\delta(\delta \alpha)} H_{2}\right)(s ; \delta \alpha), \chi_{\Delta\left(y^{\prime}\right)} \delta \tilde{\alpha}\right\rangle\right|_{2} \\
& \quad \leqq \exp \left(-\frac{1}{2} \gamma_{0} d\left(y, y^{\prime}\right)\right) B_{1} c_{1}^{-1}\left(d, \frac{1}{8} \gamma_{0}\right)|\delta \alpha|_{2}\left|\chi_{\Delta\left(y^{\prime}\right)} \delta \tilde{\alpha}\right|_{2} .
\end{aligned}
$$

Notice that the third and fifth inequalities in (2.44), follow from (2.47), (2.48).

Now we are ready to estimate the difference $\delta \alpha_{k}(s)-\delta \alpha_{k}(1)$ using the localized equation (2.46). We take the norm $|\cdot|_{2}$ of both sides, and multiply by 
$\exp \frac{1}{4} \gamma_{0} d\left(y,\left(X_{1}^{c}\right)^{\sim 2}\right)$. The norm of the left-hand side is bounded from below by the norm of the first term minus the norms of the second and third terms. We estimate the norm of the second term as follows:

$$
\begin{aligned}
& \exp \frac{1}{4} \gamma_{0} d\left(y,\left(X_{1}^{c}\right)^{\sim 2}\right)\left|\sum_{y^{\prime} \in \mathbb{B}_{k}} \chi_{\Delta(y)} H_{1}(1) \chi_{\Delta\left(y^{\prime}\right)}\left(\delta \alpha_{k}(s)-\delta \alpha_{k}(1)\right)\right|_{2} \\
& \leqq \sum_{y^{\prime} \in \mathbb{B}_{k}} \exp \frac{1}{4} \gamma_{0} d\left(y,\left(X_{1}^{c}\right)^{\sim 2}\right) \exp \left(-\frac{1}{2} \gamma_{0} d\left(y, y^{\prime}\right)\right) \\
& \times \exp \left(-\frac{1}{4} \gamma_{0} d\left(y^{\prime},\left(X_{1}^{c}\right)^{\sim 2}\right)\right) B_{1} c_{1}^{-1}\left(d, \frac{1}{8} \gamma_{0}\right)\left(\delta_{0}+\delta_{1}\right) \\
& \text { - } \exp \frac{1}{4} \gamma_{0} d\left(y^{\prime},\left(X_{1}^{c}\right)^{\sim 2}\right)\left|\chi_{\Delta\left(y^{\prime}\right)}\left(\delta \alpha_{k}(s)-\delta \alpha_{k}(1)\right)\right|_{2} \\
& \leqq \sum_{y^{\prime} \in \mathbb{B}_{k}} \exp \left(-\frac{1}{4} \gamma_{0} d\left(y, y^{\prime}\right)\right) B_{1} c_{1}^{-1}\left(d, \frac{1}{8} \gamma_{0}\right)\left(\delta_{0}+\delta_{1}\right) \\
& \times \exp \frac{1}{\dot{4}} \gamma_{0} d\left(y^{\prime},\left(X_{1}^{c}\right)^{\sim 2}\right)\left|\chi_{\Delta\left(y^{\prime}\right)}\left(\delta \alpha_{k}(s)-\delta \alpha_{k}(1)\right)\right|_{2} \\
& \leqq \frac{3}{16} \sup _{y} \exp \frac{1}{4} \gamma_{0} d\left(y,\left(X_{1}^{c}\right)^{\sim 2}\right)\left|\chi_{\Delta(y)}\left(\delta \alpha_{k}(s)-\delta \alpha_{k}(1)\right)\right|_{2},
\end{aligned}
$$

where we have used the conditions $B_{1} \delta_{0} \leqq \frac{1}{8}, B_{1} B_{3} \delta_{1} \leqq \frac{1}{2} B_{1} c_{6} \leqq \frac{1}{16}$, which imply $B_{1}\left(\delta_{0}+\delta_{1}\right)<\frac{1}{8}+\frac{1}{16}=\frac{3}{16}$. We estimate in the same way the norm of the third term using the inequality (2.48), and we bound it by

$$
\begin{aligned}
& B_{1} \sup _{t \in[0,1]}\left|t \delta \alpha_{k}(s)+(1-t) \delta \alpha_{k}(1)\right|_{2} \\
& \quad \cdot \sup _{y} \exp \frac{1}{4} \gamma_{0} d\left(y,\left(X_{1}^{c}\right)^{\sim 2}\right)\left|\chi_{\Delta(y)}\left(\delta \alpha_{k}(s)-\delta \alpha_{k}(1)\right)\right|_{2} .
\end{aligned}
$$

The first supremum is bounded by $2 B_{3} \delta_{1}$, and $2 B_{1} B_{3} \delta_{1} \leqq \frac{1}{8}$, hence the above expression can be bounded by $\frac{1}{8}$ times the second supremum. Thus the supremum over $y \in \mathbb{B}_{k}$ of the norm of the left-hand side is bounded from below by $1-\frac{3}{16}-\frac{1}{8}=\frac{11}{16}$ times the second supremum above. Using the second, fourth and sixth inequalities in (2.44) we bound the norm of the right-hand side by

$$
\begin{aligned}
& B_{3} \delta_{1}+B_{1}\left(\delta_{0}+\delta_{1}\right)\left|\delta \alpha_{k}(s)\right|_{2}+B_{1}\left|\delta \alpha_{k}(s)\right|_{2}^{2} \\
& \quad<B_{3} \delta_{1}+B_{1}\left(\delta_{0}+\delta_{1}\right) 2 B_{3} \delta_{1}+4 B_{1} B_{3}^{2} \delta_{1}^{2}<\frac{13}{8} B_{3} \delta_{1} .
\end{aligned}
$$

Combining the bounds we obtain

$$
\left|\chi_{\Delta(y)}\left(\delta \alpha_{k}(s)-\delta \alpha_{k}(1)\right)\right|_{2}<3 \exp \left(-\frac{1}{4} \gamma_{0} d\left(y,\left(X_{1}^{c}\right)^{\sim 2}\right)\right) B_{3} \delta_{1} .
$$


The two bounds (2.45), (2.49) are the basic bounds for the solution $\delta \alpha(s)$ of the equation (2.43). It also has the following fundamental localization property: if $s=0$ on a family $\mathscr{C}$ of cubes $\square$, then $\delta \alpha_{k}(s)=0$ on the domain $\bigcup_{\square \in \mathscr{C}} \square$, and $\delta \alpha_{k}(s)$ does not depend on $\phi_{0}, \alpha_{0}, \delta \psi, f_{1}, \alpha_{1}$ restricted to this domain. This property follows immediately from the form (2.30) of the equation.

Let us come back to the function $\delta \phi_{k}(\delta \alpha, s)$. For $s=1$, it becomes a solution of Eqs. (3.20) [3] if we substitute $\delta \alpha_{k}$ (1) instead of $\delta \alpha$. Generally we substitute the solution $\delta \alpha_{k}(s)$ instead of $\delta \alpha$, and we denote $\delta \phi_{k}(s)=\delta \phi_{k}\left(\delta \alpha_{k}(s), s\right)$. From the inequalities (2.23) we obtain

$$
\begin{gathered}
\left|\delta \phi_{k}(s)\right|<2^{d+3} B_{0} c_{1}\left(d, \frac{1}{4} \gamma_{0}\right) e^{(7 L)^{d} \kappa_{1}}\left(\frac{5}{2}+3 B_{3}\right) \delta_{1}<B_{3}^{2} \delta_{1}, \\
\left|\chi_{\Delta(y)}\left(\delta \phi_{k}(s)-\delta \phi_{k}\left(\delta \alpha_{k}(s), 1\right)\right)\right|<\exp \left(-\frac{1}{2} \gamma_{0} d\left(y,\left(X_{1}^{c}\right)^{\sim 2}\right)\right) B_{3}^{2} \delta_{1},
\end{gathered}
$$

and the same inequalities for the other norms listed after (2.23). To estimate the difference $\delta \phi_{k}(s)-\delta \phi_{k}(1)$ we have to estimate the difference

$$
\begin{aligned}
& \delta \phi_{k}\left(\delta \alpha_{k}(s), 1\right)-\delta \phi_{k}\left(\delta \alpha_{k}(1), 1\right) \\
& \quad=\int_{0}^{1} d t\left\langle\left(\frac{\delta}{\delta(\delta \alpha)} \delta \phi_{k}\right)\left(t \delta \alpha_{k}(s)+(1-t) \delta \alpha_{k}(1), 1\right), \delta \alpha_{k}(s)-\delta \alpha_{k}(1)\right\rangle .
\end{aligned}
$$

We do it in the same way as for (2.46), we estimate the localized differential $\chi_{\Delta(y)}\left\langle\left(\frac{\delta}{\delta(\delta \alpha)} \delta \phi_{k}\right)(\delta \alpha, s), \chi_{\Delta\left(y^{\prime}\right)} \delta \tilde{\alpha}\right\rangle$. The function $\delta \phi_{k}(\delta \alpha, s)$ is given by the random walk expansion (2.16) with at most three functions $\delta \alpha$ at vertices of the walks. The differential is obtained by replacing one of the functions $\delta \alpha$ by $\chi_{\Delta\left(y^{\prime}\right)} \delta \tilde{\alpha}$, or one of the operators $G\left(\square_{z}, \alpha_{0}+\delta \alpha\right)$ by $-G\left(\square_{z}, \alpha_{0}+\delta \alpha\right) \chi_{\Delta\left(y^{\prime}\right)} \delta \tilde{\alpha} G\left(\square_{z}, \alpha_{0}+\delta \alpha\right)$. We estimate it in exactly the same way as in the proof of (2.48). In this simpler case we can do it a bit more carefully, as in the inequality (2.17), and we obtain the following bound:

$$
\begin{aligned}
& \left|\chi_{\Delta(y)}\left\langle\left(\frac{\delta}{\delta(\delta \alpha)} \delta \phi_{k}\right)(\delta \alpha, s), \chi_{\Delta\left(y^{\prime}\right)} \delta \tilde{\alpha}\right\rangle\right| \\
& \quad \leqq \exp \left(-\frac{1}{2} \gamma_{0} d\left(y, y^{\prime}\right)\right) B_{3} c_{1}^{-1}\left(d, \frac{1}{8} \gamma_{0}\right)\left|\chi_{\Delta\left(y^{\prime}\right)} \delta \tilde{\alpha}\right|_{2}
\end{aligned}
$$

holding for all $\delta \alpha$ in the space $\left\{\delta \alpha:|\delta \alpha|_{2}<\gamma_{2}\right\}$. Combining (2.51), (2.52) and (2.49) we obtain

$$
\begin{aligned}
& \left|\chi_{\Delta(y)}\left(\delta \phi_{k}\left(\delta \alpha_{k}(s), 1\right)-\delta \phi_{k}\left(\delta \alpha_{k}(1), 1\right)\right)\right| \\
& \quad \leqq \sum_{y^{\prime}} \exp \left(-\frac{1}{2} \gamma_{0} d\left(y, y^{\prime}\right)\right) B_{3} c_{1}^{-1}\left(d, \frac{1}{8} \gamma_{0}\right) \cdot \exp \left(-\frac{1}{4} \gamma_{0} d\left(y^{\prime},\left(X_{1}^{c}\right)^{\sim 2}\right)\right) 3 B_{3} \delta_{1} \\
& \quad \leqq \exp \left(-\frac{1}{4} \gamma_{0} d\left(y,\left(X_{1}^{c}\right)^{\sim 2}\right)\right) 3 B_{3}^{2} \delta_{1} .
\end{aligned}
$$


This inequality and the inequalities (2.50) yield finally

$$
\begin{aligned}
& \left|\delta \phi_{k}(s)\right|<B_{3}^{2} \delta_{1}, \\
& \quad\left|\chi_{\Delta(y)}\left(\delta \phi_{k}(s)-\delta \phi_{k}(1)\right)\right|<\exp \left(-\frac{1}{4} \gamma_{0} d\left(y,\left(X_{1}^{c}\right)^{\sim 2}\right)\right) 4 B_{3}^{2} \delta_{1} .
\end{aligned}
$$

The function $\chi_{X_{0}} \delta \phi_{k}(s)$ has also a similar localization property as $\delta \alpha_{k}(s)$ : if $s=0$ on a family $\mathscr{C}$ of cubes, then $\chi_{X_{0}} \delta \phi_{k}(s)$ does not depend on $\phi_{0}, \alpha_{0}, \delta \psi, f_{1}, \alpha_{1}$ restricted to the domain $\bigcup_{\square \in \mathscr{C}} \square$. Actually, a stronger statement holds, the function depends on the variables restricted to this connected component of the domain $\left(\bigcup_{\square \in \mathscr{C}} \square\right)^{c}$ which contains $X_{0}$.

We summarize the main results obtained until now in this section in the following proposition.

Proposition 2.1. Consider the solutions $\delta \phi_{k}, \delta \alpha_{k}$ of the system of Eqs. (3.20) [3] defined on the space (3.21) [3]. There exist constants $B_{1}, K_{3}, c_{6}$ such that $B_{1} c_{6} \leqq \frac{1}{8}$, $c_{6} \leqq \gamma_{2}$, and if $B_{1} \delta_{0} \leqq \frac{1}{8}, B_{3} \delta_{1} \leqq \frac{1}{2} c_{6}$, then the solutions are extended to analytic functions $\delta \phi_{k}(s), \delta \alpha_{k}(s)$ defined on $\left\{s:|s| \leqq e^{\kappa_{1}}\right\}$. The function $\delta \alpha_{k}(s)$ satisfies Eq. (2.30), and $\delta \phi_{k}(s)$ is given by the formula (2.16) with $\delta \alpha=\delta \alpha_{k}(s)$. For $s=1$ they coincide with the solutions $\delta \phi_{k}, \delta \alpha_{k}$, i.e. $\delta \phi_{k}(1)=\delta \phi_{k}, \delta \alpha_{k}(1)=\delta \alpha_{k}$. Let $\mathscr{C}$ be a family of the large cubes, and let $s_{\square}=0$ for $\square \in \mathscr{C}$. Denote $Y_{0}=\bigcup_{\square \in \mathscr{C}} \square$, then $\chi_{X_{0}} \delta \phi_{k}(s)$ depends on the variables in the space (3.21) [3] restricted to the connected component of $Y_{0}^{c}$ containing the domain $X_{0}, \delta \alpha_{k}(s)=0$ on $Y_{0}$ and $\chi_{\Delta(y)} \delta \alpha_{k}(s)$ depends on the variables restricted to the component of $Y_{0}^{c}$ containing $\Delta(y)$. The functions $\delta \phi_{k}(s), \delta \alpha_{k}(s)$ satisfy the bounds

$$
\begin{gathered}
\left|\delta \phi_{k}(s)\right|<K_{3} \delta_{1}, \quad\left|\partial^{\eta} \delta \phi_{k}(s)\right|_{1}<K_{3} \delta_{1}, \\
\left|\partial^{\eta} \delta \phi_{k}(s)\right|_{(\alpha), 2-\alpha}<C_{\alpha} K_{3} \delta_{1} \quad \text { for } 0<\alpha \leqq 1, \\
\left|\Delta_{\Omega_{1}}^{D, \eta} \delta \phi_{k}(s)\right|_{2}<K_{3} \delta_{1},\left|\delta \alpha_{k}(s)\right|_{2}<K_{3} \delta_{1}, \\
\left|\chi_{\Delta(y)}\left(\delta \phi_{k}(s)-\delta \phi_{k}(1)\right)\right|<\exp \left(-\frac{1}{4} \gamma_{0} d\left(y,\left(X_{1}^{c}\right)^{\sim 2}\right)\right) K_{3} \delta_{1},
\end{gathered}
$$

and the same bounds for the remaining norms written in (2.54), only with the additional factor $C_{\alpha}$ for the third norm,

$$
\left|\chi_{\Delta(y)}\left(\delta \alpha_{k}(s)-\delta \alpha_{k}(1)\right)\right|_{2}<\exp \left(-\frac{1}{4} \gamma_{0} d\left(y,\left(X_{1}^{c}\right)^{\sim 2}\right)\right) K_{3} \delta_{1}
$$

It follows from (2.53), (2.45) and (2.49) that we can take $K_{3}=4 B_{3}^{2}$ in the above proposition. We can also simplify the formulation assuming $K_{3}=B_{1}$ without any important loss of generality, although $B_{1}$ is considerably bigger than $4 B_{3}^{2}$. The estimates of the two differences are particularly important in the future. We need them on the domain $X_{0}$, and it is enough then to replace the exponential functions of $y$ by small exponential factors. We use the bound (2.22), and we obtain the inequalities

$$
\left|\chi_{X_{0}}\left(\delta \phi_{k}(s)-\delta \phi_{k}\right)\right|<\exp \left(-\frac{1}{4} \gamma_{0} r_{1} M\right) K_{3} \delta_{1},
$$


and the corresponding bounds for the remaining norms in (2.54),

$$
\left|\chi_{X_{0}}\left(\delta \alpha_{k}(s)-\delta \alpha_{k}\right)\right|_{2}<\exp \left(-\frac{1}{4} \gamma_{0} r_{1} M\right) K_{3} \delta_{1},
$$

where $r_{1}=r_{0}-4$ is a positive integer, e.g. we may take $r_{1}=4$.

The above proposition is an extension of Proposition 3.1 [3], and it plays a fundamental technical role in all localization expansions we construct in these papers. Let us apply it now to the solution $\phi_{k}, \alpha_{k}$ of the basic system of Eqs. (1.14) [3]. This solution has been constructed in the form $\phi_{k}=\phi_{0}+\delta \phi_{k}, \alpha_{k}=\alpha_{0}+\delta \alpha_{k}$, where $\phi_{0}, \alpha_{0}$ is the approximate solution defined in (3.2), (3.3) [3], and the functions $\delta \phi_{k}, \delta \alpha_{k}$ satisfy the system (3.20) with the above $\phi_{0}, \alpha_{0}$, and with $\delta \psi=0, f_{1}=$ $-f_{0}, \alpha_{1}=0$. In this case $\delta_{0}=C_{3} \delta, \delta_{1}=C_{3} \delta$, where $\delta$ satisfies the assumptions of Proposition 2.1 [3], i.e., $0<\delta \leqq \varepsilon, \delta \leqq c_{4}, v \varepsilon^{2} \leqq \delta^{2}$. We assume that $B_{1} C_{3} \delta \leqq \frac{1}{8}$, $B_{3} C_{3} \delta \leqq \frac{1}{2} c_{6}$, which means that $\delta \leqq c_{7}$, where $c_{7}=\min \left\{c_{4}, \frac{1}{8}\left(B_{1} C_{3}\right)^{-1}\right.$, $\left.\frac{1}{2}\left(B_{3} C_{3}\right)^{-1} c_{6}\right\}$. Then the solutions $\delta \phi_{k}, \delta \alpha_{k}$ can be extended to the analytic functions $\delta \phi_{k}(s), \delta \alpha_{k}(s)$ by Proposition 2.1, with one small modification. In the above constructions we have used the localized Green's functions $G\left(\square_{z}, \alpha\right), \alpha=\alpha_{0}$ or $\alpha=\alpha_{0}+\delta \alpha$ and we have obtained the localization property with respect to $\alpha_{0}$. It is not good enough now, because we are interested in localization with respect to the variables $\left(\psi+\psi^{\prime}, h+h^{\prime}\right)$, and $\chi_{\Delta(y)} \alpha_{0}$ depends on these variables restricted to $\tilde{\Delta}(y)$, or even to $\tilde{\Delta}\left(y^{\prime}\right)$, where $y^{\prime}$ is a center of a block containing the point $y$. For $\Delta(y)$ close to the boundary of $\square_{z}$ this will involve a dependence of $G\left(\square_{z}, \alpha_{0}\right)$ on $\left(\psi+\psi^{\prime}, h+h^{\prime}\right)$ beyond the cube $\square_{z}$, on some neighborhood of $\square_{z}$. It is a small neighborhood, but to have the same simple formulation as in Proposition 2.1 we modify slightly the Green's functions. We use the fact that $\operatorname{supp} h_{z} \subset \square_{z}^{0}$, where $\square_{z}^{0}$ is a cube with a center at $z$ and of the size $2 M L^{j} \eta$, if $z \in \Lambda_{j}^{(m)+}$. From this it follows that $\alpha_{0} h_{z}=\chi_{\square_{z}^{0}} \alpha_{0} h_{z}$, hence we can replace the Green's functions $G\left(\square_{z}, \alpha\right)$ by $G\left(\square_{z}, \chi_{\square_{z}^{0}} \alpha\right)$, and all the formulas, bounds and statements remain the same. Now the function $\chi_{\square_{z}^{0}} \alpha_{0}$ depends on $\left(\psi+\psi^{\prime}, h+h^{\prime}\right)$ restricted to the small neighborhood of $\square_{z}^{0}$, which is contained in $\square_{z}$. Therefore the localization property with respect to these variables holds in the same form as in Proposition 2.1. Thus we define

$$
\phi_{k}(s)=\phi_{0}+\delta \phi_{k}(s), \quad \alpha_{k}(s)=\alpha_{0}+\delta \alpha_{k}(s),
$$

and we obtain analytic extensions of the solutions $\phi_{k}, \alpha_{k}$. They do not satisfy Eqs. (1.14) [3], in particular the relation $\alpha=\frac{\lambda}{2}\left(\phi^{2}-1\right)$ does not hold for them. Otherwise they satisfy all the properties formulated in Proposition 1.1 [3], and also the remaining ones in Proposition 2.1, with the constant $K_{4}=\left(2 K_{3}+1\right) C_{3}$. We formulate these conclusions in the following proposition.

Proposition 2.2. Consider the solutions of Eqs. (1.14) [3] with the assumptions of Proposition 1.1 [3]. There exist positive constants $c_{7}, K_{4}$ such that if $\delta \leqq c_{7}$ then the solutions have the analytic extensions $\phi_{k}(s), \alpha_{k}(s)$ defined by (2.58). They satisfy the bounds (1.15), (1.16), of Proposition 1.1 [3] with the constant $K_{4}$ instead of $K_{1}$, and the differences $\phi_{k}(s)-\phi_{k}, \alpha_{k}(s)-\alpha_{k}$ satisfy the bounds (1.15) [3], except the first and the sixth ones, on the domain $\Omega_{j} \cap X_{0}$ with $K_{1}$ replaced by $\exp \left(-\frac{1}{4} \gamma_{0} d\left(y,\left(X_{1}^{c}\right)^{\sim 2}\right)\right) K_{4}$, or by $\exp \left(-\frac{1}{4} \gamma_{0} r_{1} M\right) K_{4}$. If $s=0$ on the family of cubes $\mathscr{C}$, as in Proposition 2.1, then $\chi_{X_{0}} \phi_{k}(s), \chi_{X_{0}} \alpha_{k}(s)$ depend on the variables $\left(\psi+\psi^{\prime}, h+h^{\prime}\right)$ restricted to the component of $Y_{0}^{c}$ containing $X_{0}$. 
The existence and the properties of the analytic extensions $\phi_{k}(s), \alpha_{k}(s)$ imply existence and properties of $\psi_{k}^{(j)}(s)$, where $\psi_{k}^{(j)}$ is the simplified notation for the function $\psi\left(\mathbb{B}_{j}, \mathbb{B}_{k}^{\prime}\right)$ constructed in Sect. 4 [3]. It is expressed by the formula (4.21) [3] in terms of the function $\phi_{k}^{\prime}=\phi\left(\mathbb{B}_{k}^{\prime}\right)$, and the extension of the last defines an extension of $\psi_{k}^{(j)}$ by that formula. We have also

$$
\begin{aligned}
\psi_{k}^{(j)}(s)-\psi_{k}^{(j)}= & Q\left(\mathbb{B}_{j}\right)\left(\delta \phi_{k}^{\prime}(s)-\delta \phi_{k}^{\prime}\right) \\
& -\frac{1}{a\left(\mathbb{B}_{j}\right)} Q^{*}\left(\mathbb{B}_{k}^{\prime}, \mathbb{B}_{j}\right) a\left(\mathbb{B}_{k}^{\prime}\right) Q\left(\mathbb{B}_{k}^{\prime}\right)\left(\delta \phi_{k}^{\prime}(s)-\delta \phi_{k}^{\prime}\right),
\end{aligned}
$$

or more explicitly, for $y \in \Delta_{p}\left(y^{\prime}\right) \cap \Lambda_{n}, y^{\prime} \in \Lambda_{p}^{\prime}$,

$$
\begin{aligned}
\psi_{k}^{(j)}(y ; s)-\psi_{k}^{(j)}(y)= & Q_{n}\left(\delta \phi_{k}^{\prime}(s)-\delta \phi_{k}^{\prime}\right)(y) \\
& -\frac{a_{p}}{a_{n}}\left(L^{n} L^{-p}\right)^{2} Q_{p}\left(\delta \phi_{k}^{\prime}(s)-\delta \phi_{k}^{\prime}\right)\left(y^{\prime}\right) .
\end{aligned}
$$

This representation implies that the difference $\psi_{k}^{(j)}(s)-\psi_{k}^{(j)}$ satisfies the bound (2.55), or (2.57) on $X_{0} \cap \mathbb{B}_{j}$. We will need to know that $\psi_{k}^{(j)}(s)$ satisfies also the properties (4.29), (4.37) [3]. Let us consider again the proofs of these statements. In those proofs we have used the bounds (1.15),(1.16) [3] only, with one exception. Estimating the expression $\left|\psi_{k}^{(j)}(y)\right|-1$ we have used the second equation (1.14) [3]. This equation is not satisfied by the analytically extended configurations $\phi_{k}^{\prime}(s), \alpha_{k}^{\prime}(s)$, so we have to use instead an almost identical argument relying on properties of $\phi_{0}^{\prime}, \alpha_{0}^{\prime}$. We have

$$
\begin{aligned}
|| \psi_{k}^{(j)}(y ; s)|-1| & <||\left(Q_{n} \phi_{k}^{\prime}(s)\right)(y)|-1|+\left(L^{n} L^{-p}\right)^{2} K_{4} \delta<||\left(Q_{n} \phi_{0}^{\prime}\right)(y)|-1| \\
& +K_{3} C_{3} \delta+K_{4} \delta<\left.||\left(Q_{n} \phi_{0}^{\prime}\right)(y)\right|^{2}-1 \mid+\frac{3}{2} K_{4} \delta \leqq \frac{1}{2} \sum_{x_{1}, x_{2} \in B_{n}(y)} L^{-2 n d} \\
& \left|\phi_{0}^{\prime}\left(x_{1}\right)-\phi_{0}^{\prime}\left(x_{2}\right)\right|^{2}+\left(Q_{n} \|\left.\phi_{0}^{\prime}\right|^{2}-1 \mid\right)(y)+\frac{3}{2} K_{4} \delta \\
& <\frac{1}{2} d^{2}\left(L^{n} L^{-p}\right)^{2} C_{3}^{2} \delta^{2}+\frac{2}{\lambda}\left(Q_{n}\left|\alpha_{0}^{\prime}\right|\right)(y)+\frac{3}{2} K_{4} \delta \\
& <\frac{1}{2} d^{2} C_{3}^{2} \delta^{2}+\frac{2}{\lambda}\left(L^{p} \eta\right)^{-2} C_{3} \delta+\frac{3}{2} K_{4} \delta<2 K_{4} \delta,
\end{aligned}
$$

which is the same bound as in the proof of (4.29) [3], but with $K_{4}$ instead of $K_{1}$. Let us explain that in the above inequalities we take a real configuration $\left(\psi^{\prime}, h\right)$, but an arbitrary complex $s$ from the considered domain. The remaining arguments in the proofs of (4.29), (4.37) [3] are the same for $\psi_{k}^{(j)}(s)$, except that we get the constant $K_{4}$ instead of $K_{1}$. This yields the following proposition.

Proposition 2.3. The function $\psi\left(\mathbb{B}_{j}, \mathbb{B}_{k}^{\prime}\right)$ defined on a space $\tilde{\Psi}^{c}\left(\mathbb{B}_{k}^{\prime} ; \delta, \varepsilon\right)$ with $\delta, \varepsilon$ satisfying the assumptions of Proposition 1.1 [3] and $\delta \leqq c_{7}$ has the analytic extension $\psi\left(\mathbb{B}_{j}, \mathbb{B}_{k}^{\prime} ; s\right)$ defined in terms of $\phi\left(\mathbb{B}_{k}^{\prime} ; s\right)$ by the formula (4.21) [3]. It 
satisfies the properties (4.29), (4.37) [3], with the constant $K_{4}$ instead of $K_{1}$,

$$
\begin{aligned}
\left|\psi\left(\mathbb{B}_{j}, \mathbb{B}_{k}^{\prime} ; y ; s\right)-\psi\left(\mathbb{B}_{j}, \mathbb{B}_{k}^{\prime} ; y\right)\right| & <\exp \left(-\frac{1}{4} \gamma_{0} d\left(y,\left(X_{1}^{c}\right)^{\sim 2}\right)\right) K_{4} \delta \\
& <\exp \left(-\frac{1}{4} \gamma_{0} r_{1} M\right) K_{4} \delta \text { on } X_{0} \cap \mathbb{B}_{j},
\end{aligned}
$$

and the same localization property as in Proposition 2.2.

In the following sections we have to use the extensions for background functions determined by a generating set $\mathbb{B}_{k+1}$. They usually involve also the set $\mathbb{B}_{k}$ obtained by omitting the $k+1^{\text {st }}$ domain $\Omega_{k+1}$, and we consider them on lattices connected with the basic lattice in the $\eta$-scale, i.e., with $T_{\eta}$. We construct their extensions taking the partition into large cubes determined by $\mathbb{B}_{k}$ only, that is disregarding the domain $\Omega_{k+1}$. For cubes $\square_{z}$ intersecting $\Omega_{k+1}$ we take the corresponding operators $G\left(\square_{z}, \alpha\right)$ as in (2.3), but with the operator $a L^{-2} Q_{k+1}^{*} Q_{k+1}$ on the intersection $\square_{z} \cap$ $\Omega_{k+1}$. These operators satisfy the same basic bounds $(2.5),(2.7),(2.10)$ with properly chosen constants $B_{0}, \gamma_{0}$, hence all the remaining bounds, and Propositions 2.1-2.3 hold for such background functions also.

\section{Localization Expansions for Functions of the Covariance Operator}

Besides the background configurations in the function (1.1) we have the operator $C_{\Lambda}^{(k) \frac{1}{2}}$, which depends non-locally on $\theta$ through the configurations $\phi_{k+1}=$ $\phi\left(\mathbb{B}_{k+1}\right), \alpha_{k+1}=\alpha\left(\mathbb{B}_{k+1}\right)$. It is a function of an operator defining a basic quadratic form in $k+1^{\text {st }}$ fluctuation integrals. We will discuss these integrals in the next paper, where we will derive the following formula for the quadratic form:

$$
\begin{aligned}
& \left\langle\psi^{\prime},\left(a L^{-2} Q^{*} Q+\Delta^{(k)}\right) \psi^{\prime}\right\rangle \\
& =a L^{-2}\left\|Q \psi^{\prime}\right\|_{L}^{2}+a_{k}\left\|\psi^{\prime}\right\|^{2}-a_{k}^{2}\left\langle\psi^{\prime}, Q_{k} G\left(\alpha_{k+1}\right) Q_{k}^{*} \psi^{\prime}\right\rangle \\
& \quad+a_{k}^{2}\left\langle\phi_{k+1} \cdot G\left(\alpha_{k+1}\right) Q_{k}^{*} \psi^{\prime},\left[\frac{1}{\lambda_{k}}+\phi_{k+1} \cdot G\left(\alpha_{k+1}\right) \phi_{k+1}\right]^{-1} \phi_{k+1}\right. \\
& \left.\quad \cdot G\left(\alpha_{k+1}\right) Q_{k}^{*} \psi^{\prime}\right\rangle .
\end{aligned}
$$

Here the operator $G(\alpha)$ is determined by the generating set $\mathbb{B}_{k}$ and defined by (3.22) [2], and configurations $\psi^{\prime}$ have supports in $\Lambda=\Omega_{k+1}^{(k)} \subset T_{1}^{(k)}$. It is rather easy to see that for real configurations $\phi_{k+1}, \alpha_{k+1}$ the operator of the above quadratic form is symmetric, positive and bounded and we will prove it in the next paper. We are interested in two functions of this operator, namely in

$$
C_{\Lambda}^{(k) \frac{1}{2}}=\left(\left(a L^{-2} Q^{*} Q+\Delta^{(k)}\right) \uparrow_{\Lambda}\right)^{-\frac{1}{2}} \text { and } \log \left(\left(a L^{-2} Q^{*} Q+\Delta^{(k)}\right) \uparrow_{\Lambda}\right)
$$


The second will appear as a main contribution to logarithm of the fluctuation integral. We consider these functions for the operator with the background configurations $\phi_{k+1}, \alpha_{k+1}$ replaced by arbitrary configurations $\phi_{0}, \alpha_{0}$ in the space (3.21) of [2], with $\delta_{0}$ sufficiently small. Because of their importance let us write here the conditions defining the space

$$
\frac{1}{2}<\left|\phi_{0}\right|<\frac{3}{2},\left|\phi_{0}^{2}-1\right|<\delta_{0},\left|\partial^{\eta} \phi_{0}\right|_{1}<\delta_{0},\left|\Delta^{\eta} \phi_{0}\right|_{2}<\delta_{0}, \quad\left|\alpha_{0}\right|_{2}<\delta_{0} .
$$

At the moment we consider real configurations, but in the future we will extend all constructions onto the complex space. The operator $a L^{-2} Q^{*} Q+\Delta^{(k)}$ defined by configurations $\phi_{0}, \alpha_{0}$ and restricted to the set $\Lambda$ is symmetric, positive and bounded. Denote a positive lower bound by $\gamma_{0}$. We can define a function $f$ of this operator, where $f(z)$ is an analytic function on a domain containing the interval $\left[\gamma_{0},+\infty\right]$, by the formula

$$
f\left(a L^{-2} Q^{*} Q+\Delta^{(k)}\right)=\frac{1}{2 \pi i} \int_{C} d z f(z)\left(z I-\left(a L^{-2} Q^{*} Q+\Delta^{(k)}\right)\right)^{-1},
$$

where $C$ is a contour in the analyticity domain of $f(z)$ and surrounding the spectrum of the operator. For simplicity we omit the set $\Lambda$ in all notations; we assume that all the unit lattice operators are restricted to $\Lambda$ without writing it explicitly. We are interested in two functions: one is $f(z)=z^{-\frac{1}{2}}=\frac{1}{\sqrt{z}}$, more precisely we take a branch of $\sqrt{z}$ having positive values for $z$ real positive, and a cut along the real negative half-axis; another is $f(z)=\log z$, which is a branch of logarithm having real values for $z$ real positive, and a cut along the real negative half-axis. By the formula (3.3) the localization problems for the two operators are reduced to such a problem for the resolvent of the basic operator $a L^{-2} Q^{*} Q+\Delta^{(k)}$. We solve it by constructing a generalized random walk expansion for the resolvent, similar to the expansions $(2.8),(2.13)$. There are at least two ways of constructing such an expansion. We can do it using the method and the theorem on unit lattice operators in the fifth section of [2a]. This can be applied directly to the resolvent operator, but it requires an additional construction of a random walk expansion for the operator $\Delta^{(k)}$. It turns out that it is possible to obtain an explicit representation of the resolvent, which is quite similar to the formula (3.1), and we can construct a random walk expansion based on it. This is a bit shorter way, and we follow it here. To simplify properties of a representation of the resolvent we transform the integral in the formula (3.3), taking into account the particular functions we are interested in. The resolvent itself is analytic on the whole plane, except an interval $\left[\gamma_{0}, \gamma_{1}\right]$ in the positive half-axis. For the functions described above we can take the contour $C$ as a union of a circle $|z|=R$ with a large radius $R$, a circle $|z|=r$ with a small radius $r$, and two "sides" of the interval $[-R,-r]$. For the function $f(z)=z^{-\frac{1}{2}}$ we can take the limits $R \rightarrow+\infty, r \rightarrow 0+$ in the integral, and we obtain the representation

$$
C^{(k) \frac{1}{2}}=\frac{1}{\pi} \int_{0}^{+\infty} \frac{d x}{\sqrt{x}}\left(a L^{-2} Q^{*} Q+\Delta^{(k)}+x I\right)^{-1} .
$$

For the function $f(z)=\log z$ it is slightly more complicated, because $|\log z| \rightarrow+\infty$ as $|z| \rightarrow+\infty$, so we cannot take the limit $R \rightarrow+\infty$. In this case we consider 
$[-R,-r]$ as a union of two subintervals $\left[-R,-R_{0}\right]$ and $\left[-R_{0},-r\right]$, and we write the integral (3.3) as a sum of two integrals, one given by a contour which is a union of the large circle and two "sides" of the interval $\left[-R,-R_{0}\right]$, another given by a contour which is a union of the small circle and two "sides" of the interval $\left[-R_{0},-r\right]$. For the first integral we use the resolvent equation, which yields the additional factor $\frac{1}{2}$, and then we take the limit $R \rightarrow+\infty$. For the second integral we can take the limit $r \rightarrow 0+$. We obtain the following representation:

$$
\begin{aligned}
\log \left(a L^{-2} Q^{*} Q+\Delta^{(k)}\right)= & \left(a L^{-2} Q^{*} Q+\Delta^{(k)}\right) \int_{R_{0}}^{+\infty} \frac{d x}{x}\left(a L^{-2} Q^{*} Q+\Delta^{(k)}+x I\right)^{-1} \\
& -\int_{0}^{R_{0}} d x\left(a L^{-2} Q^{*} Q+\Delta^{(k)}+x I\right)^{-1}+\log R_{0}
\end{aligned}
$$

It is easy to see directly, using the resolvent equation, that the right-hand side above does not depend on $R_{0}$. We will use it in the future. To derive the representation of the resolvent in the formulas (3.4),(3.5) we start with the formula

$$
\begin{aligned}
\exp \left[\frac{1}{2}\left\langle f, C_{x}^{(k)} f\right\rangle\right] & =\frac{1}{Z_{x}^{(k)}} \int d \psi \exp \left[-\frac{1}{2} a L^{-2}\|Q \psi\|_{L}^{2}-\frac{1}{2}\left\langle\psi, \Delta^{(k)} \psi\right\rangle\right. \\
& \left.-\frac{1}{2} x\|\psi\|^{2}+\langle\psi, f\rangle\right], \\
C_{x}^{(k)} & =\left(a L^{-2} Q^{*} Q+\Delta^{(k)}+x I\right)^{-1},
\end{aligned}
$$

where $f$ is an arbitrary function on $\Lambda$ with values in $\mathbb{R}^{N}$, and we take $x \geqq 0$. Let us recall again that the variable $\psi$ in (3.6) is restricted to $\Lambda$. Consider the exponential factor $\exp \left[-\frac{1}{2}\left\langle\psi, \Delta^{(k)} \psi\right\rangle\right]$ under the integral. It can be represented by the integrals

$$
\begin{aligned}
\exp \left[-\frac{1}{2}\left\langle\psi, \Delta^{(k)} \psi\right\rangle\right]= & \frac{1}{Z_{k}} \int d \phi \exp \left[-\frac{1}{2}\langle\psi-Q \phi, a(\psi-Q \phi)\rangle\right. \\
& \left.-\frac{1}{2}\left\langle\phi,\left(-\Delta^{\eta}+v_{k}+\alpha_{0}\right) \phi\right\rangle-\frac{1}{2} \lambda_{k}\left\|\phi_{0} \cdot \phi\right\|^{2}\right] \\
= & \frac{1}{Z_{k}^{\prime}} \int d \phi \int d \alpha \exp \left[-\frac{1}{2}\langle\psi-Q \phi, a(\psi-Q \phi)\rangle\right. \\
& -\frac{1}{2}\left\langle\phi,\left(-\Delta^{\eta}+v_{k}+\alpha_{0}\right) \phi\right\rangle-i\left\langle\alpha, \phi_{0} \cdot \phi\right\rangle \\
& \left.-\frac{1}{2 \lambda_{k}}\|\alpha\|^{2}\right]
\end{aligned}
$$

where the variables $\phi, \alpha$ are restricted to the domain $\Omega_{1}$, the first domain in the sequence connected with the generating set $\mathbb{B}_{k}$, and $Q$ denotes the corresponding averaging operation $Q\left(\mathbb{B}_{k}\right)$, as in (3.22) [3]. Notice that $Q$ in the formulas (3.1), (3.3)-(3.6) denotes $Q_{1}$, i.e., the basic 1-block averaging operation. It is easy to see that integrating first with respect to $\phi$, and then with respect to $\alpha$, yields the corresponding quadratic form on the right-hand side of (3.1), which represents $\Delta^{(k)}$. Now we substitute the last integral above in place of the exponential factor in 
the integral (3.6), and we obtain a Gaussian integral in the variables $\psi, \phi, \alpha$. We integrate first with respect to $\psi$, then with respect to $\phi$, and finally with respect to $\alpha$. We do not discuss here these elementary Gaussian integrations. They are reduced to a simple linear algebra; let us write a final result only. We introduce the following two operators:

$$
\begin{aligned}
G_{k, x}\left(\alpha_{0}\right)= & \left(-\Delta_{\Omega_{1}}^{D, \eta}+a_{k} Q_{k}^{*}\left[\frac{x}{a_{k}+x}\left(I-Q^{*} Q\right)+\frac{a L^{-2}+x}{a_{k}+a L^{-2}+x} Q^{*} Q\right] Q_{k}\left\lceil\Omega_{k+1}\right.\right. \\
& +Q^{*}\left(\mathbb{B}_{k}\right) a\left(\mathbb{B}_{k}\right) Q\left(\mathbb{B}_{k}\right)\left\lceil\Omega_{k+1}^{c}+v_{k}+\alpha_{0}\right)^{-1} \\
A_{k, x}= & \frac{1}{a_{k}+x}\left(I-Q^{*} Q\right)+\frac{1}{a_{k}+a L^{-2}+x} Q^{*} Q
\end{aligned}
$$

where, in order to avoid confusion, we have written explicitly which operators are determined by $\mathbb{B}_{k}$. The operator $C_{x}^{(k)}$ has the representation

$$
\begin{gathered}
C_{x}^{(k)}=A_{k, x}+a_{k}^{2} A_{k, x} Q_{k} G_{k, x}\left(\alpha_{0}\right) Q_{k}^{*} A_{k, x}-a_{k}^{2} A_{k, x} Q_{k} G_{k, x}\left(\alpha_{0}\right) \phi_{0} \\
\otimes\left[\frac{1}{\lambda_{k}}+\phi_{0} \cdot G_{k, x}\left(\alpha_{0}\right) \phi_{0}\right]^{-1} \phi_{0} G_{k, x}\left(\alpha_{0}\right) Q_{k}^{*} A_{k, x} .
\end{gathered}
$$

It is very similar to the representation (3.1) for $\Delta^{(k)}$, the most important change is that the operator $G\left(\alpha_{0}\right)$ in (3.1) is replaced by $G_{k, x}\left(\alpha_{0}\right)$ given by (3.8). Let us notice that the above representation holds quite generally for the resolvent operator, and we have the identity (3.9) with $x$ replaced by $-z$, for $z$ in a quite large domain in the complex plane, but then we have more complicated properties and bounds of the operator $G_{k,-z}\left(\alpha_{0}\right)$. They become very simple for $-z=x \geqq 0$. The second operator in the parenthesis in (3.8) is equal to the linear combination of two projection operators

$$
\begin{aligned}
& \frac{a_{k} x}{a_{k}+x} Q_{k}^{*} Q_{k}+\frac{a_{k}^{2} a L^{-2}}{\left(a_{k}+x\right)\left(a_{k}+a L^{-2}+x\right)} Q_{k+1}^{*} Q_{k+1} \\
& \geqq \frac{3}{4} a_{k+1} L^{-2} Q_{k+1}^{*} Q_{k+1} \quad \text { for } 0 \leqq x \leqq \frac{1}{8} a_{k}, \quad \text { and } \\
& \geqq \frac{1}{9} a_{k} Q_{k}^{*} Q_{k} \quad \text { for } x>\frac{1}{8} a_{k} .
\end{aligned}
$$

From the above inequalities we conclude easily that the operator in the parentheses in (3.8), i.e., the operator $G_{k, x}^{-1}\left(\alpha_{0}\right)$, is positive for $\alpha_{0}$ real and sufficiently small, or its real part is positive for $\alpha_{0}$ complex and sufficiently small. We can get a more precise bound using the following well known simple inequalities:

$c\left(-\Delta_{\Delta}^{\eta, N}\right)+c^{\prime} Q_{j}^{*} Q_{j} \geqq\left(L^{j} \eta\right)^{-2} \min \left\{4 c, c^{\prime}\right\}$ on $L^{2}(\Delta)$, where $\Delta$ is a $j$-block, $j \leqq k$ and $\Delta_{\Delta}^{\eta, N}$ is the Laplace operator on $\Delta$ with Neumann boundary conditions; $c\left(-\Delta_{\Delta^{\prime}}^{\eta, N}\right)+c^{\prime} L^{-2} Q_{k+1}^{*} Q_{k+1} \geqq L^{-2} \min \left\{4 c, c^{\prime}\right\}$ on $L^{2}\left(\Delta^{\prime}\right)$, where $\Delta^{\prime}$ is a $k+1$-block, i.e., an $L$-cube of $T_{\eta}$. 
Of course we have assumed that $c, c^{\prime}$ are positive numbers. Let us take a cube $\square \subset \Omega_{k}$ which is a union of $k+1$-blocks. By quite elementary considerations, using the above inequalities, we obtain the bound

$$
\begin{gathered}
\operatorname{Re}\left[e ( q ) ^ { - 1 } \left(-\Delta_{\square}^{\eta, N}+\left[\frac{a_{k} x}{a_{k}+x} Q_{k}^{*} Q_{k}+\frac{a_{k}^{2} a}{\left(a_{k}+x\right)\left(a_{k}+a L^{-2}+x\right)} L^{-2} Q_{k+1}^{*} Q_{k+1}\right]\left\lceil\cap \cap \Omega_{k+1}\right.\right.\right. \\
\left.\left.\quad+a_{k} Q_{k}^{*} Q_{k} \operatorname{\lceil \cap } \Omega_{k+1}^{c}+v_{k}+\alpha_{0}\right) e(q)\right] \geqq \frac{1}{3}\left(-\Delta_{\square}^{\eta, N}\right)+\frac{1}{6} a_{k+1} L^{-2}
\end{gathered}
$$

on $L^{2}(\square), \quad$ where $e\left(q ; x^{\prime}\right)=e^{q \cdot x^{\prime}}, \quad x^{\prime} \in T_{\eta}, q \in \mathbb{R}^{d}$

$$
\text { and }|q|_{\infty}=\max _{\mu}\left|q_{\mu}\right| \leqq \frac{1}{5 d} L^{-2}, \quad\left|\alpha_{0}\right|<\frac{1}{12} a_{k+1} L^{-2}, \quad x \geqq 0 \text {. }
$$

The numerical factors above are to some extent arbitrary. If we take a cube $\square \subset \Omega_{j}$, $j<k$, which may intersect $\Omega_{j+1}$, but does not intersect $\Omega_{j+2}$, and which is a union of $j+1$-blocks, then the operator in the parentheses in (3.8) restricted to this cube is equal to the corresponding operator in (2.3), and for it we have the above bound with the second term on the right-hand side multiplied by $\left(L^{j} \eta\right)^{-2}$. Let us define an operator $G_{k, x}\left(\square, \alpha_{0}\right)$ by the formula (3.8), but with the operator in the parentheses restricted to a cube $\square$, the Laplace operator with Neumann boundary conditions on $\square$. Of course we have $G_{k, x}\left(\square, \alpha_{0}\right)=G\left(\square, \alpha_{0}\right)$ if $\square$ is disjoint with $\Omega_{k+1}$. The operators $G_{k, x}\left(\square_{z}, \alpha_{0}\right)$ play a crucial role in constructing the generalized random walk expansion (2.8) for the operator $G_{k, x}\left(\alpha_{0}\right)$. Convergence of this expansion depends on the bounds (2.5). They hold for the operators $G_{k, x}\left(\square, \alpha_{0}\right)$ also, but strictly speaking the proofs given in [2a] do not cover this case, although they can be easily extended to yield the bounds. Instead of doing this we notice that now it is enough to prove the convergence in the $L^{2}$-norms, because we want to use the expansions for the operators in the expression on the right-hand side of (3.9), and the averaging operations $Q_{k}$ can be interpreted as taking scalar products with characteristic functions of $k$-blocks, that is with functions having $L^{2}$-norms equal to 1 . This is a crucial remark, because the bounds $(2.5)$ in $L^{2}$-norms are immediate consequences of the bound (3.10). We obtain

$$
\begin{aligned}
& L^{j} \eta\left\|\chi_{\Delta(y)} \partial^{\eta} G_{k, x}\left(\square, \alpha_{0}\right) \chi_{\Delta\left(y^{\prime}\right)} f\right\|+\left\|\chi_{\Delta(y)} G_{k, x}\left(\square, \alpha_{0}\right) \chi_{\Delta\left(y^{\prime}\right)} f\right\| \\
& \quad \leqq B_{0} e^{-\gamma_{0} d\left(y, y^{\prime}\right)}\left(L^{j^{\prime}} \eta\right)^{2}\left\|\chi_{\Delta\left(y^{\prime}\right)} f\right\|,
\end{aligned}
$$

where $y \in \Lambda_{j} \cap \square, y^{\prime} \in \Lambda_{j^{\prime}} \cap \square, f$ is an arbitrary function on $\square$, and we can take $B_{0}=15 \frac{1}{a_{k+1}} L^{2}, \gamma_{0}=\frac{1}{5 d} L^{-2}$. This bound implies the convergence of the corresponding expansion (2.8) in the $L^{2}$-norms. This expansion yields an expansion of the second term in the representation (3.9). It can be written in the form

$$
\begin{gathered}
\sum_{z} a_{k}^{2} A_{k, x}<\chi_{\Delta(\cdot)}, h_{z_{0}} G_{k, x}\left(\square_{z_{0}}, \alpha_{0}\right) h_{z_{0}} K_{x}\left(h_{z_{1}}\right) G_{k, x}\left(\square_{z_{1}}, \alpha_{0}\right) h_{z_{1}} \\
\left.\cdot \ldots \cdot K_{x}\left(h_{z_{n}}\right) G_{k, x}\left(\square_{z_{n}}, \alpha_{0}\right) h_{z_{n}} \chi_{\Delta(} \cdot\right)_{k, x}
\end{gathered}
$$


where the sum is over all walks $z=\left(z_{0}, z_{1}, \ldots, z_{n}\right)$, and the operator $K_{x}(f)$ is given by the formula,

$$
\begin{aligned}
& K_{x}(f)=2 \nabla f \cdot \nabla+\Delta f-\left(\frac{a_{k} x}{a_{k}+x}\left[Q_{k}^{*} Q_{k}, f\right]+\frac{a_{k}^{2} a L^{-2}}{\left(a_{k}+x\right)\left(a_{k}+a L^{-2}+x\right)}\right. \\
& \left.\quad \times\left[Q_{k+1}^{*} Q_{k+1}, f\right]\right)\left\lceil\Omega_{k+1}-a_{k}\left[Q_{k}^{*} Q_{k}, f\right]\left\lceil\Omega_{k+1}^{c},\right.\right.
\end{aligned}
$$

if $\operatorname{supp} f$ intersects $\Omega_{k+1}$. If it does not, then it is given by the second formula in (2.6), but with $h_{z}$ replaced by $f$. Instead of the bound (2.10) we use now the bound

$$
\left(L^{j} \eta\right)^{2}\left\|\chi_{\Delta(y)} K_{x}\left(h_{z}\right) G_{k, x}\left(\square_{z}, \alpha_{0}\right) h_{z} \chi_{\Delta\left(y^{\prime}\right)} f\right\| \leqq 4 d M^{-1} B_{0} e^{-\gamma_{0} d\left(y, y^{\prime}\right)}\left(L^{j^{\prime}} \eta\right)^{2}\left\|\chi_{\Delta\left(y^{\prime}\right)} f\right\|
$$

following from (3.10), (3.11) and (3.13). It implies the convergence of (3.12) in exactly the same way as in Sect. 2 , in particular the bounds (5.11) hold without any changes. We would like to construct a similar expansion for the third term on the right-hand side of (3.9). It is slightly more complicated because of the inverse operator in the middle of the expression. We have analyzed this operator in [3], so we summarize necessary results obtained there in the equalities below

$$
\begin{aligned}
{\left[\frac{1}{\lambda_{k}}+\phi_{0} \cdot G_{k, x}\left(\alpha_{0}\right) \phi_{0}\right]^{-1} } \\
=\left[\frac{1}{\lambda_{k}} G_{k, x}^{-1}\left(\alpha_{0}\right)+G_{k, x}^{-1}\left(\alpha_{0}\right)\left(\phi_{0} \cdot G_{k, x}\left(\alpha_{0}\right) \phi_{0}\right)\right]^{-1} G_{k, x}^{-1}\left(\alpha_{0}\right) \\
=\left[\frac{1}{\lambda_{k}} G_{k, x}^{-1}(0)+1-K_{x}\left(\phi_{0}\right) \cdot G_{k, x}\left(\alpha_{0}\right) \phi_{0}+\left(\phi_{0}^{2}-1+\frac{\alpha_{0}}{\lambda_{k}}\right)\right]^{-1} \\
\quad \times G_{k, x}^{-1}\left(\alpha_{0}\right)=\left[1-\lambda_{k} G_{k, x}\left(\lambda_{k}\right) K_{x}\left(\phi_{0}\right) \cdot G_{k, x}\left(\alpha_{0}\right) \phi_{0}+\lambda_{k} G_{k, x}\left(\lambda_{k}\right)\right. \\
\\
\left.\quad\left(\phi_{0}^{2}-1+\frac{\alpha_{0}}{\lambda_{k}}\right)\right]^{-1} \lambda_{k} G_{k, x}\left(\lambda_{k}\right) G_{k, x}^{-1}\left(\alpha_{0}\right) .
\end{aligned}
$$

The operator $\lambda_{k} G_{k, x}\left(\lambda_{k}\right)$ has the Hilbert space norm $\leqq 1$, the operator $K_{x}\left(\phi_{0}\right)$. $G_{k, x}\left(\alpha_{0}\right) \phi_{0}$ has a small norm of the order $O\left(\delta_{0}\right)$, and $\left|\phi_{0}^{2}-1+\frac{\alpha_{0}}{\lambda_{k}}\right|<2 \delta_{0}$ by (3.2), hence the operator in the last square brackets above is a small perturbation of the identity operator, and its inverse can be represented by a convergent Neumann series, for $\delta_{0}$ sufficiently small. Thus the third term in (3.9) is given by the following series:

$$
\begin{aligned}
& \sum_{n=0}^{\infty} a_{k}^{2} A_{k, x}<\chi_{\Delta(\cdot)}, G_{k, x}\left(\alpha_{0}\right) \phi_{0} \otimes\left(\lambda_{k} G_{k, x}\left(\lambda_{k}\right) K_{x}\left(\phi_{0}\right) \cdot G_{k, x}\left(\alpha_{0}\right) \phi_{0}\right. \\
& \left.\quad-\lambda_{k} G_{k, x}\left(\lambda_{k}\right)\left(\phi_{0}^{2}-1+\frac{\alpha_{0}}{\lambda_{k}}\right)\right)^{n}\left(\lambda_{k} G_{k, x}\left(\lambda_{k}\right) K_{x}\left(\phi_{0}\right) G_{k, x}\left(\alpha_{0}\right)\right. \\
& \left.\quad-\lambda_{k} G_{k, x}\left(\lambda_{k}\right) \phi_{0}\right) \chi_{\Delta(\cdot)}>A_{k, x} .
\end{aligned}
$$


It is a convergent series, whose $n^{\text {th }}$ term is a product of at least $n$ small factors, so we can obtain easily various uniform bounds for its sum. Now we construct a random walk expansion of the sum by substituting in place of all the operators $G_{k, x}\left(\alpha_{0}\right), G_{k, x}\left(\lambda_{k}\right)$ their random walk expansions. We obtain an expansion of the general form (3.12), but in which the operators $K_{x}\left(h_{z}\right) G_{k, x}\left(\square_{z}, \alpha_{0}\right) h_{z}$ may be replaced by other possible operators. We describe this by introducing generalized random walks, with points $z$ replaced by pairs $(z, i)$, where the natural number $i$ indicates which operator we take. We combine this expansion with (3.12), and we obtain the following expansion of the sum of the second and third terms on the right-hand side of (3.9), without the operators $a_{k} A_{k, x}$

$$
D_{k, x}\left(y, y^{\prime}\right)=\sum_{\omega}\left\langle\chi_{\Delta(y)}, \prod_{m=0}^{|\omega|} G_{\omega_{m}}\left(h_{z_{m}}, \square_{z_{m}}\right) h_{z_{m}} \chi_{\Delta\left(y^{\prime}\right)}\right\rangle,
$$

where the sum is over random walks $\omega=\left(\omega_{0}, \omega_{1}, \ldots, \omega_{n}\right),|\omega|=n, \omega_{m}=\left(z_{m}, i_{m}\right)$, and an operator $G_{\omega}\left(h_{z}, \square_{z}\right)$ is one of the operators

$$
\begin{aligned}
& h_{z} G_{k, x}\left(\square_{z}, \alpha_{0}\right), h_{z} G_{k, x}\left(\square_{z}, \alpha_{0}\right) \phi_{0}, K_{x}\left(h_{z}\right) G_{k, x}\left(\square_{z}, \alpha_{0}\right), \\
& \quad K_{x}\left(h_{z}\right) G_{k, x}\left(\square_{z}, \alpha_{0}\right) \phi_{0}, \lambda_{k} h_{z} G_{k, x}\left(\square_{z}, \lambda_{k}\right), K_{x}\left(h_{z}\right) G_{k, x}\left(\square_{z}, \lambda_{k}\right), \\
& \quad K_{x}\left(\phi_{0}\right) h_{z} G_{k, x}\left(\square_{z}, \alpha_{0}\right), K_{x}\left(\phi_{0}\right) \cdot h_{z} G_{k, x}\left(\square_{z}, \alpha_{0}\right) \phi_{0}, \\
& \quad-\lambda_{k} h_{z} G_{k, x}\left(\square_{z}, \lambda_{k}\right)\left(\phi_{0}^{2}-1+\frac{\alpha_{0}}{\lambda_{k}}\right),-K_{x}\left(h_{z}\right) G_{k, x}\left(\square_{z}, \lambda_{k}\right)\left(\phi_{0}^{2}-1+\frac{\alpha_{0}}{\lambda_{k}}\right), \\
& \quad-K_{x}\left(h_{z}\right) G_{k, x}\left(\square_{z}, \lambda_{k}\right) \phi_{0},-\lambda_{k} h_{z} G_{k, x}\left(\square_{z}, \lambda_{k}\right) \phi_{0} .
\end{aligned}
$$

Thus the indices $i_{m}$ change from 1 to 12 , but obviously they do not change independently; there is a set of restrictions following from the structure of the expressions in (3.12), and in the series (3.15). They should be quite clear, so we do not need to formulate them explicitly here, but let us mention a few which will be used next in bounds. The first two operators in (3.17) appear only at the beginning of the product in (3.16), i.e. for $m=0$, and $i_{0}=1$ or 2. The last operator may appear only at the end of the product. After the fifth operator there is in some place in the product (3.16) either the seventh, eighth, or tenth operator, except when the fifth operator appears the last time in the product. From the inequalities (3.10), (3.11), (3.14) we obtain the following bounds:

$$
\begin{aligned}
& \left\|\chi_{\Delta(y)} G_{\omega}\left(h_{z}, \square_{z}\right) h_{z} \chi_{\Delta\left(y^{\prime}\right)} f\right\| \leqq\left\{1, \frac{3}{2}, 4 d M^{-1}, 6 d M^{-1}, 1,4 d M^{-1},\right. \\
& \left.(2 d+1) \delta_{0}, \frac{3}{2}(2 d+1) \delta_{0}, 2 \delta_{0}, 8 d M^{-1} \delta_{0}, 6 d M^{-1}, \frac{3}{2}\right\} B_{0} e^{-\gamma_{0} d\left(y, y^{\prime}\right)} \\
& \left(L^{j^{\prime}} \eta\right)^{2}\left\|\chi_{\Delta\left(y^{\prime}\right)} f\right\|,
\end{aligned}
$$

where the order of constants corresponds to the order of operators in (3.17), and we have to include the factors $\left(L^{j} \eta\right)^{2}$ in the norms for the operators involving $K_{x}$. The bound of the fifth operator is not small, but we can use the fact that it is followed by one of the operators described above, whose norms are of the order $0\left(\delta_{0}\right)$, and assign the factor $\delta_{0}^{\frac{1}{2}}$ to it. Now we can estimate the expansion (3.16) 
in the same way as the expansion (2.8), and we get the inequalities (2.11) with minor changes only, more precisely the numerical factor on the right-hand side of the third inequality is replaced by

$$
9 B_{0}^{2} c_{1}^{2}\left(d, \frac{1}{4} \gamma_{0}\right)(2 L+1)^{d}\left(48 d B_{0} c_{1}\left(d, \frac{1}{4} \gamma_{0}\right)(2 L+1)^{d} \max \left\{M^{-1}, \frac{2}{3} \delta_{0}^{\frac{1}{2}}\right\}\right)^{n-1} .
$$

The remaining considerations are the same as in Sect.2, we assume that the expression in the parentheses above is $\leqq 1$, and this yields the convergence of the expansion (3.16), and various bounds for the sum and certain partial sums. In particular the whole sum, hence the operator $D_{k, x}$ satisfies the bound

$$
\begin{aligned}
\left|\left(D_{k, x} f\right)(y)\right| & <9 B_{0}^{2} c_{1}^{2}\left(d, \frac{1}{4} \gamma_{0}\right)(2 L+1)^{d} \sum_{\omega: y \in \square_{z_{0}}} \frac{1}{8^{n}} \frac{1}{(2 L+1)^{d n}} e^{-\frac{1}{2} \gamma_{0} d(y ; \omega)}|f| \\
& \leqq B_{4}|f|, \quad \text { where } B_{4}=9 \cdot 2^{d+1} B_{0}^{2} c_{1}^{2}\left(d, \frac{1}{4} \gamma_{0}\right)(2 L+1)^{d} .
\end{aligned}
$$

In the above bounds we have used the fact that any $\omega_{m}$ in a generalized random walk $\omega$ can be followed by an $\omega_{m+1}$ with at most 4 different indices $i_{m+1}$, and this is the reason for the number 48 in (3.19), and $\frac{1}{8^{n}}$ in (3.20).

Using the bound (3.20), which is uniform in $x \geqq 0$, and the equality

$$
C_{k, x}=A_{k, x}+a_{k}^{2} A_{k, x} D_{k, x} A_{k, x}
$$

following from the definition of $D_{k, x}$, we can simplify the formula (3.5). At first notice that the first integral on the right-hand side of (3.5) converges to 0 as $R_{0} \rightarrow$ $+\infty$, because the under-integral expression is of the order $O\left(\frac{1}{x^{2}}\right)$. The operator $A_{k, x}$ is a very simple operator given by the formula (3.8), and it is of the order $O\left(\frac{1}{x}\right)$ for large $x$, so the second integral in (3.5) of the second term on the right-hand side of (3.21) is convergent as $R_{0} \rightarrow+\infty$. This integral of the first term on the righthand side can be calculated explicitly, and the result combined with the term $\log R_{0}$ in (3.5) is convergent to $\log a_{k}\left(I-Q^{*} Q\right)+\log \left(a_{k}+a L^{-2}\right) Q^{*} Q$ as $R_{0} \rightarrow+\infty$. We can do a similar calculation for (3.4), and we obtain the following final formulas for the operators in (3.4), (3.5):

$$
\begin{aligned}
& C^{(k) \frac{1}{2}}=\frac{1}{\sqrt{a_{k}}}\left(I-Q^{*} Q\right)+\frac{1}{\sqrt{a_{k}+a L^{-2}}} Q^{*} Q+\frac{a_{k}^{2}}{\pi} \int_{0}^{+\infty} \frac{d x}{\sqrt{x}} A_{k, x} D_{k, x} A_{k, x}, \\
& \log \left(a L^{-2} Q^{*} Q+\Delta^{(k)}\right)=\log a_{k}\left(I-Q^{*} Q\right)+\log \left(a_{k}+a L^{-2}\right) Q^{*} Q \text {. } \\
& -a_{k}^{2} \int_{0}^{+\infty} d x A_{k, x} D_{k, x} A_{k, x}
\end{aligned}
$$

The expansion (3.16) yields random walk expansions of these operators. We can use them now either to introduce the localization parameters $s$ into the operators, or to construct their localization expansions directly. The introduction of the localization parameters has been discussed thoroughly in Sect. 2. We use the expansion (3.16) and construct an analytically extended operator $D_{k, x}(s)$ by the formula (2.16). Using 
the bounds (3.20) and the basic inequality (2.21) we obtain

$$
\left|D_{k, x}(s) f\right| \leqq B_{4} \exp \left((7 L)^{d} \kappa_{1}\right)|f|
$$

The extension of $D_{k, x}$ defines an extension $C^{(k) \frac{1}{2}}(s)$ through the formula (3.22). It satisfies the bound

$$
\left|C^{(k) \frac{1}{2}}(s) f\right| \leqq\left(\frac{2}{\sqrt{a_{k}}}+\frac{a_{k}^{2}}{\pi} \int_{0}^{+\infty} \frac{d x}{\sqrt{x}}\left(\frac{1+L^{-2}}{a_{k}+x}\right)^{2} B_{4} \exp \left((7 L)^{d} \kappa_{1}\right)\right)|f|=B_{5}|f|
$$

following from (3.22), (3.23). Notice that the bounds $(3.23),(3,24)$ can be improved by omitting the factor $\exp \left((7 L)^{d} \kappa_{1}\right)$, as in $(2.23)$, if we restrict the supremum norms on the left-hand sides to the domain $X_{0}$. Let us recall that the extensions in $s$ are defined for a given pair of localization domains $X_{0}, X_{1}$ satisfying (2.14). The above results form an important part of the whole localization problem, and we summarize them in the proposition below.

Proposition 3.1. The operator $C^{(k) \frac{1}{2}}$ given by the formulas (3.4), (3.9), (3.6), (3.22), (3.16), has the localization extension constructed above, which is an analytic function of $\left(\phi_{0}, \alpha_{0}\right)$ on the domain (3.2) with $\delta_{0}$ small enough, and of the localization parameters $s$ in $\left\{s:|s|<e^{\kappa_{1}}\right\}$. This localization extension satisfies the bound (3.24), and the bound corresponding to (2.60) with $K_{4} \delta$ replaced by $B_{5}|f|$. It satisfies also the crucial localization property, which can be formulated in the following form: if $s=0$ on a family $\mathscr{C}$ of cubes, and if $Y_{1}$ is the connected component of $(\cup \mathscr{C})^{c}$ containing $X_{1}$, then $\chi_{Y_{1}} C^{(k) \frac{1}{2}}\left(\phi_{0}, \alpha_{0}, s\right) f$ depends on $\phi_{0}, \alpha_{0}, s, f$ restricted to the domain $Y_{1}$.

Obviously similar conclusions can be formulated for the second operator in (3.22). Actually we will not need this operator, only its trace which appears as a leading term in contributions to the effective action coming from the fluctuation integral. We will discuss these conclusions later, together with the remaining localization expansion.

\section{A Construction of the Localization Expansion (1.4)}

In this section we construct the expansion (1.4), and prove complete analyticity statements, using the results of the two preceding sections. Let us recall the following identities for the background configurations occurring in (1.1):

$$
\begin{aligned}
& \psi\left(\mathbb{B}_{j}, \mathbb{B}_{k} ; \psi\left(\mathbb{B}_{k}, \mathbb{B}_{k+1} ; \theta\right)\right)=\psi\left(\mathbb{B}_{j}, \mathbb{B}_{k+1} ; \theta\right), \text { or simply } \\
& \psi_{k}^{(j)}\left(\psi^{(k)}(\theta)\right)=\psi_{k+1}^{(j)}(\theta), \quad \text { where } \psi^{(k)}(\theta)=\psi_{k+1}^{(k)}(\theta)=\psi\left(\mathbb{B}_{k}, \mathbb{B}_{k+1} ; \theta\right), \\
& \psi_{k}^{(j)}\left(\psi^{(k)}(\theta)+\beta_{k}^{-\frac{1}{2}} C^{(k) \frac{1}{2}} \psi\right)=\psi_{k+1}^{(j)}(\theta)+\delta \psi_{k}^{(j)}\left(\beta_{k}^{-\frac{1}{2}} C^{(k) \frac{1}{2}} \psi\right),
\end{aligned}
$$

where the last function on the right-hand side above is determined by the background configurations $\phi_{k+1}(\theta), \alpha_{k+1}(\theta)$, and for simplicity of notations we have omitted the dependence on $\Lambda$. These identities are special cases of the identities (4.21), (3.49) [3]. It seems that the last identity and the results of the two previous sections 
provide a natural way to construct the expansion; we could introduce properly the localization parameters $s$ into the functions on the right-hand side, and to interpolate in $s$ between 0 and 1 in a well known way. This is formally correct and yields a localized expansion, but unfortunately the functions $\psi_{k+1}^{(j)}(s), \delta \psi_{k}^{(j)}(s)$ do not have all necessary properties needed in our method, for example, their values do not generally belong to the space $\Psi_{j}^{c}\left(X ; C_{0} L^{j} \eta, \varepsilon_{k}\right)$, which we need for the bounds obtained in [1]. This is so because the introduction of the localization parameters destroys their structure, their algebraic properties and the equations satisfied by them. To overcome these difficulties we have to use a more elaborate representation than the one above. This will be done in several steps.

Let us start with geometric definitions. We have the localization domain $X \in \mathscr{D}_{j}$, and we would like to assign to it a "smallest" in some sense localization domain $X_{1} \in \mathscr{D}_{k+1}$ containing it. Let us recall that in the considered scale, in which the basic lattice $T$ is in the $\eta$-scale, i.e., it is $T_{\eta}$, a domain from $\mathscr{D}_{j}$ is a connected union of $L M L^{j} \eta$-cubes from the cover $\pi_{j}^{\prime}$, which are unions of $L^{d}$ cubes of the size $M L^{j} \eta$ from the partition $\pi_{j}$. We define the domain $X_{1} \in \mathscr{D}_{k+1}$ in the following way:

$$
X_{1}=\text { a union of all } L^{2} M \text {-cubes from the cover } \pi_{k+1}^{\prime} \text {, which contain a }
$$

$$
L M L^{j} \eta \text { - cube from the cover } \pi_{j}^{\prime} \text { contained in } X \text {. }
$$

We define also a domain $X^{\prime}$ as a union of all large cubes from the partition $\pi_{k+1}$ which have non-empty intersections with $X$. For these domains we have the inclusions

$$
X_{1} \subset X^{\prime \sim L-1}, \quad X_{1} \supset X^{\prime \sim L-2},
$$

for example for $L=3$ we have $X_{1} \subset X^{\prime \sim 2}, X_{1} \supset X^{\prime \sim}$. We would like to keep the possibility of having this small value of $L$, and we would like also to define a domain $X_{0}$ containing $X$ and satisfying the condition (2.14). This is possible if we introduce two scales of large cubes, one smaller for the constructions and results of this paper, and another larger for the main renormalization group procedure. We take the smaller scale in such a way that all conditions related to constructions and properties of all the minimizing functions in this paper are satisfied. There are several conditions involving the size $M$ of the large cubes, for example the conditions $8 d B_{0} c_{1}\left(d, \frac{1}{8} \gamma_{0}\right) \cdot(2 L+1)^{d} M^{-\frac{1}{2}} \leqq 1, M \geqq 4(7 L)^{d} \kappa_{1} \frac{1}{\gamma_{0}}$ used in the proofs of Sect. 2 and we assume that $M_{1}$ is a number of the form $L^{m_{1}}$ satisfying all these conditions. Thus all the statements proven until now hold for the number $M_{1}$. The size $M$ of large cubes used in the renormalization group procedure, i.e. in the paper [1], is greater than $M_{1}$, and we will obtain some conditions on it. From now on we have to be particularly careful with the meaning of the operation " $\sim$." In this paper it generally means that the size $M_{1}$ of large cubes is used, except in the cases where we have domains arising in the renormalization group procedure and the size $M$ is used, like in (4.2), (4.3).

Let us consider again the localization domain $X$. We would like to construct a minimal in some sense sequence $\left\{\Omega_{1}^{\prime}, \ldots, \Omega_{k}^{\prime}\right\}$ of localization domains defined in terms of $M_{1}$, satisfying (1.1) [3] and such that $X \subset \Omega_{k}^{\prime}$. We define $\Omega_{k}^{\prime}$ in the same way as $X_{1}$ was defined, with $M_{1}$ replacing $M$, we take

$$
\Omega_{k}^{\prime}=\text { a union of } L M_{1} \text {-cubes from the cover } \pi_{k}^{\prime} \text { which contain }
$$

$$
\text { a } L M_{1} L^{j} \eta \text {-cube from the cover } \pi_{j}^{\prime} \text { contained in } X \text {. }
$$


Notice that the conditions (1.1) [3] are satisfied if $\Omega_{n+1}^{\sim R} \subset \Omega_{n}$, where $\Omega_{n+1}$ is considered as a domain in $\mathscr{D}_{n}$, and the operation " $\sim$ " is defined in terms of cubes of the partition $\pi_{n}$. Until now we have only very mild restrictions on $R$, the strongest one we may impose is connected with the conditions (2.14), and may be formulated as $\Omega_{n+1}^{\sim r_{0} L} \subset \Omega_{n}$, where we may even take $r_{0}=5$. Thus a next domain in the sequence must satisfy this condition only, so we consider $\Omega_{k}^{\prime}$ as a localization domain in $\mathscr{D}_{k-1}$ and we define

$$
\Omega_{k-1}^{\prime}=\Omega_{k}^{\prime \sim 5 L} \text {, where we add layers of } M_{1} L^{k-1} \eta=M_{1} L^{-1} \text {-cubes. }
$$

Generally, having defined $\Omega_{n+1}^{\prime}$ we consider it as a localization domain in $\mathscr{D}_{n}$ and we define

$$
\Omega_{n}^{\prime}=\Omega_{n+1}^{\prime \sim 5 L}, \text { where we add layers of } M_{1} L^{j} \eta \text {-cubes. }
$$

This way we construct the whole sequence $\left\{\Omega_{1}^{\prime}, \Omega_{2}^{\prime}, \ldots, \Omega_{k}^{\prime}\right\}$ and the corresponding generating set. They are determined by the domain $X$, so we denote them by $\left\{\Omega_{1}(X), \Omega_{2}(X), \ldots, \Omega_{k}(X)\right\}$ and $\mathbb{B}_{k}(X)$. Notice that they depend on $k$ also, the domains $\Omega_{j}(X)$ vary with $k$, according to the above definition. We define also a domain $X^{\prime \prime}$ in the same way as the domain $X^{\prime}$ before, namely as a union of $M_{1}$-cubes from $\pi_{k}$ which have non-empty intersections with $X$. Obviously we have

$$
X \subset X^{\prime \prime} \subset X^{\prime \prime} \sim L-2 \subset \Omega_{k}(X) \subset X^{\prime \prime} \sim L-1 \subset X^{\prime \sim L-1},
$$

where the operation " $\sim$ " is determined by the $M_{1}$-cubes. We define

$$
\begin{aligned}
& X_{0}=X^{\prime \sim 4 L}, \text { hence } \Omega_{1}(X) \subset X_{0}, \text { because } \\
& \qquad \begin{aligned}
(L-1) M_{1}+5 L M_{1} L^{-1}+\cdots+5 L M_{1} L^{-(k-1)}<(L-1) M_{1} \\
\quad+\frac{15}{2} M_{1}<4 L M_{1} .
\end{aligned}
\end{aligned}
$$

The domain $X_{0}$ is a localization domain from the corresponding family $\mathscr{D}_{k}$ defined in terms of $M_{1}$, and we consider $X_{1}$ also as a domain from this family. We require that the two domains $X_{0}, X_{1}$ satisfy the condition (2.14), i.e., $X_{0}^{\sim r_{0}} \subset X_{1}$, the operation " $\sim$ " is determined by the partition into $M_{1}$-cubes. By the definition of $X_{0}$ and (4.3) this condition is satisfied if $4 L M_{1}+r_{0} M_{1} \leqq L M$. For $r_{0}=5$ we may estimate $4 L M_{1}+r_{0} M_{1}<6 L M_{1}$, and the condition is satisfied if $6 L M_{1} \leqq L M$, or $6 M_{1} \leqq M$. Assuming this, and assuming also that $X_{1}$ is contained in $\Omega_{k}$, we obtain

$$
X \subset \Omega_{k}(X) \subset \cdots \subset \Omega_{1}(X) \subset X_{0}, X_{0}^{\sim r_{0}} \subset X_{1} \subset \Omega_{k}, \quad X_{0}, X_{1} \in \mathscr{D}_{k} .
$$

The idea how to construct a localization expansion of the function (1.1) which we follow here is to replace at first the function $\psi_{k}^{(j)}$ by a local function with a generating set contained in some neighborhood of $X$, and then to introduce the parameters $s$ into the function which is composed with this local one. We use the generating set $\mathbb{B}_{k}(X)$ and the corresponding local function $\phi\left(\mathbb{B}_{k}(X)\right)$. We have

$$
\begin{gathered}
\phi\left(\mathbb{B}_{k} ; \psi\right)=\phi\left(\mathbb{B}_{k}(X) ; \psi\left(\mathbb{B}_{k}(X), \mathbb{B}_{k} ; \psi\right)\right), \\
\phi\left(\mathbb{B}_{k+1} ; \theta\right)=\phi\left(\mathbb{B}_{k}(X) ; \psi\left(\mathbb{B}_{k}(X), \mathbb{B}_{k+1} ; \theta\right)\right) .
\end{gathered}
$$


We take the generating set corresponding to the sequence

$$
\left\{\Omega_{1}, \ldots, \Omega_{j}, \Omega_{j+1}(X), \ldots, \Omega_{k}(X)\right\}
$$

and we denote it by $\mathbb{B}_{j} \cup \mathbb{B}_{k}^{(j)}(X)$. We have

$$
\psi\left(\mathbb{B}_{j}, \mathbb{B}_{k} ; \psi\right)=\psi\left(\mathbb{B}_{j}, \mathbb{B}_{j} \cup \mathbb{B}_{k}^{(j)}(X) ; \psi\left(\mathbb{B}_{j} \cup \mathbb{B}_{k}^{(j)}(X), \mathbb{B}_{k} ; \psi\right)\right)
$$

Consider the function $\psi\left(\mathbb{B}_{j}, \mathbb{B}_{j} \cup \mathbb{B}_{k}^{(j)}(X) ; \psi^{\prime \prime}\right)$. By (4.21) [3] we have

$$
\begin{gathered}
\psi\left(\mathbb{B}_{j}, \mathbb{B}_{j} \cup \mathbb{B}_{k}^{(j)}(X) ; \psi^{\prime \prime}\right)=Q_{j} \phi\left(\mathbb{B}_{j} \cup \mathbb{B}_{k}^{(j)}(X) ; \psi^{\prime \prime}\right)+\frac{a_{p}}{a_{j}}\left(L^{j} L^{-p}\right)^{2} \\
\times Q_{p-j}^{*}\left(\psi_{p}^{\prime \prime}-Q_{p} \phi\left(\mathbb{B}_{j} \cup \mathbb{B}_{k}^{(j)}(X) ; \psi^{\prime \prime}\right)\right) \text { on } B^{p-j}\left(\Lambda_{p}(X)\right), p>j \\
\psi\left(\mathbb{B}_{j}, \mathbb{B}_{j} \cup \mathbb{B}_{k}^{(j)}(X) ; \psi^{\prime \prime}\right)=\psi^{\prime \prime} \quad \text { on } \bigcup_{n=0}^{j} \Lambda_{n} \cup\left(\Omega_{j} \backslash \Omega_{j+1}(X)\right)^{(j)}
\end{gathered}
$$

The function $\phi\left(\mathbb{B}_{j} \cup \mathbb{B}_{k}^{(j)}(X) ; \psi^{\prime \prime}\right)$ can be written in the form

$$
\phi\left(\mathbb{B}_{j} \cup \mathbb{B}_{k}^{(j)}(X) ; \psi^{\prime \prime}\right)=\phi\left(\mathbb{B}_{k}(X) ; \psi\left(\mathbb{B}_{k}(X), \mathbb{B}_{j} \cup \mathbb{B}_{k}^{(j)}(X) ; \psi^{\prime \prime}\right)\right),
$$

and we have

$$
\psi\left(\mathbb{B}_{k}(X), \mathbb{B}_{j} \cup \mathbb{B}_{k}^{(j)}(X) ; \psi^{\prime \prime}\right)=\psi^{\prime \prime} \quad \text { on } \bigcup_{p=j}^{k} \Lambda_{p}(X)
$$

From the above formulas we get

$$
\begin{aligned}
\psi( & \left.\mathbb{B}_{j}, \mathbb{B}_{j} \cup \mathbb{B}_{k}^{(j)}(X) ; \psi^{\prime \prime}\right) \\
= & Q_{j} \phi\left(\mathbb{B}_{k}(X) ; \psi\left(\mathbb{B}_{k}(X), \mathbb{B}_{j} \cup \mathbb{B}_{k}^{(j)}(X) ; \psi^{\prime \prime}\right)\right)+\frac{a_{p}}{a_{j}}\left(L^{j} L^{-p}\right)^{2} Q_{p-j}^{*}\left(\psi \left(\mathbb{B}_{k}(X),\right.\right. \\
& \left.\left.\mathbb{B}_{j} \cup \mathbb{B}_{k}^{(j)}(X) ; \psi^{\prime \prime}\right)-Q_{p} \phi\left(\mathbb{B}_{k}(X) ; \psi\left(\mathbb{B}_{k}(X), \mathbb{B}_{j} \cup \mathbb{B}_{k}^{(j)}(X) ; \psi^{\prime \prime}\right)\right)\right) \\
= & \psi\left(\mathbb{B}_{j}(X), \mathbb{B}_{k}(X) ; \psi\left(\mathbb{B}_{k}(X), \mathbb{B}_{j} \cup \mathbb{B}_{k}^{(j)}(X) ; \psi^{\prime \prime}\right)\right) \text { on } B^{p-j}\left(\Lambda_{p}(X)\right), \\
& p \geqq j,
\end{aligned}
$$

again using the formula (4.21) [3]. Combining (4.10), (4.14) we obtain

$$
\begin{aligned}
& \psi\left(\mathbb{B}_{j}, \mathbb{B}_{k} ; \psi\right) \\
& \quad=\psi\left(\mathbb{B}_{j}(X), \mathbb{B}_{k}(X) ; \psi\left(\mathbb{B}_{k}(X), \mathbb{B}_{j} \cup \mathbb{B}_{k}^{(j)}(X) ; \psi\left(\mathbb{B}_{j} \cup \mathbb{B}_{k}^{(j)}(X), \mathbb{B}_{k} ; \psi\right)\right)\right) \\
& =\psi\left(\mathbb{B}_{j}(X), \mathbb{B}_{k}(X) ; \psi\left(\mathbb{B}_{k}(X), \mathbb{B}_{k} ; \psi\right)\right) \text { on } \Omega_{j}(X)^{(j)} \text {, hence on } X \cap T^{(j)} .
\end{aligned}
$$


This yields finally the identity

$$
\begin{aligned}
\psi & \left(\mathbb{B}_{j}, \mathbb{B}_{k} ; \psi\left(\mathbb{B}_{k}, \mathbb{B}_{k+1} ; \theta\right)+\psi^{\prime}\right) \\
= & \psi\left(\mathbb{B}_{j}(X), \mathbb{B}_{k}(X) ; \psi\left(\mathbb{B}_{k}(X), \mathbb{B}_{k} ; \psi\left(\mathbb{B}_{k}, \mathbb{B}_{k+1} ; \theta\right)+\psi^{\prime}\right)\right) \\
= & \psi\left(\mathbb{B}_{j}(X), \mathbb{B}_{k}(X) ; \psi\left(\mathbb{B}_{k}(X), \mathbb{B}_{k+1} ; \theta\right)+\delta \psi\left(\mathbb{B}_{k}(X), \mathbb{B}_{k} ; \psi^{\prime}\right)\right) \\
& \quad \text { on } \Omega_{j}(X)^{(j)}, \text { hence on } X \cap T^{(j)} .
\end{aligned}
$$

In the three identities above we have taken $\mathbb{B}_{j}(X)$ defined by (4.4)-(4.6) with $j$ instead of $k$. We obtain a sequence of different, smaller domains, in particular $\Omega_{j}^{\prime}=\Omega_{j}(X)=X$ by (4.4). The external function $\psi\left(\mathbb{B}_{j}(X), \mathbb{B}_{k}(X)\right)$ on the righthand side of (4.16), restricted to $X \cap T^{(j)}$, is localized in a small neighborhood of $\Omega_{1}(X)$ (we add one layer of points to this domain), hence in the domain $X_{0}$. Furthermore, regularity properties of this function on $X \cap T^{(j)}$ are basically the same as of the function $\psi\left(\mathbb{B}_{j}, \mathbb{B}_{k}\right)$. This will be analyzed in detail in the future.

Because of these properties we use the representation (4.16) as a basis of our expansion method. We need to introduce the localization parameters only into the internal functions on the right-hand side of (4.16). We discuss it now. We take the localization parameters determined by the generating set $\mathbb{B}_{k}$, or by the sequence of domains $\left\{\Omega_{1}, \Omega_{2}, \ldots, \Omega_{k}\right\}$, which are considered as localization domains with large cubes determined by $M_{1}$. We do not take into account the domain $\Omega_{k+1}$. The parameters $s$ are introduced into the function $\psi\left(\mathbb{B}_{k}(X), \mathbb{B}_{k+1} ; \theta\right)$ in the way discussed before. This function is represented by the formula (4.21) [3] in terms of the function $\phi\left(\mathbb{B}_{k+1} ; \theta\right)$, and we extend the last one to the function $\phi\left(\mathbb{B}_{k+1} ; \theta, s\right)$ determined by the geometric setting constructed for $\mathbb{B}_{k}$, although on cubes intersecting $\Omega_{k+1}$ we take operators corresponding to $k+1^{\text {st }}$ renormalization step. Thus we obtain the extension $\psi\left(\mathbb{B}_{k}(X), \mathbb{B}_{k+1} ; \theta, s\right)$ whose properties are summarized partially in Proposition 2.3. Similarly the function $\delta \psi\left(\mathbb{B}_{k}(X), \mathbb{B}_{k} ; \psi^{\prime}\right)$ is defined by the formula

$$
\begin{aligned}
\delta \psi\left(\mathbb{B}_{k}(X), \mathbb{B}_{k} ; \psi^{\prime}\right)= & Q\left(\mathbb{B}_{k}(X)\right) \delta \phi\left(\mathbb{B}_{k} ; \psi^{\prime}\right)+\frac{1}{a\left(\mathbb{B}_{k}(X)\right)} Q^{*}\left(\mathbb{B}_{k}, \mathbb{B}_{k}(X)\right) a\left(\mathbb{B}_{k}\right) \\
& \times\left(\psi^{\prime}-Q\left(\mathbb{B}_{k}\right) \delta \phi\left(\mathbb{B}_{k} ; \psi^{\prime}\right)\right),
\end{aligned}
$$

where $\delta \phi\left(\mathbb{B}_{k} ; \psi^{\prime}\right)$ is the solution of the system of Eqs. (3.20) [3] with $\delta \psi=\psi^{\prime}, \phi_{0}=$ $\phi\left(\mathbb{B}_{k+1} ; \theta\right), \alpha_{0}=\alpha\left(\mathbb{B}_{k+1} ; \theta\right), f_{1}=0, \alpha_{1}=0$. This solution is an analytic function of $\psi^{\prime}, \phi_{0}, \alpha_{0}$ on any domain (3.21) [3] satisfying the assumptions of Proposition 3.1 [3] and it satisfies the bounds (3.47) [3] of this proposition. We denote the solution by $\delta \phi\left(\mathbb{B}_{k} ; \psi^{\prime}, \phi_{0}, \alpha_{0}\right)$. We introduce the parameters $s$ into this function as in Proposition 2.1, and we define

$$
\delta \phi\left(\mathbb{B}_{k} ; \psi^{\prime}, s\right)=\delta \phi\left(\mathbb{B}_{k} ; \psi^{\prime}, \phi\left(\mathbb{B}_{k+1} ; \theta, s\right), \alpha\left(\mathbb{B}_{k+1} ; \theta, s\right), s\right) .
$$

Let us make a few remarks on domains and analyticity properties of the above function. By Proposition 2.1 the function $\delta \phi\left(\mathbb{B}_{k} ; \psi^{\prime}, \phi_{0}, \alpha_{0}, s\right)$ is analytic on domains (3.21) [3] with $\delta_{1}, \delta_{0}$ satisfying the assumptions of this proposition, in particular $B_{1} \delta_{0} \leqq \frac{1}{8}$. The constant $\delta_{1}$ occurs in the restriction $\left|\psi^{\prime}\right|<\delta_{1}$ only, and we may assume that it is sufficiently small. We have to check when $\phi_{0}=\phi\left(\mathbb{B}_{k+1} ; \theta, s\right), \alpha_{0}=$ $\alpha\left(\mathbb{B}_{k+1} ; \theta, s\right)$ belong to a domain (3.21) [3], or (3.2), with $\delta_{0}$ satisfying the above condition. We consider these functions on a space $\tilde{\Psi}^{c}\left(\mathbb{B}_{k+1} ; \delta, \varepsilon\right)$ with $\delta, \varepsilon$ satisfying 
the assumptions of Proposition 2.2. Then they satisfy the bounds (1.15) [3] with $K_{4}$ instead of $K_{1}$, and these yield immediately the last three bounds in (3.2) with $\delta_{0}=$ $K_{4} \delta$. To bound $\phi_{0}^{2}-1$ we write $\phi_{0}^{2}-1=\left(\phi_{0}-\theta\right)^{2}+2\left(\phi_{0}-\theta\right) \cdot \theta+\left(\theta^{2}-1\right)$, and from the first bound in (1.15) [3] and the definition of the space $\tilde{\Psi}^{c}\left(\mathbb{B}_{k+1} ; \delta, \varepsilon\right)$ we get

$$
\left|\phi_{0}^{2}-1\right| \leqq\left|\phi_{0}-\theta\right|^{2}+2\left|\phi_{0}-\theta\right||\theta|+\left|\theta^{2}-1\right|<K_{4}^{2} \delta^{2}+2 K_{4} \delta \frac{3}{2}+2 \delta<4 K_{4} \delta
$$

for $\delta$ sufficiently small. Similarly we get

$$
|| \phi_{0}|-1| \leqq\left|\phi_{0}-\theta\right|+|| \theta|-1|<K_{4} \delta+\varepsilon+2 \delta<\frac{1}{2}
$$

for $\delta, \varepsilon$ sufficiently small. Thus the two configurations belong to (3.2) with $\delta_{0}=$ $4 K_{4} \delta$, and we assume that $\delta$ is so small that $4 B_{1} K_{4} \delta \leqq \frac{1}{8}$. With these assumptions the function (4.18) is an analytic function of $(\theta, h), \psi^{\prime}, s$ defined on corresponding domains

$$
\tilde{\Psi}^{c}\left(\mathbb{B}_{k+1} ; \delta, \varepsilon\right) \times\left\{\psi^{\prime}:\left|\psi^{\prime}\right|<\delta_{1}\right\} \times\left\{s:|s|<e^{\kappa_{1}}\right\} .
$$

It satisfies the bounds (2.54) of Proposition 2.1. Substituting it into the expression on the right-hand side of (4.17) we obtain an analytic extension $\delta \psi\left(\mathbb{B}_{k}(X), \mathbb{B}_{k} ; \psi^{\prime}, s\right)$ of the function (4.17) defined on the same domains (4.19). From the representation (4.17) and the first bound in (2.54) we obtain the bound

$$
\left|\delta \psi\left(\mathbb{B}_{k}(X), \mathbb{B}_{k} ; \psi^{\prime}, s\right)\right|<\left(2 K_{3}+1\right) \delta_{1} .
$$

The function $\psi\left(\mathbb{B}_{k}(X), \mathbb{B}_{k+1} ; \theta, s\right)$ has properties described in Proposition 2.3, in particular it is defined on the domains (4.19) and it has values in the space $\tilde{\Psi}^{c}\left(\mathbb{B}_{k}(X) ; 2(d+2) K_{4} \delta, \varepsilon\right)$. From all the above statements it follows that we have constructed an analytic extension

$$
\psi\left(\mathbb{B}_{k}(X), \mathbb{B}_{k+1} ; \theta, s\right)+\delta \psi\left(\mathbb{B}_{k}(X), \mathbb{B}_{k} ; \psi^{\prime}, s\right)
$$

of the internal function on the right-hand side of (4.16), defined on the domains (4.19) and having values in the space $\tilde{\Psi}^{c}\left(\mathbb{B}_{k}(X) ; 2(d+2) K_{4} \delta+4\left(2 K_{3}+1\right) \delta_{1}, \varepsilon\right)$. The last condition follows from (4.20) by considering the second term in (4.21) as a part of the complex configuration in the definition (1.12) [3] of this space. Assuming again that $\delta, \varepsilon, \delta_{1}$ are sufficiently small, so that $2(d+2) K_{4} \delta+4\left(2 K_{3}+\right.$ 1) $\delta_{1}, \varepsilon$ satisfy the assumptions of Proposition 4.1 [3], we substitute the function (4.21) into the external function on the right-hand side of (4.16), and we obtain an analytic extension of the function (4.16) in the localization parameters $s$. By Proposition 4.1 [3] it belongs to the space $\tilde{\Psi}^{c}\left(\mathbb{B}_{j}(X) ; \delta_{2}, \varepsilon\right)$, where

$$
\delta_{2}=2(d+2) K_{1} \cdot\left(2(d+2) K_{4} \delta+4\left(2 K_{3}+1\right) \delta_{1}\right) .
$$

Let us recall now that in the function (1.1) we have $\psi^{\prime}=\beta_{k}^{-\frac{1}{2}} C^{(k) \frac{1}{2}} \psi$, and we have to localize this expression also. We have constructed the analytic extension $C^{(k) \frac{1}{2}}\left(\phi_{0}, \alpha_{0} ; s\right)$ in Proposition 3.1, and similarly to (4.18) we define

$$
C^{(k) \frac{1}{2}}(s)=C^{(k) \frac{1}{2}}\left(\phi_{k+1}(s), \alpha_{k+1}(s) ; s\right) .
$$


It is an analytic function on the part of the domain (4.19) without the second factor, and it satisfies the bound (3.24). The variable $\psi$, which is a "fluctuation" variable connected with the $k+1^{\text {st }}$ renormalization transformation, and which will be discussed in the next paper, satisfies the bound $|\psi|<p_{1}\left(\beta_{k}\right)=A_{1}\left(\log \beta_{k}\right)^{p_{1}}$, where $p_{1}$ is an even integer smaller than $p_{0}$, hence

$$
\left|\psi^{\prime}\right|<\beta_{k}^{-\frac{1}{2}} B_{5} p_{1}\left(\beta_{k}\right) \leqq B_{5} C_{\alpha_{1}} \beta_{k}^{-\frac{1}{2}+\alpha_{1}}=\delta_{1} .
$$

Obviously $\delta_{1}$ can be arbitrarily small if $\beta_{k}$ is large enough. In the remaining formulas and estimates we will use for simplicity the variable $\psi^{\prime}$, remembering that

$$
\psi^{\prime}=\beta_{k}^{-\frac{1}{2}} C^{(k) \frac{1}{2}}(s) \psi .
$$

We will come back to a discussion of (4.23) in another paper, where we will construct and discuss all remaining localization expansions.

Thus the extended in $s$ function inside (1.1) is analytic on quite large domains determined by some absolute constants. Unfortunately these analyticity properties do not fit well to the inductive hypotheses, as was explained already in [1], and as should be clear from the above discussion. In particular the domains used do not behave well under the compositions of various minimizing functions. Because of this we have to use spaces of the type $\Xi_{j}^{c}(\sigma, \varepsilon)$ introduced in [1], but localized properly. Nevertheless we need also almost all results of the above analysis done for the domains (4.19).

We formulate now definitions of such generalized spaces. They are simple modifications of the definitions $(2.15),(2.16)$ and $(2.20),(2.21)$ in [1], but because of their importance we write here their complete formulations. Take a generating set $\mathbb{B}_{j}$, and a function $\sigma\left(\mathbb{B}_{j}\right)$ defined on $\Omega_{0}$, having positive values in the interval $\left.] 0,1\right]$, and satisfying the condition that it is constant on corresponding blocks determined by points of $\mathbb{B}_{j}$, i.e., constant on $n$-blocks $\Delta_{n}(y)$ for $y \in \Lambda_{n}, n \leqq j$. We take $\Omega_{0}$ as some "small" neighbourhood of $\Omega_{1}$, for example $\Omega_{0}=\Omega_{1}^{\sim}$, or even a smaller neighbourhood obtained by adding a layer of 1-blocks to $\Omega_{1}$. We introduce the following spaces of configurations $\phi$ defined on $\Omega_{0}$ and having values in $\mathbb{R}^{N}$, and vectors $h \in \mathbb{R}^{N}$

$$
\begin{aligned}
& \Phi\left(\mathbb{B}_{j} ; \sigma\left(\mathbb{B}_{j}\right), \varepsilon ; \lambda, v\right)=\left\{(\phi, h):\left|\partial^{\xi} \phi(x)\right|<\sigma\left(\mathbb{B}_{j} ; x\right)\left(L^{n} \xi\right)^{-1} \varepsilon,\right. \\
& \left|\Delta^{\xi} \phi(x)\right|,|\alpha(x)|<\sigma^{2}\left(\mathbb{B}_{j} ; x\right)\left(L^{n} \xi\right)^{-2} \varepsilon,|\phi(x)-h|<v^{-\frac{1}{2}} \sigma\left(\mathbb{B}_{j} ; x\right)\left(L^{n} \xi\right)^{-1} \varepsilon \\
& \text { on } \Delta_{n}(y), y \in \Lambda_{n}, \text { or simply on } B_{n}\left(\Lambda_{n}\right)=\Omega_{n} \backslash \Omega_{n+1}, n \leqq j, \\
& \left.\left|h^{2}-1\right|<v^{-1} \inf _{n, x \in \Omega_{n} \backslash \Omega_{n+1}} \sigma^{2}\left(\mathbb{B}_{j} ; x\right)\left(L^{n} \xi\right)^{-2} \varepsilon^{2}\right\}, \\
& \alpha=\frac{\lambda}{2}\left(\phi^{2}-1\right),
\end{aligned}
$$

where $\varepsilon, \lambda, v$ are positive numbers. We extend these spaces to complex spaces of configurations $\left(\phi+\phi^{\prime}, h+h\right)$, where $(\phi, h)$ is as above, $\phi^{\prime}$ is defined on $\Omega_{0}$ and has values in $\mathbb{C}^{N}, h^{\prime}$ is defined on $\mathbb{B}_{j}$ and has values in $\mathbb{C}^{N}$. We take

$$
\begin{aligned}
& \Phi^{c}\left(\mathbb{B}_{j} ; \sigma\left(\mathbb{B}_{j}\right), \varepsilon ; \lambda, v\right)=\left\{\left(\phi+\phi^{\prime}, h+h^{\prime}\right):(\phi, h) \in \Phi\left(\mathbb{B}_{j} ; \sigma\left(\mathbb{B}_{j}\right), \varepsilon ; \lambda, \nu\right),\right. \\
& \left|\phi^{\prime}\right|<\varepsilon,\left|\partial^{\xi} \phi^{\prime}(x)\right|<\sigma\left(\mathbb{B}_{j} ; x\right)\left(L^{n} \xi\right)^{-1} \varepsilon,\left|\Delta^{\xi} \phi^{\prime}(x)\right|,|\delta \alpha(x)|
\end{aligned}
$$




$$
\begin{aligned}
& <\sigma^{2}\left(\mathbb{B}_{j} ; x\right)\left(L^{n} \xi\right)^{-2} \varepsilon,\left|h^{\prime}(y)\right|<v^{-1} \sigma^{2}\left(\mathbb{B}_{j} ; y\right)\left(L^{n} \xi\right)^{-2} \varepsilon, \\
& \left.\left|\phi(x) \cdot h^{\prime}(y)\right|<v^{-1} \sigma^{2}\left(\mathbb{B}_{j} ; x\right)\left(L^{n} \xi\right)^{-2} \varepsilon^{2} \text { on } \Delta_{n}(y), y \in \Lambda_{n}, n \leqq j\right\} \\
& \delta \alpha=\frac{\lambda}{2}\left(\left(\phi+\phi^{\prime}\right)^{2}-\phi^{2}\right)=\frac{\lambda}{2}\left(2 \phi \cdot \phi^{\prime}+\phi^{\prime 2}\right) .
\end{aligned}
$$

The above spaces are quite natural in many constructions, in particular Propositions 1.1 [3], 3.1 [3], 2.1, 2.2 can be formulated in terms of these spaces, with $\sigma\left(\mathbb{B}_{j}\right)=1$. Of fundamental importance are spaces of configurations defined on $\mathbb{B}_{j}$. For such configurations $\psi$ with values in $\mathbb{R}^{N}$, and vectors $h \in \mathbb{R}^{N}$, we introduce spaces

$$
\begin{aligned}
& \Xi_{j}\left(\mathbb{B}_{j} ; \sigma\left(\mathbb{B}_{j}\right), \varepsilon\right)=\left\{(\psi, h):\left(\phi_{j}\left(\mathbb{B}_{j} ; \psi, h\right), h\right) \in \Phi\left(\mathbb{B}_{j} ; \sigma\left(\mathbb{B}_{j}\right), \varepsilon ; \lambda_{j}, v_{j}\right),\right. \\
& \left.\left|\psi(y)-\left(Q\left(\mathbb{B}_{j}\right) \phi_{j}\left(\mathbb{B}_{j} ; \psi, h\right)\right)(y)\right|<\sigma^{2}\left(\mathbb{B}_{j} ; y\right) \varepsilon \quad \text { for } y \in \Lambda_{n}, n \leqq j\right\},
\end{aligned}
$$

where $\phi_{j}\left(\mathbb{B}_{j}\right)$ is the minimizing function determined by $\mathbb{B}_{j}$ and by coefficients $a_{j}, \lambda_{j}, v_{j}$. Finally, for complex configurations $\left(\psi+\psi^{\prime}, h+h^{\prime}\right),(\psi, h)$ as above, $\psi^{\prime}, h^{\prime}$ defined on $\mathbb{B}_{j}$ and having values in $\mathbb{C}^{N}$, we define

$$
\begin{aligned}
& \Xi_{j}^{c}\left(\mathbb{B}_{j} ; \sigma\left(\mathbb{B}_{j}\right), \varepsilon\right)=\left\{\left(\psi+\psi^{\prime}, h+h^{\prime}\right):(\psi, h) \in \Xi_{j}\left(\mathbb{B}_{j} ; \sigma\left(\mathbb{B}_{j}\right), \varepsilon\right),\right. \\
& \left(\phi_{j}\left(\mathbb{B}_{j} ; \psi+\psi^{\prime}, h+h^{\prime}\right), h+h^{\prime}\right)=\left(\phi_{j}\left(\mathbb{B}_{j} ; \psi, h\right)+\delta \phi_{j}\left(\mathbb{B}_{j} ; \psi^{\prime}, h^{\prime}\right), h+h^{\prime}\right) \\
& \in \Phi^{c}\left(\mathbb{B}_{j} ; \sigma\left(\mathbb{B}_{j}\right), \varepsilon ; \lambda_{j}, v_{j}\right),\left|\psi^{\prime}(y)-\left(Q\left(\mathbb{B}_{j}\right) \delta \phi_{j}\left(\mathbb{B}_{j} ; \psi^{\prime}, h^{\prime}\right)\right)(y)\right| \\
& \left.<\sigma^{2}\left(\mathbb{B}_{j} ; y\right) \varepsilon \quad \text { for } y \in \Lambda_{n}, n \leqq j\right\} .
\end{aligned}
$$

One of the most important facts relating these spaces to functions $\psi\left(\mathbb{B}_{j}, \mathbb{B}_{k}^{\prime}\right)$ is formulated in the following lemma, a generalization of Lemma 2.1 [1].

Lemma 4.1. For $\varepsilon$ positive and small enough, and $\mathbb{B}_{j} \prec \mathbb{B}_{k}^{\prime}$ (see the definition (4.1) [3]) we have

$$
\psi\left(\mathbb{B}_{j}, \mathbb{B}_{k}^{\prime}\right): \Xi_{k}^{c}\left(\mathbb{B}_{k}^{\prime} ; \sigma\left(\mathbb{B}_{k}^{\prime}\right), \varepsilon\right) \rightarrow \Xi_{j}^{c}\left(\mathbb{B}_{j} ; \sigma\left(\mathbb{B}_{j}, \mathbb{B}_{k}^{\prime}\right) \sigma\left(\mathbb{B}_{k}^{\prime}\right), \varepsilon\right),
$$

where $\sigma\left(\mathbb{B}_{j}, \mathbb{B}_{k}^{\prime}\right)$ is a function defined on $\Omega_{0}$ by the formula

$$
\sigma\left(\mathbb{B}_{j}, \mathbb{B}_{k}^{\prime} ; x\right)=L^{n} L^{-p} \quad \text { for } x \in\left(\Omega_{n} \backslash \Omega_{n+1}\right) \cap\left(\Omega_{p}^{\prime} \backslash \Omega_{p+1}^{\prime}\right) .
$$

Notice that the intersection in (4.30) is empty if $n>p$, by the assumption $\mathbb{B}_{j} \prec \mathbb{B}_{k}^{\prime}$, hence $n \leqq p$ and $\sigma\left(\mathbb{B}_{j}, \mathbb{B}_{k}^{\prime}\right) \leqq 1$. The above lemma follows immediately from the composition formula (4.21) [3] by inspecting the definitions of the spaces involved, in particular corresponding weights on the intersections in (4.30). We could introduce also generalizations of other spaces considered in [1], but in this paper we will use only spaces $\Psi_{j}^{c}(X ; \sigma, \varepsilon)$, which are defined by the conditions (3.13), (3.14) [1] restricted to a localization domain $X$, e.g. $X \in \mathscr{D}_{j}$.

We modify also slightly the inductive assumption (H.5) on the analyticity domains of the localized functions in the expansion (H.5). For a localization domain $X \in \mathscr{D}_{j}$ we construct the minimal generating set $\mathbb{B}_{j}(X)$ (described in (4.4)(4.6)), and we assume that the function $\mathscr{E}^{(j)}\left(y, X ; \psi_{j}, v_{j} h\right)$ has an analytic extension onto the space $\Xi_{j}^{c}\left(\mathbb{B}_{j}(X) ; 1, \varepsilon_{j}\right)$ satisfying the bound and the invariance property in (H.5). This space is larger than the space $\Xi_{j}^{c}\left(1, \varepsilon_{j}\right)$, so the above assumption 
is stronger than the inductive assumption (H.5) in [1]. It implies that a localized function of the expansion (3.127) in [1], corresponding to a domain $X \in \mathscr{D}_{j}$, has an analytic extension onto one of the two spaces: $\Xi_{j}^{c}\left(\mathbb{B}_{j}(X) ; 1, \varepsilon_{j}\right)$, or the subspace of $\Psi_{j}^{c}\left(X ; C_{0} L^{j} \eta, \varepsilon_{k}\right)$ given by the configurations satisfying the additional condition (3.107) [1], and it satisfies the bound (3.128) [1]. We write it more generally as

$$
\left|\mathscr{E}\left(X ; \psi_{j}\right)\right|<E \exp \left(-(\kappa-1) d_{j}(X)\right),
$$

where $E$ is a positive constant, which in applications will be one of the constants in various assumptions and bounds in [1].

Let us consider now the function

$$
\mathscr{E}\left(X ; \psi\left(\mathbb{B}_{j}(X), \mathbb{B}_{k}(X) ; \psi\left(\mathbb{B}_{k}(X), \mathbb{B}_{k+1} ; \theta, s\right)+\delta \psi\left(\mathbb{B}_{k}(X), \mathbb{B}_{k} ; \psi^{\prime}, s\right)\right)\right) .
$$

For $s=1$ and $\psi^{\prime}$ given by (4.24) it is equal to the function (1.1). We would like to prove that it can be analytically extended onto a space

$$
\Xi_{k+1}^{c}\left(\mathbb{B}_{k+1} ; 1, \varepsilon^{\prime}\right) \times\left\{\psi^{\prime}:\left|\psi^{\prime}\right|<\delta_{1}\right\} \times\left\{s:|s|<e^{\kappa_{1}}\right\}
$$

for $\varepsilon^{\prime}<\varepsilon_{k}$, e.g. $\varepsilon^{\prime}=\varepsilon_{k+1}$, and for $\delta_{1}$ sufficiently small. Actually we are interested in a situation where the function depends on the variables restricted to some domain $Y$ containing $X_{1}$, and then we can take $\mathbb{B}_{k+1}$ as equal to $\mathbb{B}_{k+1}(Y)$, so $\mathbb{B}_{k+1}$ in (4.33) has a more general meaning. We will discuss it again later on. To prove the above statement we have to prove that the function inside (4.32) transforms the space (4.33) into the two spaces written above as the two possible analyticity domains. At first we write

$$
\begin{aligned}
& \psi\left(\mathbb{B}_{k}(X), \mathbb{B}_{k+1} ; \theta, s\right)+\delta \psi\left(\mathbb{B}_{k}(X), \mathbb{B}_{k} ; \psi^{\prime}, s\right) \\
& \quad=\psi\left(\mathbb{B}_{k}(X), \mathbb{B}_{k+1} ; \theta\right)+\left[\Delta_{s} \psi\left(\mathbb{B}_{k}(X), \mathbb{B}_{k+1} ; \theta, s\right)+\delta \psi\left(\mathbb{B}_{k}(X), \mathbb{B}_{k} ; \psi^{\prime}, s\right)\right] .
\end{aligned}
$$

The idea is that for the first configuration on the right-hand side we know quite precisely spaces to which it belongs, and the configuration in the square bracket should be sufficiently small. We use (4.29) for the first configuration, and we get

$$
\psi\left(\mathbb{B}_{k}(X), \mathbb{B}_{k+1} ; \theta\right) \in \Xi_{k}^{c}\left(\mathbb{B}_{k}(X) ; \sigma\left(\mathbb{B}_{k}(X), \mathbb{B}_{k+1}\right), \varepsilon^{\prime}\right) \subset \Xi_{k}^{c}\left(\mathbb{B}_{k}(X) ; 1, \varepsilon^{\prime}\right) .
$$

To estimate the configuration in the square bracket we notice that the proofs of Lemmas $4.3,4.4$ [3] can be easily interpreted as yielding

$$
\Xi_{k+1}^{c}\left(\mathbb{B}_{k+1} ; 1, \varepsilon^{\prime}\right) \subset \tilde{\Psi}^{c}\left(\mathbb{B}_{k+1} ; 2(d+2) \varepsilon^{\prime}, 2(d+2) \varepsilon^{\prime}\right) .
$$

By Proposition 2.3 the first term in the square bracket is an analytic function on the space (4.33), if $2(d+2) \varepsilon^{\prime} \leqq c_{7}$, and it satisfies the bound (2.60) with $\delta=2(d+2) \varepsilon^{\prime}$. By the analysis between (4.17) and (4.20) the second term is analytic on this space also, if $\varepsilon^{\prime}$ is sufficiently small to satisfy the conditions listed there, and it satisfies the bound (4.20). Thus the expression in the square bracket on the right-hand side of (4.34) is an analytic function on the space (4.33) and it can be bounded by $\delta_{3}=2(d+2) K_{4} \exp \left(-\gamma_{0} M_{1}\right) \varepsilon^{\prime}+\left(2 K_{3}+1\right) \delta_{1}$, if $\varepsilon^{\prime}, \delta_{1}$ are small enough. Let us take the value of the function $\phi\left(\mathbb{B}_{k}(X)\right)$ on the configuration (4.34). It can be written as the sum

$$
\phi\left(\mathbb{B}_{k}(X) ; \psi\left(\mathbb{B}_{k}(X), \mathbb{B}_{k+1} ; \theta\right)\right)+\delta \phi\left(\mathbb{B}_{k}(X) ;[\ldots]\right),
$$


where the function $\delta \phi\left(\mathbb{B}_{k}(X)\right)$ is defined by the configurations $\phi_{0}=\phi\left(\mathbb{B}_{k+1} ; \theta\right)$, $\alpha_{0}=\alpha\left(\mathbb{B}_{k+1} ; \theta\right), f_{1}=0, \alpha_{1}=0$. It satisfies the bounds (3.47) [3] with $\delta_{3}$ instead of $\delta_{1}$. From this and the definition of the spaces $\Xi_{k}^{c}\left(\mathrm{~B}_{k} ; \sigma, \varepsilon\right)$ we conclude that the configuration (4.34) belongs to the space $\Xi_{k}^{c}\left(\mathbb{B}_{k}(X) ; 1, \varepsilon^{\prime}+\left(K_{2}+1\right) \delta_{3}\right)$, or that the function (4.34) is analytic on the space (4.33) and transforms it into the above one. From this and Lemma 5.1 we obtain the following statement: the function inside (4.32) is defined and analytic on the space (4.33) with $\varepsilon^{\prime}, \delta_{1}$ sufficiently small, and it transforms this space into the space

$$
\Xi_{j}^{c}\left(\mathbb{B}_{j}(X) ; \sigma\left(\mathbb{B}_{j}(X), \mathbb{B}_{k}(X)\right), \varepsilon_{k}\right), \quad \text { if } \varepsilon^{\prime}+\left(K_{2}+1\right) \delta_{3} \leqq \varepsilon_{k} .
$$

Let us analyze the last condition in more detail for $\varepsilon^{\prime}=\varepsilon_{k+1}$, where we take $\varepsilon_{j}$ given by the formula $\varepsilon_{j}=\alpha_{0, k} \xi^{\alpha}, \alpha_{0, k}=\alpha_{0}\left(1+\sum_{n=k}^{+\infty} \frac{1}{n^{2}}\right), \alpha=\frac{d-2}{2}-\gamma, 0<\gamma<$ $\gamma_{0}=\min \left\{1, \frac{d-2}{2}\right\}$. The positive constant $\alpha_{0}$ is sufficiently small, in particular it can be chosen in such a way that all the previous conditions on $\varepsilon^{\prime}$ are satisfied. The last condition is satisfied if $2(d+2)\left(K_{2}+1\right) K_{4} \exp \left(-\gamma_{0} M_{1}\right) \varepsilon_{k}+\left(K_{2}+1\right)\left(2 K_{3}+1\right) \delta_{1} \leqq$ $L^{-\alpha} \alpha \varepsilon_{k}$, because $L^{-\alpha} \alpha \varepsilon_{k} \leqq \varepsilon_{k}-\varepsilon_{k+1}$. We assume now that $M_{1}$ satisfies the additional condition $2(d+2)\left(K_{2}+1\right) K_{4} \exp \left(-\gamma_{0} M_{1}\right) \leqq \frac{1}{2} L^{-\alpha} \alpha$, or $8 d L^{\alpha} K_{2} K_{4} \frac{1}{\alpha} \leqq \exp \gamma_{0} M_{1}$. Then the last condition is satisfied if $\left(K_{2}+1\right)\left(2 K_{3}+1\right) \delta_{1} \leqq \frac{1}{2} L^{-\alpha} \alpha \varepsilon_{k}$, or if

$$
8 L^{\alpha} K_{2} K_{3} \frac{1}{\alpha} \leqq \frac{\alpha_{0} \eta^{\alpha}}{\delta_{1}}, \quad 8 d L^{\alpha} K_{2} K_{4} \frac{1}{\alpha} \leqq \exp \gamma_{0} M_{1}
$$

If we take $\delta_{1}$ given by (4.23), and use the asymptotic properties of $\beta_{k}$, then we obtain the bound $\delta_{1}<2 B_{5} C_{\alpha_{1}} \beta^{-\frac{1}{2}+\alpha_{1}} \eta^{\frac{d-2}{2}-\alpha_{1}(d-2)}$, and the first condition above is satisfied if $\beta^{\frac{1}{2}-\alpha_{1}} \geqq 16 L^{\alpha} B_{5} C_{\alpha_{1}} K_{2} K_{3} \frac{1}{\alpha \alpha_{0}} \eta^{\gamma-\alpha_{1}(d-2)}$. Taking $\alpha_{1}$ such that $\alpha_{1}(d-2) \leqq$ $\gamma$ we obtain that the last condition is satisfied if $\beta$ is large enough, more precisely if $\beta^{\frac{1}{2}-\alpha_{1}} \geqq 16 L^{\alpha} B_{5} C_{\alpha_{1}} K_{2} K_{3} \frac{1}{\alpha \alpha_{0}}$. We will come back to these conditions in one of the following papers, where other localization expansions will be discussed. Now we assume that they are satisfied.

For the space in (4.37) we have the inclusions

$$
\begin{aligned}
& \Xi_{j}^{c}\left(\mathbb{B}_{j}(X) ; \sigma\left(\mathbb{B}_{j}(X), \mathbb{B}_{k}(X)\right), \varepsilon_{k}\right) \subset \Xi_{j}^{c}\left(\mathbb{B}_{j}(X) ; 1, \varepsilon_{j}\right), \\
& \Xi_{j}^{c}\left(\mathbb{B}_{j}(X) ; \sigma\left(\mathbb{B}_{j}(X), \mathbb{B}_{k}(X)\right), \varepsilon_{k}\right)\left\lceil_{X} \subset \Psi_{j}^{c}\left(X ; 3 L^{j} \eta, \varepsilon_{k}\right),\right.
\end{aligned}
$$

where the last one follows from the generalization of Lemma 3.1 in [1] discussed before in connection with Lemmas 4.3, 4.4 [3]. Actually we have to be more precise now, because the representation (3.127) [1] with the bounds (3.128) [1] hold on the subspace of the space $\Psi_{j}^{c}\left(C_{0} L^{j} \eta, \varepsilon_{k}\right)$ defined by the additional condition (3.107) [1], that is the condition $\left|\partial^{2} \psi\right|<K_{1} C_{\alpha_{1}}\left(L^{j} \eta\right)^{2-\alpha_{1}} \varepsilon_{k}$, where $0<\alpha_{1} \leqq 1$ and $C_{\alpha_{1}}$ is an absolute constant depending on $\alpha_{1}$ only. Thus instead of the last inclusion above we have to prove that

$$
\begin{aligned}
& \psi\left(\mathbb{B}_{j}(X), \mathbb{B}_{k}(X)\right)\left\lceil X: \Xi^{c}\left(\mathbb{B}_{k}(X) ; 1, \varepsilon_{k}\right)\right. \\
& \quad \rightarrow\left\{(\psi, h):(\psi, h) \in \Psi_{j}^{c}\left(X ; 3 L^{j} \eta, \varepsilon_{k}\right),\left|\partial^{2} \psi\right|<K_{1} C_{\alpha_{1}}\left(L^{j} \eta\right)^{2-\alpha_{1}} \varepsilon_{k}\right\} .
\end{aligned}
$$

The function $\psi\left(\mathbb{B}_{j}(X), \mathbb{B}_{k}(X)\right)$ has the representation (4.21) [3] in terms of the function $\phi\left(\mathbb{B}_{k}(X)\right)$. We use again the inclusion $\Xi^{c}\left(\mathbb{B}_{k}(X) ; 1, \varepsilon_{k}\right) \subset \tilde{\Psi}^{c}\left(\mathbb{B}_{k}(X) ; 2(d+\right.$ 
2) $\left.\varepsilon_{k}, 2(d+2) \varepsilon_{k}\right)$, and from Proposition 1.1 [3] we obtain that $\phi\left(\mathbb{B}_{k}(X)\right)$ satisfies the third inequality in (1.15) [3] with $\delta$ replaced by $2(d+2) \varepsilon_{k}$. In particular on the domain $X$ we have the inequality with the constant $K_{1} C_{\alpha} 2(d+2) \varepsilon_{k}$ on the right-hand side. Notice that other inequalities in (1.15) [3] hold with $K_{1} \delta$ replaced by $\varepsilon_{k}$, by the definition of the space $\Xi^{c}\left(\mathbb{B}_{k}(X) ; 1, \varepsilon_{k}\right)$. From these inequalities and (4.21) [3] we obtain easily that on the domain $X$

$$
\left|\partial^{2} \psi\left(\mathbb{B}_{j}(X), \mathbb{B}_{k}(X)\right)\right|<K_{1} C_{\alpha_{1}}\left(L^{j} \eta\right)^{2-\alpha_{1}} 2(d+2) \varepsilon_{k}+4\left(L^{j} \eta\right)^{2} \varepsilon_{k},
$$

which implies (4.40) with a properly chosen absolute constant $C_{\alpha_{1}}$ depending on $\alpha_{1}, d$ only. From the inclusions (4.39), (4.40), the statement before (4.37) and the assumptions on the function $\mathscr{E}\left(X, \psi_{j}\right)$ we conclude the following lemma.

Lemma 4.2. The function (4.32) is defined and analytic on the space (4.33) with $\varepsilon^{\prime} \leqq \varepsilon_{k+1}$ and $\delta_{1}$ satisfying the condition (4.38). On this space it satisfies the bound (4.31).

After the above preparatory analysis we can finally construct a localization expansion for the function (1.1). We start with the following simple interpolation identity used many times for similar purposes:

$$
\begin{aligned}
\mathscr{E}\left(X ; \psi_{k}^{(j)}\left(\psi^{(k)}(\theta)+\psi^{\prime}\right)\right) \\
= \\
=\sum_{\mathscr{E}}\left(X ; \psi\left(\mathbb{B}_{j}(X), \mathbb{B}_{k}(X) ; \psi\left(\mathbb{B}_{k}(X), \mathbb{B}_{k+1} ; s_{\square} \frac{\partial}{\partial s_{\square}} \mathscr{E}\left(X ; \psi\left(\mathbb{B}_{j}(X), \mathbb{B}_{k}(X) ; \psi\left(\mathbb{B}_{k}(X), \mathbb{B}_{k+1} ; \theta, s\right)\right.\right.\right.\right.\right. \\
\left.\left.\quad+\delta \psi\left(\mathbb{B}_{k}(X), \mathbb{B}_{k} ; \psi^{\prime}, s\right)\right)\right)\left.\right|_{s(\mathscr{C} c)=0} .
\end{aligned}
$$

The summation above is over all subfamilies $\mathscr{C}$ of the family of large cubes (in the scale $M_{1}$ ) determined by the generating set $\mathbb{B}_{k}$ and contained in $X_{1}^{c}$, the complement $\mathscr{C}^{c}$ is with respect to this family. From now on let us assume for simplicity that $X \subset X_{1} \subset \Omega_{k+1}$. Let us consider the domain

$$
Y_{1}=X_{1} \cup \bigcup_{\square \in \mathscr{C}} \square,
$$

by which we mean, as usual, the domain obtained by adding proper lower dimensional walls (i.e. the walls such that all cubes containing them in their boundaries belong to $\mathscr{C}$ ). This domain is generally a union of several components. By the localization property discussed above the function (4.32) for $s$ such that $s=0$ on $\mathscr{C}^{c}$, i.e. $s\left(\mathscr{C}^{c}\right)=0$, depends on $(\theta, h), \psi^{\prime}, s$, restricted to the component of $Y_{1}$ containing the domain $X$, or $X_{1}$. If there are other non-empty components of $Y_{1}$, then the corresponding term on the right-hand side of (4.41) vanishes, because of the derivatives $\frac{\partial}{\partial s_{\square}}$ for $\square$ contained in those other components. Thus we can write the sum as a sum over connected domains $Y_{1}$ of the form (4.42), and the product over $\square \in \mathscr{C}$ can be replaced by a product over $\square \subset Y_{1} \cap X_{1}^{c}$. We obtain an expansion of the form

$$
\mathscr{E}\left(X ; \psi_{k}^{(j)}\left(\psi^{(k)}(\theta)+\psi^{\prime}\right)\right)=\sum_{Y_{1}: X_{1} \subset Y_{1}} \mathscr{E}^{\prime}\left(X, Y_{1} ; \theta, \psi^{\prime}\right)
$$


A term of this expansion is equal to the corresponding term in (4.41) with $\mathscr{C}$ related to $Y_{1}$ by (4.42). It is almost a desired localization expansion, in particular it satisfies the most important localization property: the function $\mathscr{E}^{\prime}\left(X, Y_{1} ; \theta, \psi^{\prime}\right)$ depends on $\theta, \psi^{\prime}$ restricted to the domain $Y_{1}$. Unfortunately one feature is still missing, the domains $Y_{1}$ in the sum are not localization domains. We correct it by doing partial resummations in the above sum.

At first we assign to each domain $Y_{1}$ in (4.43) a localization domain $Y \in \mathscr{D}\left(\mathbb{B}_{k+1}\right)$ containing $Y_{1}$, and in some sense minimal. For a cube $\square_{1} \in \mathscr{C}, \square_{1} \subset$ $\Omega_{j} \backslash \Omega_{j+1}$ (hence a cube of the size $M_{1} L^{j} \eta$ ) we take the cube $\square \in \pi_{j}$ (hence the cube of the size $M L^{j} \eta$ ) containing $\square_{1}$, and we take the domain $\bigcup\left\{\square^{\prime}: \square^{\prime} \in \pi_{j}^{\prime}, \square^{\prime} \supset\right.$ $\left.\square, \square^{\prime} \subset \Omega_{j} \backslash \Omega_{j+1}\right\}$. This family of cubes is non-empty because each component of $\Omega_{j} \backslash \Omega_{j+1}$ belongs to $\mathscr{D}_{j}$. We obtain a domain from $\mathscr{D}_{j}$ containing $\square$, hence $\square_{1}$, and contained in $\square^{\sim L}$ (which is the cube of the size $(2 L+1) M L^{j} \eta$ with $\square$ in its center). Strictly speaking this construction is described for $j \leqq k$, for $j=k+1$ we still have cubes $\square_{1}$ of the size $M_{1}$, but we take the corresponding cubes $\square \in \pi_{k+1}$, i.e., of the size $M L$, the rest of the construction is the same. We take a union of these domains over all $\square_{1} \in \mathscr{C}$, and we obtain a domain, which is a union of connected components denoted $Y^{(1)}, \ldots, Y^{(n)}$. Each component $Y^{(i)} \in \mathscr{D}\left(\mathbb{B}_{k+1}\right)$, and contains at least one component of $\bigcup \mathscr{C}$, hence a cube $\square_{1} \in \mathscr{C}$ touching $X_{1}$ along some $d$-1-dimensional wall. Take one of those cubes and denote the corresponding cube $\square$ by $\square^{(i)}$. Each component $Y^{(i)}$ is a union of large cubes in the scale $M$, more precisely $Y^{(i)} \cap\left(\Omega_{j} \backslash \Omega_{j+1}\right)$ is a union of cubes from $\pi_{j}$. Denote this family of large cubes by $\mathscr{C}^{(i)}$, hence $\bigcup \mathscr{C}^{(i)}=Y^{(i)}$. We define

$$
Y=X_{1} \cup \bigcup_{i=1}^{n} Y^{(i)}
$$

Obviously $Y \in \mathscr{D}\left(\mathbb{B}_{k+1}\right)$, and there may be many domains $Y_{1}$ determining the same domain $Y$. We do a partial resummation of the terms in (4.43) over the domains $Y_{1}$ determining a fixed $Y$, and we define

$$
\mathscr{E}\left(X, Y ; \theta, \psi^{\prime}\right)=\sum_{Y_{1}: Y_{1} \text { determines } Y} \mathscr{E}^{\prime}\left(X, Y_{1} ; \theta, \psi^{\prime}\right) .
$$

Each term in the above sum depends on $\theta, \psi^{\prime}$, or rather $\theta, \psi$, restricted to $Y_{1} \subset Y$, so the sum depends on these variables restricted to $Y$. In order to formulate analyticity properties of the above function we have to define the generating set $\mathbb{B}_{k+1}(Y)$. It has been defined by (4.4)-(4.6) if $Y \subset \Omega_{k+1}$, but generally we have $Y \in \mathscr{D}\left(\mathbb{B}_{k+1}\right)$ and $Y$ may intersect the domains $\Omega_{j} \backslash \Omega_{j+1}$ for $j \leqq k$, so we have to modify this definition properly. Such a modification is rather obvious, we take $\Omega_{k+1}^{\prime}=Y \cap \Omega_{k+1}$, $\Omega_{k}^{\prime}=\Omega_{k+1}^{\prime \sim 5 L} \cup\left(Y \cap \Omega_{k} \cap \Omega_{k+1}^{c}\right)$, where we add layers of $M_{1}$-cubes, $\ldots, \Omega_{n}^{\prime}=\Omega_{n+1}^{\prime \sim 5 L} \cup$ $\left(Y \cap \Omega_{n} \cap \Omega_{n+1}^{c}\right)$, where we add layers of $M_{1} L^{n} \eta$-cubes, ..., and so on. Obviously we have $\Omega_{j}^{\prime} \subset \Omega_{j}$. The sequence $\left\{\Omega_{1}^{\prime}, \Omega_{2}^{\prime}, \ldots, \Omega_{k}^{\prime}, \Omega_{k+1}^{\prime}\right\}$ is determined by the localization domain $Y$, and we denote it as before by $\left\{\Omega_{1}(Y), \Omega_{2}(Y), \ldots, \Omega_{k}(Y), \Omega_{k+1}(Y)\right\}$. The corresponding generating set is denoted by $\mathbb{B}_{k+1}(Y)$, and we have $\mathbb{B}_{k+1}(Y) \prec \mathbb{B}_{k+1}$. Consider a term in the sum (4.45) corresponding to a domain $Y_{1}$. It is defined by the formula in (4.41) in terms of the function (4.32), in which we take $s=0$ on $\mathscr{C}^{c}$. This function is localized completely in $Y_{1}$, as it follows from the constructions of the two previous sections, from the way the localization parameters $s$ have been introduced. This means that if we take a different generating set $\mathbb{B}_{k+1}^{\prime}$, but such 
that $Y_{1} \cap \mathbb{B}_{k+1}^{\prime}=Y_{1} \cap \mathbb{B}_{k+1}$, then the function (4.32) is equal to the corresponding function with $\mathbb{B}_{k+1}^{\prime}$ instead of $\mathbb{B}_{k+1}$, assuming that the parameters $s^{\prime}$ connected with $\mathbb{B}_{k+1}^{\prime}$ are equal to 0 outside $Y_{1}$, and are equal to $s$ inside $Y_{1}$. Obviously we have $Y_{1} \cap \mathbb{B}_{k+1}(Y)=Y_{1} \cap \mathbb{B}_{k+1}$ by the definition of $Y$ and $\mathbb{B}_{k+1}(Y)$, so we may replace $\mathbb{B}_{k+1}$ by $\mathbb{B}_{k+1}(Y)$ in the definitions of all the terms of the sum (4.45). Applying Lemma 4.2 we conclude that the functions (4.32) determining these terms are analytic on the space (4.33) with $\mathbb{B}_{k+1}(Y)$ instead of $\mathbb{B}_{k+1}$. The terms are defined by the expressions in (4.41) involving integrals of derivatives with respect to $s_{\square}$ for $\square \in \mathscr{C}$. We replace the derivatives by the Cauchy formulas for the circles $\left|s_{\square}\right|=e^{\kappa_{1}}$, or arbitrarily close circles, and we obtain formulas for functions $\mathscr{E}^{\prime}\left(X, Y_{1} ; \theta, \psi^{\prime}\right)$, which imply that they are analytic functions defined on the spaces

$$
\Xi_{k+1}^{c}\left(\mathbb{B}_{k+1}(Y) ; 1, \varepsilon^{\prime}\right) \times\left\{\psi^{\prime}:\left|\psi^{\prime}\right|<\delta_{1}\right\}
$$

with $\varepsilon^{\prime}, \delta_{1}$ as discussed previously in connection with Lemma 4.2. On the above spaces they satisfy the bounds

$$
\left|\mathscr{E}^{\prime}\left(X, Y_{1} ; \theta, \psi^{\prime}\right)\right|<E e^{-\left(\kappa_{1}-1\right)|\mathscr{C}|} \exp \left(-(\kappa-1) d_{j}(X)\right)
$$

for $\kappa_{1} \geqq \log \left(1+\frac{1}{\sqrt{e}-1}\right)$, or simply for $\kappa_{1} \geqq 2$. From this and (4.45) we obtain that $\mathscr{E}\left(X, Y ; \theta, \psi^{\prime}\right)$ is also defined and analytic on the spaces (4.46). We would like to estimate this function in terms of exponential factors involving linear sizes of $X, Y$. The relevant linear size of $X$ is $d_{j}(X)$, and for $Y$ we take $\tilde{d}_{k+1}(Y)$ defined by (1.3).

In order to find a relation between $|\mathscr{C}|$ (which is a number of cubes in the family $\mathscr{C}), d_{j}(X)$ and $\tilde{d}_{k+1}(Y)$, we construct a proper tree graph for the domain $Y$. For a domain $Y^{(i)}$ we take a tree graph $\Gamma_{0}^{(i)}$ starting at the center of $\square^{(i)}$ and built of segments connecting centers of nearest neighbor cubes, if they are of the same size. If they have different sizes, then we take a shortest path connecting their centers and contained in their union. We can take this tree graph in such a way that to each cube in $\mathscr{C}^{(i)}$, except the cube $\square^{(l)}$, we can assign one segment, or one path. The scaled length $|\cdot|_{\mathbb{B}_{k+1}}$ of each segment is equal to 1 , and the scaled length of a path can be estimated by $1+\frac{1}{2}(d-1)=\frac{1}{2}(d+1)$. From this we obtain the following simple bound

$$
\left|\Gamma_{0}^{(i)}\right|_{\mathbb{B}_{k+1}}<\frac{1}{2}(d+1)\left(\left|\mathscr{C}^{(i)}\right|-1\right) \leqq \frac{1}{2}(d+1)(2 L+1)^{d}\left|\mathscr{C} \cap Y^{(i)}\right|,
$$

where in the second inequality we have used the fact that a cube in $\mathscr{C}$ may generate $(2 L+1)^{d}$ cubes in $\mathscr{C}^{(i)}$. Now take a tree graph $\Gamma_{0} \subset X$ satisfying the conditions of the definition of $d_{j}(X)$, and such that $\frac{1}{M L^{\prime} \eta}\left|\Gamma_{0}\right|<d_{j}(X)+\varepsilon$, where $\varepsilon$ is an arbitrary positive number, and the length $\left|\Gamma_{0}\right|$ is taken in the $\eta$-scale. This tree graph intersects every cube from the cover $\pi_{j}^{\prime}$ contained in $X$, so it intersects every cube from the cover $\pi_{k+1}^{\prime}$ contained in $X_{1}$. The cube $\square^{(i)}$ touches a cube from $\pi_{k+1}^{\prime}$ contained in $X_{1}$ along a $d-1$-dimensional wall, and the last one intersects $\Gamma_{0}$, so we connect $\Gamma_{0}$ with the center of $\square^{(i)}$ by a shortest path, and we combine this path with the tree graph $\Gamma_{0}^{(i)}$ obtaining this way a tree graph $\Gamma^{(i)}$. The scaled length of the path can be estimated by $d L+\frac{1}{2}$, so we get

$$
\left|\Gamma^{(i)}\right|_{\mathbb{B}_{k+1}}<\frac{1}{2}(d+1)(2 L+1)^{d}\left|\mathscr{C} \cap Y^{(i)}\right|+d L+\frac{1}{2}<d(2 L+1)^{d}\left|\mathscr{C} \cap Y^{(i)}\right| .
$$


We combine now all the graphs and we take the graph $\Gamma=\Gamma_{0} \cup \Gamma^{(1)} \cup \cdots \cup \Gamma^{(n)}$. It is a tree graph, if the paths connecting $\Gamma_{0}$ with the centers of the cubes $\square^{(i)}$ are disjoint, which is always possible to achieve, and it is contained in $Y$. It is also clear that it intersects every cube from the cover $\pi_{k+1}^{\prime}$ contained in $Y \cap \Omega_{k+1}$, and every cube from one of the covers $\pi_{j}^{\prime}$ contained in $Y \cap\left(\Omega_{j} \backslash \Omega_{j+1}\right)$. Thus we have $|\Gamma|_{\mathbb{B}_{k+1}} \geqq \tilde{d}_{k+1}(Y)$. On the other hand $\Gamma_{0} \subset X \subset \Omega_{k+1}$, hence $\left|\Gamma_{0}\right|_{\mathbb{B}_{k+1}}=\frac{1}{M} L^{-1}\left|\Gamma_{0}\right|$, and

$$
\begin{aligned}
|\Gamma|_{\mathbb{B}_{k+1}}= & L^{-1} \frac{1}{M}\left|\Gamma_{0}\right|+\sum_{i=1}^{n}\left|\Gamma^{(i)}\right|_{\mathbb{B}_{k+1}}<L^{-1} L^{j} \eta\left(d_{j}(X)+\varepsilon\right) \\
& +\sum_{i=1}^{n} d(2 L+1)^{d}\left|\mathscr{C} \cap Y^{(i)}\right|=L^{-1} L^{j} \eta\left(d_{j}(X)+\varepsilon\right)+d(2 L+1)^{d}|\mathscr{C}| .
\end{aligned}
$$

Combining the two bounds and taking the limit $\varepsilon \rightarrow 0$ we obtain the inequality

$$
\tilde{d}_{k+1}(Y) \leqq L^{-1} L^{j} \eta d_{j}(X)+d(2 L+1)^{d}|\mathscr{C}|,
$$

which yields the desired relation between the three quantities involved.

Now we would like to estimate the function given by the formula (4.45). A term in the sum is bounded in (4.47). For the exponent of the exponential functions on the right-hand side of this bound we have

$$
\begin{aligned}
\left(\kappa_{1}-1\right)|\mathscr{C}|+(\kappa-1) d_{j}(X) & \\
= & \left(\frac{1}{2} \kappa_{1}-1\right)|\mathscr{C}|+\left(\frac{1}{3} \kappa-1\right) d_{j}(X)+\frac{\kappa_{1}}{2 d(2 L+1)^{d}} d(2 L+1)^{d}|\mathscr{C}| \\
& +\frac{2 L}{3}\left(L^{j} \eta\right)^{-1} \kappa L^{-1} L^{j} \eta d_{j}(X) \geqq \kappa_{2}|\mathscr{C}|+\kappa_{0} d_{j}(X)+2 \kappa \tilde{d}_{k+1}(Y),
\end{aligned}
$$

where we have assumed that $\frac{1}{3} \kappa-1 \geqq \kappa_{0}, \frac{\kappa_{1}}{2 d(2 L+1)^{d}} \geqq 2 \kappa$, or $\kappa_{1} \geqq 4 d(2 L+1)^{d} \kappa$, and we have denoted $\kappa_{2}=\frac{1}{2} \kappa_{1}-1$. To estimate the sum in (4.45) we use the exponential factor $e^{-\kappa_{2}|\mathscr{C}|}$. We start with the following simple inequality:

$$
\sum_{Y_{1}}^{\prime} e^{-\kappa_{2}|\mathscr{C}|} \leqq \prod_{j=0}^{k+1} \sum_{Y_{1} \cap\left(\Omega_{j} \backslash \Omega_{j+1}\right)}^{\prime} e^{-\kappa_{2}\left|\mathscr{C} \cap\left(\Omega_{j} \backslash \Omega_{j+1}\right)\right|}
$$

where the "primes" mean that the sums are restricted to the admissible domains determining a fixed domain $Y$. Using again the fact that a cube in $\mathscr{C} \cap\left(\Omega_{j} \backslash \Omega_{k+1}\right)$ may generate $(2 L+1)^{d}$ cubes from $\pi_{j}$ in $Y \cap X_{1}^{c} \cap\left(\Omega_{j} \backslash \Omega_{j+1}\right)$ we obtain the bound

$$
(2 L+1)^{d}\left|\mathscr{C} \cap\left(\Omega_{j} \backslash \Omega_{j+1}\right)\right| \geqq \frac{1}{M^{d}}\left|Y \cap X_{1}^{c} \cap\left(\Omega_{j} \backslash \Omega_{j+1}\right)\right|_{\xi},
$$

where the volume is taken in the $\xi$-scale, $\xi=L^{-j}$. We take half of the exponent in the exponential function on the right-hand side of the previous inequality and we apply the above bound. The remaining half yields the product $\prod_{\square_{1} \subset Y_{1} \cap X_{1}^{c} \cap\left(\Omega_{j} \backslash \Omega_{j+1}\right)} e^{-\frac{1}{2} \kappa_{2}}$ We can estimate the primed sum on the right-hand side by a sum over all subdomains of $Y \cap X_{1}^{c} \cap\left(\Omega_{j} \backslash \Omega_{j+1}\right)$ which are unions of large cubes in the scale $M_{1}$, and 
the last sum of the above products is equal to $\left(1+e^{-\frac{1}{2} \kappa_{2}}\right)^{\frac{1}{M_{1}^{d}}\left|Y \cap X_{1}^{c} \cap\left(\Omega_{j} \backslash \Omega_{j+1}\right)\right|_{\xi}}$. Thus we can bound the whole primed sum on the right-hand side by

$$
\exp \left[-\left(\frac{1}{2} \kappa_{2}-e^{-\frac{1}{2} \kappa_{2}}(2 L+1)^{d} \frac{M^{d}}{M_{1}^{d}}\right) \frac{1}{(2 L+1)^{d} M^{d}}\left|Y \cap X_{1} \cap\left(\Omega_{j} \backslash \Omega_{j+1}\right)\right|_{\xi}\right] .
$$

The expression in the parenthesis is equal to a value of the function $f(x)=x-$ $e^{-x} A$ at $x=\frac{1}{2} \kappa_{2}$, where $A=(2 L+1)^{d} \frac{M^{d}}{M_{1}^{d}}$. This is an increasing function, because $f^{\prime}(x)=1+e^{-x} A>0$, and $f(\log A)=\log A-1>0$, hence if $\frac{1}{2} \kappa_{2} \geqq \log A$, then $f\left(\frac{1}{2} \kappa_{2}\right)>0$. With this assumption on $\kappa_{2}$ the above exponential can be bounded by 1 , and we obtain that

$$
\sum_{Y_{1}: Y_{1}} e^{-\kappa_{2}|\mathscr{C}|} \leqq 1
$$

The assumption on $\kappa_{2}$ translates into an assumption on $\kappa_{1}$ of the form $\kappa_{1} \geqq$ $4 d \log \left((2 L+1) \frac{M}{M_{1}}\right)+2$. Let us analyze this assumption in more detail. We have only two conditions on the quotient $\frac{M}{M_{1}}$ : that it is a power $L^{m_{0}}$, where $m_{0}$ is a natural number, and that it is $\geqq 14 d$. We can replace the above assumption on $\kappa_{1}$ by the slightly stronger one $\kappa_{1} \geqq 8 d m_{0} \log (2 L+1)$, and taking the smallest number $m_{0}$ we still obtain a weaker condition than the one introduced previously, i.e., $\kappa_{1} \geqq 4 d(2 L+1)^{d} \kappa$. We fix $\kappa_{1}$ satisfying both conditions.

The expansion (4.43), the definition (4.45) and the bounds (4.47), (4.48), (4.49), (4.50) yield the expansion

$$
\mathscr{E}\left(X ; \psi_{k}^{(j)}\left(\psi^{(k)}(\theta)+\psi^{\prime}\right)\right)=\sum_{Y \in \mathscr{D}\left(\mathbb{B}_{k+1}\right): X, \subset Y} \mathscr{E}\left(X, Y ; \theta, \psi^{\prime}\right),
$$

whose terms are analytic functions on the domains (4.46), satisfying the bounds

$$
\left|\mathscr{E}\left(X, Y ; \theta, \psi^{\prime}\right)\right|<E \exp \left(-\kappa_{0} d_{j}(X)-2 \kappa \tilde{d}_{k+1}(Y)\right) .
$$

Let us formulate the most important results on the localization expansion constructed in this section in the following proposition.

Proposition 4.3. Let us consider the function (1.1), where $\mathscr{E}\left(X, \psi_{j}\right)$ is analytic on a complex space containing one of the two spaces $\Xi_{j}^{c}\left(\mathbb{B}_{j}(X) ; 1, \varepsilon_{j}\right), \Psi_{j}^{c}\left(X ; C_{0} L^{j} \eta, \varepsilon_{k}\right)$ with the condition (3.107) [1] and it satisfies the bound (4.31). The function (1.1) has a localization expansion of the form (4.51), whose terms are analytic functions on the spaces (4.46) with $\varepsilon^{\prime}, \delta_{1}$ satisfying the conditions $\varepsilon^{\prime} \leqq \varepsilon_{k+1}$, (4.38). They depend on $\theta, \psi^{\prime}$, or $\theta, \psi$, restricted to $Y$, and they satisfy the bounds (4.52).

The construction of the localization expansion above is quite universal and we will apply it in several other situations. Notice that until now we have obtained only one condition on $\kappa$, namely $\frac{1}{3} \kappa-1 \geqq \kappa_{0}$, or $\kappa \geqq 3 \kappa_{0}+3$. Notice also that we have formulated and proved it under the simplifying assumption $X \subset X_{1} \subset \Omega_{k+1}$. Later we will have to discuss also some simple variations, like $X_{1} \subset \Omega_{k}$. We have also considered the two versions of $\psi^{\prime}$, one as an independent complex variable, and the other given by the formula (4.24). This will be also used in the future for some simple generalizations. 


\section{References}

1. Balaban, T.: A low temperature expansion for classical $N$-vector models I. A renormalization group flow. Commun. Math. Phys. 167, 103-154 (1995)

2. Balaban, T.: (a) Commun. Math. Phys. 89, 571 (1983); (b) Commun. Math. Phys. 96, 223 (1982)

3. Balaban, T.: The variational problems for classical $N$-vector models. Preprint, to be published in Commun. Math. Phys.

Communicated by D. Brydges 